University of Louisville ThinkIR: The University of Louisville's Institutional Repository

Electronic Theses and Dissertations

$12-2015$

\title{
Chemoattractant receptors BLT1 and CXCR3 regulate anti-tumor immunity by facilitating CD $8+$ T cell migration to tumors.
}

Zinal S. Chheda

University of Louisville

Follow this and additional works at: https://ir.library.louisville.edu/etd

Part of the Immunity Commons

\section{Recommended Citation}

Chheda, Zinal S., "Chemoattractant receptors BLT1 and CXCR3 regulate anti-tumor immunity by facilitating CD8+ T cell migration to tumors." (2015). Electronic Theses and Dissertations. Paper 2294.

https://doi.org/10.18297/etd/2294

This Doctoral Dissertation is brought to you for free and open access by ThinkIR: The University of Louisville's Institutional Repository. It has been accepted for inclusion in Electronic Theses and Dissertations by an authorized administrator of ThinkIR: The University of Louisville's Institutional Repository. This title appears here courtesy of the author, who has retained all other copyrights. For more information, please contact thinkir@louisville.edu. 


\title{
CHEMOATTRACTANT RECEPTORS BLT1 AND CXCR3 \\ REGULATE ANTI-TUMOR IMMUNITY BY FACILITATING \\ CD8 $^{+}$T CELL MIGRATION TO TUMORS
}

\author{
By \\ Zinal S. Chheda \\ M.Sc. in Biotechnology, University of Mumbai, 2009. \\ M.S. University of Louisville, 2012.
}

\begin{abstract}
A Dissertation
Submitted to the Faculty of the School of Medicine of the University of Louisville in Partial Fulfillment of the Requirements for the Degree of
\end{abstract}

Doctor of Philosophy

in Microbiology and Immunology

Department of Microbiology and Immunology

University of Louisville

Louisville, Kentucky

December 2015 
Copyright 2015 by Zinal S. Chheda

(C)All rights reserved 



\section{CHEMOATTRACTANT RECEPTORS BLT1 AND CXCR3 \\ REGULATE ANTI-TUMOR IMMUNITY BY FACILITATING \\ CD8 $^{+}$T CELL MIGRATION TO TUMORS \\ By}

Zinal S. Chheda

M.Sc. University of Mumbai, 2009

M.S. University of Louisville, 2012

A Dissertation Approved on

$6^{\text {th }}$ November, 2015

by the following Dissertation Committee

Haribabu Bodduluri, Ph.D. (Dissertation Director)

Haval Shirwan, Ph.D.

Michele Kosiewicz, Ph.D.

Jun Yan, M.D., Ph.D.

Susan Galandiuk, M.D. 


\section{ACKNOWLEDGEMENTS}

I would like to express my heartfelt gratitude towards my mentor Dr. Haribabu Bodduluri for all his constant support, encouragement and patience throughout the five years. I cannot thank you enough for sharing your insight and expertise which has greatly helped in developing my career as a future scientist and a critical thinker.

I would also like to thank my committee members, Dr. Haval Shirwan, Dr. Michelle Kosiewicz, Dr. Jun Yan and Dr. Susan Galandiuk for all your insight, valuable suggestions and scientific discussions throughout these five years. Your unified inputs alongwith positive feedbacks made this dissertation possible.

A special thanks to Dr. Rajesh Sharma for his contributions in this project and for all the scientific discussions, teachings and encouragement at every step of my Ph.D. I thank him for teaching me all the nitty-gritty and the fundamentals of immunology.

I would want to thank Dr. Venkatakrishna Jala for his valuable guidance, support, and technical expertise. Thanks to all the lab members of the Bodduluri Lab, Dr. Shuchismita Satpathy, Dr. Sobha Bodduluri, Dr. BiswaPratim Das, Bindu Hegde and Ruquiah for all their kind support, scientific discussions, motivation

and advice. I thank the present lab manager Rebecca Lindsey. Completing this work was much easier with you in the lab. I would like to thank previous lab 
members, Dr. Steven Mathis and Michelle Smith for their scientific teachings, kind support and great discussions on life. I cherish all the wonderful time spent together with all of you in the lab.

I thank the Department of Microbiology and Immunology, staff and faculty members as well as the students that made it such a pleasure to work and learn here.

The support and friendship of my friends, here in Louisville and in India have been invaluable for which I am extremely thankful. I thank them for their constant support and all the fun times.

Most importantly, none of this would have been possible without the unconditional love, support and encouragement of my parents and my family for which my mere expression of thanks would not suffice. 


\begin{abstract}
CHEMOATTRACTANT RECEPTORS BLT1 AND CXCR3

REGULATE ANTI-TUMOR IMMUNITY BY FACILITATING

CD8 ${ }^{+}$T CELL MIGRATION TO TUMORS
\end{abstract}

By

Zinal Chheda

$6^{\text {th }}$ November, 2015

Presence of increased numbers of $\mathrm{CD}^{+} \mathrm{T}$ cells in the tumors correspond to better overall survival in the patients. Variety of immuno-therapies have shown considerable efficacy in the clinic, however, a multitude of patients remain unresponsive. Most of these immunotherapies rely on effector T cell responses in the tumor. A major obstacle in the success of these immunotherapies is poor recruitment of $\mathrm{CD}^{+} \mathrm{T}$ cells into tumors despite intact effector responses in the periphery. Therefore understanding the mechanisms that regulate CTL infiltration into tumors becomes essential. Previous studies in our laboratory suggested an important role for BLT1 in immune surveillance against tumors by regulating CTL migration in a syngeneic cervical cancer tumor model. In this thesis, we 
investigated the roles of leukotriene $\mathrm{B}_{4}\left(\mathrm{LTB}_{4}\right)$ receptor - BLT1; and CXCR3, the receptor for CXCL9, CXCL10 and CXCL11 in anti-tumor immunity using a syngeneic $\mathrm{B} 16$ melanoma tumor model. $\mathrm{BLT}^{-/}$mice and $\mathrm{CXCR}^{-/}$mice on a C57BL/6 background were used to examine the function of these receptors in tumor progression. Significant acceleration in tumor growth and reduced survival was observed in both $\mathrm{BLT} 1^{-/}$and $\mathrm{CXCR} 3^{-/-}$mice as compared to the WT mice. Analysis of tumor infiltrating leukocytes revealed significant reduction of $\mathrm{CD}^{+} \mathrm{T}$ cells in the tumors of $\mathrm{BLT}^{-/}$and $\mathrm{CXCR}^{-/-}$mice as compared to WT tumors; their frequencies being similar in the periphery (spleen and TdLN). Significant reduction of Granzyme-B and IFNy transcripts were observed in tumors of knockout mice compared to WT mice.

Adoptive transfer of tumor experienced WT but not $\mathrm{BLT}^{-/-}$or $\mathrm{CXCR}^{-/-} \mathrm{CD}^{+} \mathrm{T}$ cells reduced tumor growth significantly in Rag2 ${ }^{-/}$mice, which correlated with reduced infiltration of knockout $\mathrm{CD} 8^{+} \mathrm{T}$ cells into tumors. Co-transfer of WT CD8 ${ }^{+}$ $\mathrm{T}$ cells with either of the knockout $\mathrm{CD}^{+} \mathrm{T}$ cells in tumor bearing $\mathrm{Rag}^{-/-}$mice showed that WT $\mathrm{CD} 8^{+} \mathrm{T}$ cells did not facilitate additional knockout $\mathrm{CD} 8^{+} \mathrm{T}$ cell infiltration to tumors. BLT1/CXCR3 double deficient mice displayed similar tumor kinetics as single knockout mice and showed lack of synergism.

The requirement for BLT1 and CXCR3 in inducing checkpoint blockade mediated anti-tumor response was tested. While anti-PD-1 based vaccine significantly attenuated tumor growth in WT mice, the vaccine completely lost its efficacy in $\mathrm{BLT}^{-/-}, \mathrm{CXCR}^{-/}$or $\mathrm{BLT}^{-/-} \mathrm{CXCR}^{-/-}$mice that correlated with failure of knockout $\mathrm{CD}^{+} \mathrm{T}$ cell infiltration into tumors. These results demonstrate a critical role for 
BLT1 and CXCR3 in CTL migration to tumors and thus can be targeted to enhance effective anti-tumor responses. 


\section{TABLE OF CONTENTS}

CONTENTS

PAGE

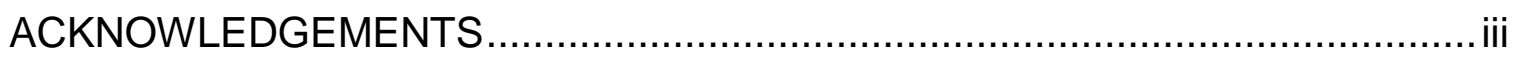

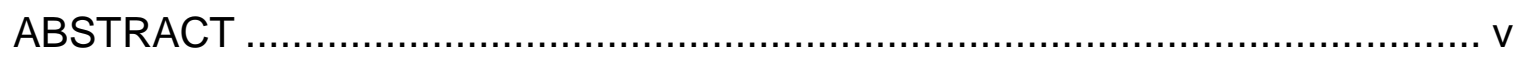

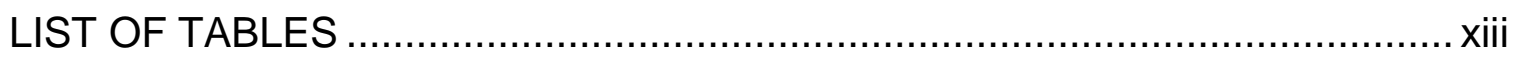

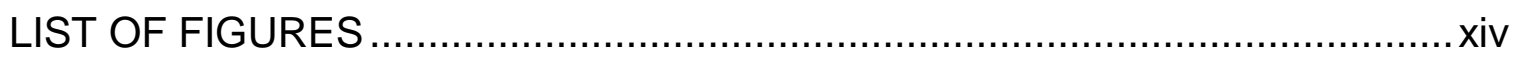

CHAPTER I

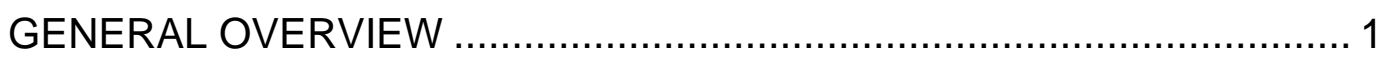

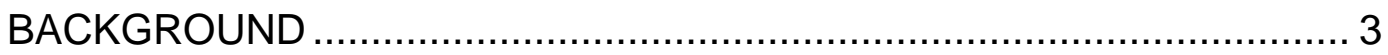

Dual role of immune system in tumor development .............................. 4

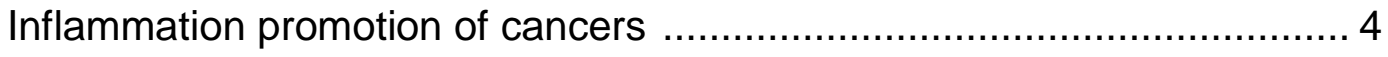

Immunesurveillance and Immunoediting of cancers .............................. 6

Cancer Treatment Approaches..................................................... 10

Immunotherapy as cancer treatment strategy .................................... 12

Reasons behind partial efficacy of immunotherapies............................ 20 
Chemoattractants and leukocyte migration

Chemokines regulate cancer development

Chemoattractants recruit activated $\mathrm{CD} 8^{+} \mathrm{T}$ cells to

target tissues

Chemokine receptors controlling activated CTL migration

to tumors

Chemoattractants and their receptors as targets for enhancing

CTL recruitment

Barriers to CTL infiltration to tumors 37

Leukotriene $\mathrm{B}_{4}$ and its high affinity receptor $\mathrm{BLT1}$ 40

\section{CHAPTER II}

BLT1 regulates anti-tumor immunity by mediating CTL infiltration to

tumors

CHAPTER III

MATERIALS AND METHODS

Mice and cell lines

Reagents 
Tumor model and vaccinations

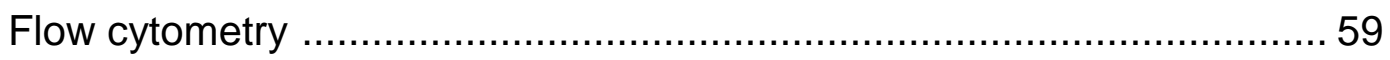

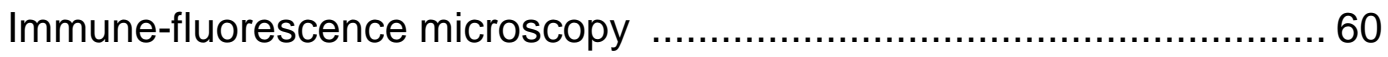

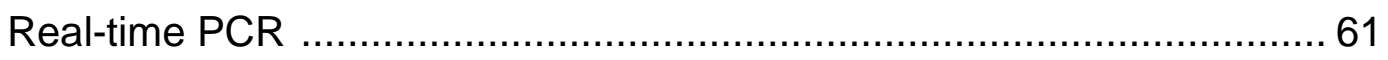

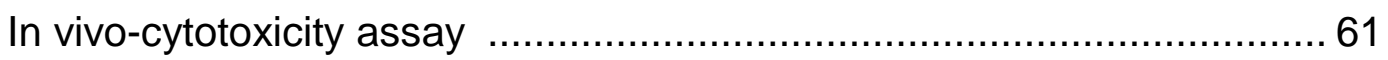

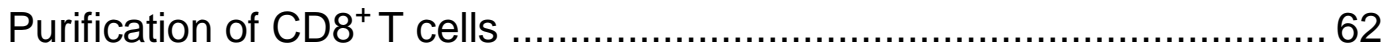

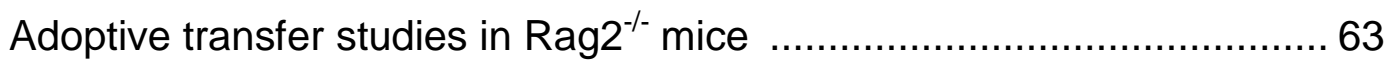

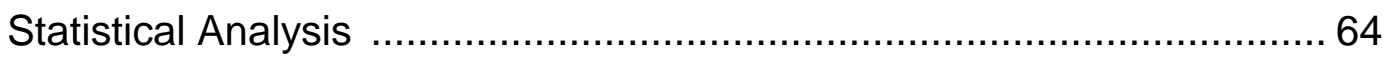

\section{CHAPTER IV}

BLT1 and CXCR3 regulate anti-tumor immunity by facilitating

$\mathrm{CD}^{+} \mathrm{T}$ cell migration to tumors

INTRODUCTION

65

RESULTS

Defective immune surveillance and anti-tumor immunity in BLT1 ${ }^{-/}$ and $\mathrm{CXCR}^{-/}$mice 67

Reduced homing of $\mathrm{CD}^{+} \mathrm{T}$ cells into tumors of $\mathrm{BLT1}^{-{ }^{-}}$and

$\mathrm{CXCR}^{-/-}$mice 68 
Effector responses in $\mathrm{WT}, \mathrm{BLT} 1^{-/-}$and $\mathrm{CXCR} 3^{-/-}$mice

Effector responses of WT, BLT1 $1^{-/}$and $\mathrm{CXCR}^{-/-} \mathrm{CD} 8^{+} \mathrm{T}$ cells

Adoptively transferred WT but not $\mathrm{BLT}^{-1-}$ or $\mathrm{CXCR}^{-1-} \mathrm{CD}^{+} \mathrm{T}$ cells

delayed tumor growth in $\mathrm{Rag}^{-{ }^{--}}$mice

\begin{abstract}
CHAPTER V
Lack of BLT1 and CXCR3 mediated synergism to facilitate CTL

migration to tumors and anti-tumor immunity
\end{abstract}

INTRODUCTION

RESULTS

Generation of $\mathrm{BLT}^{-1-} \mathrm{CXCR}^{-/-}$mice

Accelerated tumor growth and reduced survival in

$\mathrm{BLT}^{-1-} \mathrm{CXCR}^{-/-}$mice 90

WT CTLs do not facilitate knockout CTL infiltration

to tumors 


\section{CHAPTER VI}

PD-1 blockade based vaccine fails in $\mathrm{BLT1}^{-{ }^{--}}, \mathrm{CXCR}^{-{ }^{--}}$and

$\mathrm{BLT}^{-1-} \mathrm{CXCR}^{-/-}$mice

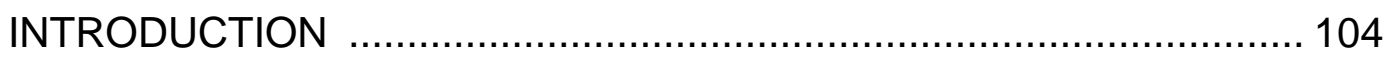

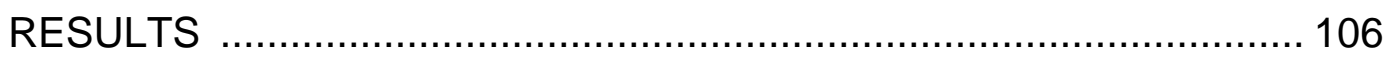

Obligate requirement for BLT1 and CXCR3 in anti-PD-1 Ab based

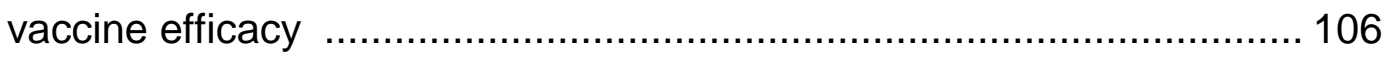

CHAPTER VII

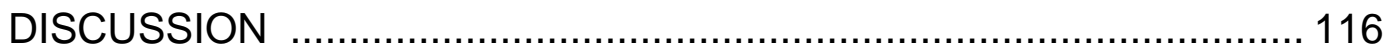

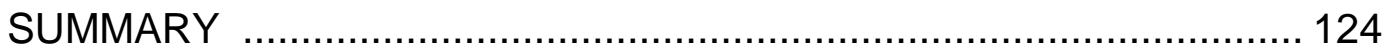

IMPLICATIONS AND FUTURE DIRECTIONS ................................. 128

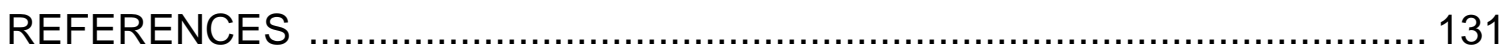

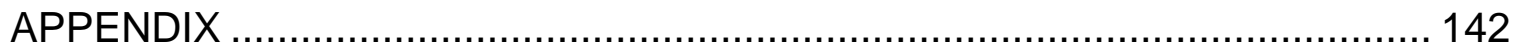

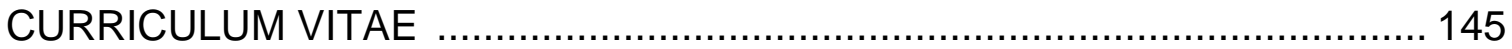




\section{LIST OF TABLES}

\section{TABLE}

PAGE

1. Breeding scheme for generation of $\mathrm{BLT}^{-1-} \mathrm{CXCR}^{-/}$mice 93 


\section{LIST OF FIGURES}

FIGURES

PAGE

1. The cancer Immunoediting concept

2. Chemokine network in the anti-tumor immune response

3. Decreased survival and increased tumor growth in $\mathrm{BLT}^{-/-}$mice

4. Reduced infiltration of effector anti-tumor immune cells in TC-1

tumors growing in $\mathrm{BLT} 1^{-/}$mice

5. Unaltered tumor growth and intratumoral NK cell numbers in Rag2 ${ }^{-1-}$

and $\mathrm{Rag} 2^{-/} \mathrm{BLT1} 1^{-/}$mice 51

6. Depletion of $\mathrm{CD}^{+} \mathrm{T}$ cells accelerates $\mathrm{TC}-1$ tumor growth in $\mathrm{BLT} 1^{+/+}$

but not in $\mathrm{BLT} 1^{-/}$mice

7. Adoptive transfer of tumor primed $\mathrm{CD} 8^{+} \mathrm{T}$ cells from $\mathrm{BLT} 1^{+/+}$but

not from $\mathrm{BLT1}^{-/-}$mice retards tumor growth in $\mathrm{Rag}^{-{ }^{--}}$mice 53

8. Chemokine receptor expression on the adoptively transferred $C D 8^{+}$

T cells from TDLNs and tumors 54

9. Enhanced tumor growth and reduced survival in $\mathrm{BLT}^{-1-}$ and $\mathrm{CXCR}^{-1-}$ 
Mice

10. Reduced infiltration of $\mathrm{CD} 8^{+} \mathrm{T}$ cells in $\mathrm{BLT} 1^{-/-}$and $\mathrm{CXCR} 3^{-/}$tumors 75

11. Similar numbers of $\mathrm{CD} 8^{+} \mathrm{T}$ cells in spleens of $\mathrm{WT}, \mathrm{BLT}^{-1-}$ and $\mathrm{CXCR}^{-/-}$tumor bearing mice 77

12. Similar numbers of $\mathrm{CD}^{+} \mathrm{T}$ cells in TdLN of WT, $\mathrm{BLT} 1^{-1-}$ and $\mathrm{CXCR}^{-/-}$tumor bearing mice 78

13. Similar numbers of myeloid cell subsets in tumors of WT, BLT1 ${ }^{-1-}$ and $\mathrm{CXCR}^{-/-}$mice 79

14. Effector responses in tumors of WT, $\mathrm{BLT} 1^{-1-}$ and $\mathrm{CXCR} 3^{-/}$mice 80

15. $\mathrm{BLT}^{-1-}$ and $\mathrm{CXCR} 3^{-/-} \mathrm{CD} 8+\mathrm{T}$ cells display normal cytotoxic function in the spleen. 81

16. Effector functions of WT, BLT1 ${ }^{-1-}$ and $\mathrm{CXCR} 3^{-/-} \mathrm{CD} 8^{+} \mathrm{T}$ cells in periphery versus tumor. 82

17. Adoptive transfer of $\mathrm{WT}$ but not $\mathrm{BLT1} 1^{--}$tumor experienced $\mathrm{CD} 8^{+} \mathrm{T}$ cells reduced tumor growth in $\mathrm{Rag}^{-/-}$mice 84

18. Adoptive transfer of WT but not $\mathrm{CXCR} 3^{-/-}$tumor experienced $\mathrm{CD}^{+} \mathrm{T}$ cells Reduced tumor growth in Rag2 ${ }^{-/-}$mice 86 
19. Defective immune surveillance and anti-tumor immunity in

$\mathrm{BLT}^{-/-} \mathrm{CXCR}^{-/-}$double knockout mice (DKO)

20. Lack of synergism in BLT1 and CXCR3 mediated regulation in anti-tumor immunity 96

21. Equal proportions of WT (GFP) and knockout $C D 8^{+} T$ cells adoptively transferred in tumor bearing Rag2 $2^{-/-}$mice. 97

22. Equal proportions of WT and knockout $C D 8^{+} \mathrm{T}$ cells in the TdLN of Rag2 $^{-/}$mice. 99

23. WT CD8 ${ }^{+} T$ cells do not facilitate knockout $C D 8^{+} T$ cell infiltration to tumors 101

24. $\mathrm{CXCR}^{-1}$ and DKO CD8 ${ }^{+}$T cells are significantly more as compared to WT cells in blood and spleen of tumor bearing Rag2 $2^{--}$mice 103

25. Obligate requirement of BLT1 and CXCR3 for optimum efficacy of anti-PD-1 antibody based immunotherapy 108

26. Vaccine enhances $C D 8^{+} \mathrm{T}$ cell infiltration in tumors of WT mice but not knockout mice 110

27. Vaccine enhances $\mathrm{CD} 8^{+} \mathrm{T}$ cell infiltration and effector molecules 
in tumors of WT mice but not knockout mice.

28. Enhanced expression of BLT1 and CXCR3 on $\mathrm{CD} 8^{+} \mathrm{T}$ cells upon

Vaccination. 114

29. A model for BLT1 and CXCR3 mediated regulation of anti-tumor

Immunity 


\section{CHAPTER I}

\section{GENERAL OVERVIEW}

Emerging data clearly demonstrates that the immune system can curtail cancer progression, a concept termed "Immune-surveillance against tumors". CD8 ${ }^{+} \mathrm{T}$ cells act as sentinels against tumors by directly eliminating the tumor cells. The prime focus of this research is to understand the mechanisms regulating $\mathrm{CD} 8^{+} \mathrm{T}$ cell migration to tumors with a particular emphasis on chemo-attractant receptors. The chapter begins with a brief description of the dual role of immune system in regulating as well as sculpting the tumor, a phenomenon known as immunoediting. The cellular and molecular mechanisms involved in killing tumor cells and how this knowledge is translated into various successful immunotherapies that have revolutionized the field of cancer therapies is described in greater detail with a particular focus on PD-1 blockade based therapies, Adoptive T cell therapies (ACT) and Chimeric Antigen Receptor (CAR) based therapies. Reasons behind failure of immunotherapies in cancer patients is discussed, defective $\mathrm{CD} 8^{+} \mathrm{T}$ cell infiltration to tumors being a major one as well as hurdles in achieving optimum $\mathrm{CD}^{+} \mathrm{T}$ cell infiltration to tumors.

The importance of chemokines and their cognate receptors in regulating cancer development and $\mathrm{CD}^{+} \mathrm{T}$ cell infiltration to tumors is discussed. Furthermore, we 
outlined the background on Leukotriene $\mathrm{B}_{4}$ receptor $\mathrm{BLT1}$; and $\mathrm{CXCR} 3$, receptor for CXCL9 and CXCL10, that are studied in detail in this thesis. 


\section{BACKGROUND}

Transformed cells arise due to genetic mutations resulting in uncontrolled cellular divisions. Tumor growth is a cumulative effect of three steps viz. initiation of a cancerous event in a cell, promotion of the cancerous event by proliferation and finally progression involving tumor growth and metastases [1]. Cancer development requires attainment of six hallmark capabilities: self-sufficiency in proliferation, insensitivity to anti-proliferative signals, evasion of apoptosis, unlimited replicative potential, the maintenance of vascularization and tissue invasion and metastasis [2].

Multiple intrinsic and extrinsic mechanisms exist to prevent such aberrant cell divisions. These "intrinsic" cellular mechanisms protect the cells against various perturbed mutagenic insults or cell stresses including hypoxia, nutrient deprivation, DNA damage, tissue injury etc. Should the cell proliferation go beyond control, the cell activates apoptosis or cell death pathways through activation of pro-apoptotic machinery, engagement of death receptors, activation of tumor suppressor genes like p53, etc. If the transformed cells escape the intrinsic control, they are then subjected to the "extrinsic" mechanisms wherein neighboring cells sense the presence of a transformed cell and try to eliminate it. Dependency of cells on trophic environmental cell-cell contact signals or extracellular matrix (ECM) dependence; genes controlling cellular junctions and polarity and finally detection and elimination of transformed cells by the immune system; constitute the three extrinsic mechanisms to prevent the maintenance of an oncogenic event [3]. 


\section{Dual role of immune system in tumor development}

The role of immune system in controlling cancer has long been a subject of debate. The initial clues for the involvement of immune system in cancer growth stemmed through the observation made by Virchow in 1863 that tumor biopsy samples contain leukocytes termed as "lymphoreticular infiltrate". On the other hand, as far back as 1700s the observations that feverish infections in cancer patients occasionally led to cancer remissions suggested that immune activation and cancer remission might be associated. The very first attempt at using immunotherapies as cancer therapies was made by W. Coley in 1893 wherein he injected killed bacterial mixture of Streptococcus pyogenes and Serratia marcescens to cancer patients and observed tumor regression in some cases [4]. Since then several studies have later proven that immune system can curtail tumor growth as well as promote it.

\section{Inflammation promotion of cancers}

Recent studies have expanded the concept that inflammation plays a crucial role in tumor promotion. Only $10-15 \%$ of the cancers are hereditary and the rest are caused by somatic mutations at sites of infection, exposure to chemical irritant, cigarette smoke, environmental and dietary factors. The fact that patients suffering from inflammatory bowel disease or Crohn's disease often develop colon cancers strongly correlates chronic inflammation with cancer development [5]. Patients positive for Hepatitis C infection and Helicobacter pylori infections

are predisposed to liver cancer and stomach cancer development respectively, 
associating infections to cancers [6, 7]. The use of Non-steroidal antiinflammatory drugs (NSAIDs) in the prevention of spontaneous tumors in Familial Adenomatous Polyposis (FAP) patients also establishes a strong connection between inflammation and cancer [8].

Oncogenic event also occurs due to mutations caused by reactive oxygen species and nitrogen intermediates (ROS and RNI) as well as superoxide and hydroxyl radicals produced as a result of inflammation [9]. Studies on the role of NF-kB have provided further insights in the participation of inflammation in tumor growth. Murine model of colitis-associated cancer involves azoxymethane (AOMpre-carcinogen) mediated induction of an oncogenic event that by itself gives rise to fewer numbers of adenomas along the intestines. The adenomas can be augmented by simultaneous induction of colonic inflammation through repeated exposure to intestinal irritant dextran sulfate sodium (DSS). Inactivation of NF-kB pathway in colonic epithelial cells in an AOM-DSS model of intestinal tumorigenesis resulted in a significant reduction in the tumor incidence [10]. NFkB transcription factor was not only shown to be a key component of inflammation but also known to facilitate the survival of the initiated colonic epithelial cells. It was also involved in activating the myeloid immune cells to produce inflammatory cytokines like IL-1 $\beta$, IL-6, TNF $\alpha$, IL-12/IL-23; chemokines like KC, MIP-2, and inflammatory mediators like COX-2, STAT-3, MMP-9, etc. that promote the growth of cancerous cells. Ablation of NF-kB activity in protumorigenic tumor associated macrophages (TAMs) re-educated them to gain cytotoxic, anti-tumorigenic potential [11]. Tumor cells also feed into the 
inflammation by secreting factors that allow the migration of various other inflammatory myeloid cells that promote the tumor growth. For example, COX-2 is frequently expressed in tumor cells and is involved in the synthesis of prostaglandins and chemokines like IL-8, CCL2, CCL20, up-regulating the chemokine receptor CXCR4 that binds to the chemokine CXCL12 or SDF-1 (stromal cell derived factor-1), activating matrix degrading enzymes, etc. [9]. Tumor suppressor genes are also involved in activating inflammatory pathways in tumor cells. For example, von Hippel-Lindau tumor suppressor protein (VHL) targets the transcription factor Hif-1a or hypoxia-inducible factor $1 \alpha$ for degradation. The role of HIF-1a in cellular response to hypoxia is very well established. Some of the functions of Hif-1a include initiating angiogenesis, interaction with NF-kB to promote TNFa production and is also known to help express CXCR4 chemokine receptor on tumor cells involved in metastasis [12].

\section{Immunesurveillance and Immunoediting of cancer}

Despite the semantics of correlation between inflammation and cancer, the immune system is still the third extrinsic mechanism to prevent tumorigenesis. In fact the pro-tumorigenic effects of immune system emerge if the cytotoxic immune cells do not eliminate the cancer cells. The notion that cancerous cells would emerge at an "incredible frequency" if the host defenses would not prevent the growth of continuously arising cancer cells was conceived in 1909 by Paul Erlich. Fifty years later Burnett and Thomas predicted that lymphocytes were responsible for eliminating the nascent tumor cells and hence introduced the concept of "immune-surveillance" against tumors [3]. Since then there has been 
unequivocal evidence reinforcing the beneficial role of the immune system in eliminating the tumor cells. By 1990s, the availability of better immunodeficient murine models on pure genetic background led to the importance of interferon gamma (IFNY) and STAT1 (transcription factor required for IFNy signaling) in rejection of transplanted tumors. This rekindled the role of lymphocytes in antitumor immunity as demonstrated in spontaneously arising tumors as well as chemically induced tumors $[13,14]$. The results obtained in immunecompromised mice led to the paradox of tumor formation in immune-competent mice/individuals which led to the concept of "Cancer immunoediting" pioneered by Schreiber and colleagues that describes the dual roles of the immune system in three sequential steps of tumor progression namely Elimination, Equilibrium and Escape phases [13, 15].

In the Elimination phase the innate (dendritic cells, macrophages, NK cells) and the adaptive immune cells $\left(\mathrm{CD} 8^{+}\right.$and $\mathrm{CD} 4^{+} \mathrm{T}$ cells, $\mathrm{y} \delta \mathrm{T}$ cells) work in concert towards eliminating any nascent tumor cells. Release of effector cytokines like Type I (IFN $\alpha$ and IFN $\beta$ ) and II (IFNY) interferons, TNF $\alpha$, IL-12 by the innate cells upon recognition of tumor antigens recruit the effector cells including effector $\mathrm{CD}^{+}$and $\mathrm{CD}^{+} \mathrm{T}$ cells as well as NK cells to the tumors. The CTLs themselves further express granzyme-B, perforin, TRAIL, Fas/FasL, IL-2, IFNy, TNFa, NKG2D, IL-17 etc. that directly kill the tumor cells and enhance the antigenicity of tumor cells by enhancing the MHC-I expression on tumor cells $[3,16]$.

In the Equilibrium phase, the tumor cells and immune cells exist in a state of equilibrium. At this stage, tumor growth can proceed in either direction - 
elimination or escape. Effector cells (T cells) and cytokines (IFNy, IL-12) are indispensable in this phase and there is a balance established between antitumor and pro-tumor immune mechanisms $[16,17]$. The immune cells can keep the tumors in a functionally dormant state, wherein tumor cells acquire genetic mutation because of the immune selective pressure leading to the generation of "tumor variants". The tumor cell variants that emerge in the previous phase grow to become resistant to immune detection or elimination and become tolerized by establishing various immune-suppression mechanisms that allow the tumors to grow and become clinically detectable.

The tumor now progresses to the next phase, Escape. The tumor-induced mechanisms include tumor cells acquiring additional somatic mutation, loss of response to IFNY, loss of antigen presentation due to downregulation of MHC-I molecule on tumor cells, enabling infiltration of immune-suppressive cells as well as expression of immune-suppressive markers like PD-L1 on tumor cells. The immune induced mechanisms include subversion of $T$ cell responses, $T$ cell anergy, immune suppression via myeloid cells like TAMs, MDSCs (myeloid derived suppressor cells) as well as regulatory T cells (Treg) that express various immune suppressive factors like TGF $\beta$, IL-10, IDO (indoleamine 2,3dioxygenase), FasL, galectin, VEGF, PD-1/PDL1, CTLA4 etc. [3, 16]. The amount of literature in understanding these three phases of immunoediting has been burgeoning in the past decade. The final outcome of tumor growth depends on whether tumor promoting or tumor suppressing inflammation dominates. 


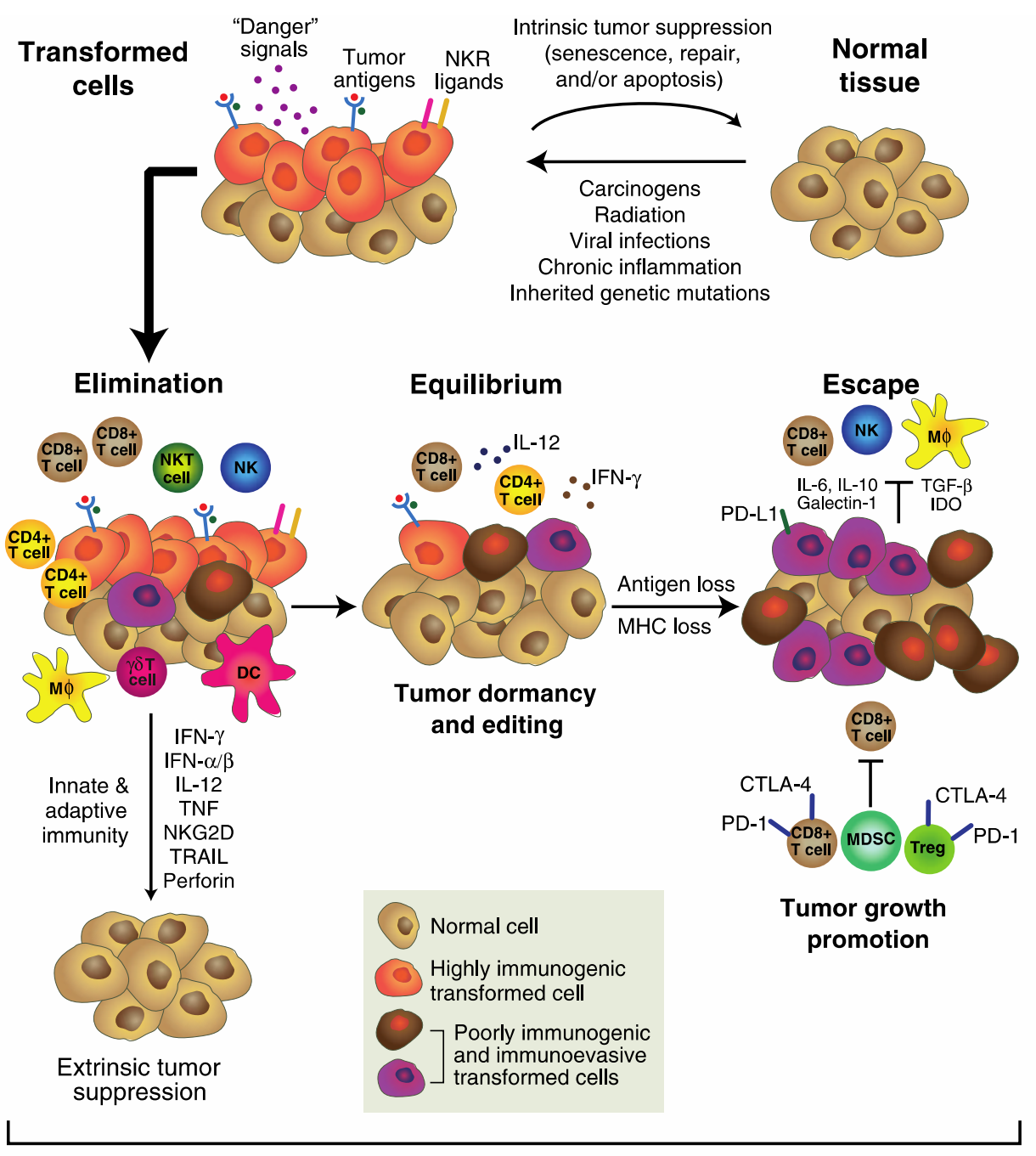

Cancer Immunoediting

Figure 1: The cancer immunoediting concept. Cancer immunoediting is an extrinsic tumor suppressor mechanism that engages only after cellular transformation has occurred and intrinsic tumor suppressor mechanisms have failed. In its most complex form, cancer immunoediting consists of three sequential phases: elimination, equilibrium, and escape.

Figure adapted from [18]. 
Patients with established cancers indicate the failure of immune surveillance mechanisms. The immune cells that are present in the tumors of these patients now actually promote the tumor growth by releasing pro-tumorigenic cytokines and chemokines. The conventional tumor therapies like radiotherapy and chemotherapies were originally aimed at killing the cancer cells directly; however, recent studies have demonstrated that these therapies are also immunomodulatory in nature. Given the beneficial role of immune surveillance in cancer,

attempts are being made to jumpstart the anti-tumor responses. Therefore a major focus of the field of tumor immunology is to modulate the immune system such that the anti-tumor responses get activated and pro-tumorigenic responses get subverted.

\section{Cancer Treatment Approaches}

Surgery, radiotherapy and chemotherapy are the three most common standard of care treatment approached to most of the cancers. Most of the chemotherapy drugs as well as radiotherapy induce cancer killing by inducing apoptosis in cancer cells. While offering short term benefit, the standard of care treatments can lead to more aggressive cancer cells that become resistant to both chemotherapy and radiotherapy possibly due to disruption of intrinsic apoptosis machinery. One of the reasons behind the aggressiveness of cancer cells post therapy can be due to the negative effect of the radiation and chemotherapy on the immune system. It is now known that certain chemotherapy drugs that targeted cancer cells based on the rapid cell division criterion, also targeted the rapidly dividing hematopoietic cells including immune cells, thus leading to 
immunosuppression. Total body irradiation leads to ablation of patient's immune system. Radiation was also shown to promote immunosuppressive mechanisms like activation of transforming growth factor- $\beta$ and enhancing pro-tumorigenic function of macrophages. Also Treg cells are more resistant to radiation induced death compared to other $\mathrm{T}$ cells which leads to presence of more Treg compared to CTLs in the tumor.

Recent studies have demonstrated that the standard of care therapies can have positive impact on immune system. Certain chemotherapy drugs and localized radiotherapy can induce immunogenic cell death, thus enhancing anti-tumor immunity $[19,20]$. Local tumor irradiation instead of total body irradiation has recently shown to benefit the anti-tumor effect of the immune system. Even chemotherapies like cyclophosphamide and 5-fluorouracil are considered to be immune-modulatory in nature by eliminating suppressive Treg and MDSC immune cells thereby enhancing anti-tumor immunity. Hence radiotherapy and certain chemotherapy drugs can induce immunogenic cell death mechanisms that involve the activation of type-I IFNs that lead to activation of innate and adaptive system against tumors. Also, immunogenic cell death induces release of various tumor antigens/neo-antigens that act as endogenous vaccines [21-26]. This provides mechanistic rationale behind combining specific chemotherapies and/or radiation therapy with existing immunotherapies, thus opening up new horizons for the treatment of cancers. 


\section{Immunotherapy as cancer treatment strategy}

Immunotherapies have only recently emerged as a successful treatment modality for cancer [27-29]. Various studies indicated that adjuvants, lectins, IL-2 and interferons could target the tumor cells by activating the lymphocytes [30-32]. A series of landmark approvals by the FDA including a) IFN 2 2 for the adjuvant treatment of stage IIB/III melanoma in 1995 , b) IL-2 for the treatment of metastatic melanoma and renal cell carcinoma in 1998, c) first ever cancer vaccine - "Oncophage/vitespen" for treatment of renal cell carcinoma in Russia in 2008, d) approval of autologous Dendritic cell cancer vaccine (Provenge) for Stage IV hormone refractory prostate cancer and e) immune checkpoint blockade strategies such as anti-CTLA4 (Ipilimumab) in 2011 for melanoma, and anti-PD-1 (Pembrolizumab) in 2014 for advanced metastatic melanoma and non small cell lung carcinoma; have all been significant in reinforcing that immunotherapies hold tremendous potential to combat tumors thus starting a new wave of revolution in cancer therapeutics [33-39]. Three major lines of immunotherapy approaches emerged in recent years against tumors viz. a) employing Adoptive cell transfer therapy including chimeric antigen receptor therapy, b) blocking immune checkpoint inhibitory pathways like PD-1, CTLA, TIM3 and LAG3, and c) activating T cell co-stimulatory pathways by using agonistic antibodies to OX-40, 4-1BB, ICOS. These immune-therapies have higher success rates due to increased specificity to cancer antigens as well as inducing long lasting $T$ cell responses and immunological memory. $T$ cell based therapies including anti-PD-L1 and anti-PD-L2 antagonistic antibodies, adoptive 
cell therapies including chimeric antigen receptor therapy, vaccine candidates like Prostvac targeting the prostate specific antigen (PSA) and various agonistic antibodies for co-stimulatory receptor candidates are currently in advanced clinical trials.

An effective $T$ cell dependent anti-tumor response involves five major steps: a) efficient antigen sampling by dendritic cells (DCs) and their maturation; b) migration of these DCs to tumor draining lymph nodes (TdLNs); c) recirculation/migration of naïve T cells to TdLNs; d) antigen presentation by the DCs to the $T$ cells, clonal expansion and acquisition of effector functions in $T$ cells and finally e) migration of these activated CTLs to tumors to execute their anti-tumor functions [40, 41]. Although all these steps are crucial and interconnected, the final outcome must be the presence of $\mathrm{CD} 8^{+} \mathrm{T}$ cells in tumors and with intact effector functions. Manipulating these steps of anti-tumor immunity has led to various successful immune-therapies against cancer. DC based vaccines, for example, accomplishes step 1 by enhancing the antigen loading and maturation ability of the DCs [34]. Immune checkpoint inhibitors i.e. anti-PD-1 and anti-CTLA4 antagonistic antibodies [39] and co-stimulatory agents (4-1BB, ICOS, GITR agonists, etc. [42]) as well as adjuvants (lectins, IL-2) [43] when used accomplishes step 4 by enhancing the effector functions of the CD8 ${ }^{+}$ T cells against tumor cells. Alternatively, immunotherapies like Adoptive $T$ cell therapies (ACT) including Chimeric Antigen Receptor (CAR) therapy bypass all the steps except the last step of infiltration of CTLs to tumors and anti-tumor 
function [44]. The most successful immunotherapies relevant to the current project specifically in melanoma are detailed below.

\section{Immune checkpoint blockade: anti-PD-1 and anti-CTLA4 antibodies}

Inhibitory receptors present on the immune cells attenuate the immune responses to prevent excessive inflammation that can be detrimental to the tissues. $T$ cell activation is a complex process and involves antigen specific stimulation via the TCR and a co-stimulatory signal. It is now increasingly clear that both co-stimulatory and co-inhibitory signals are required to maintain an effective $T$ cell response. Enhancing signaling through co-stimulatory molecules (4-1BB, GITR, OX-40) and blocking the co-inhibitory molecules (CTLA4, PD-1, LAG3) can amplify T cell responses to tumors [45-47].

CTLA4: CTLA4 is a type 1 transmembrane glycoprotein inhibitory receptor that is present on $T$ cells and belongs to of the CD28/Immunoglobulin superfamily. By binding to B7-1 (CD80) and B7-2 (CD86) on APCs, CTLA4 competes with CD28 costimulatory molecule to dampen $\mathrm{T}$ cell responses. Optimal crosslinking of CTLA4 with TCR-CD28 stimulation led to IL-2 suppression, proliferative arrest without induction of apoptosis. A monoclonal Ab was made by Allison and colleagues to specifically block CTLA4 in a preclinical model wherein significant anti-tumor immunity without any overt immune toxicity was achieved upon CTLA4 blockade [46]. Subsequently two fully humanized CTLA4 antagonistic antibody Ipilimumab and Tremilimumab were introduced in clinical trials in 2000 for metastatic melanoma. Ipilimumab was a better antibody and gave significant 
overall response rates of $17 \%$ in patients with advanced melanoma with three year survival rates being 20\% [48]. Subsequently, in 2010 Ipilimumab was the first immune-checkpoint blockade therapy that was FDA approved for metastatic melanoma [49].

PD-1: PD-1 was originally identified in 1992 as a gene induced upon apoptotic cell death in a T cell hybridoma [50]. Later studies demonstrated that PD-1 deficient mice showed signs of autoimmunity that suggested a role of PD-1 as an inhibitor of lymphocyte responses at peripheral tissues. Further insight on the role of PD-1 as an inhibitory receptor was gained from a chronic viral infection model $[51,52]$. While expressed minimally on resting immune cells, PD-1 is broadly expressed on T cells, B cells, NK cells, DCs and macrophages upon activation. PD-1 binds to its partners PD-L1 (B7-H1) and PD-L2 (B7-DC). PD-L1 is broadly expressed on cells of both hematopoietic and non-hematopoietic lineage including tumor cells. PD-L1 expression is induced upon inflammatory cytokines like TNFa and interferons. PD-L2 expression is restricted to immune cells like DCs, macrophages and mast cells. CTLA4 and PD-1 control T cell responses by different mechanisms. They both block activation of Akt thereby blocking glucose uptake by CD28. However, PD-1 blocks PI-3K activation while CTLA4 blocks Akt downstream of PI3K. Adoptively transferred T cells express PD-1 and numerous tumor cells express PD-L1 suggesting the role of this signaling pathway in tumor immune evasion [53]. In 2014, Pembrolizumab was the first anti-PD-1 drug approved by the FDA against relapsed or refractory melanoma. At six months, it was shown to enhance progression free survival by 
$30 \%$ as compared to $16 \%$ by chemotherapy. Nivolumab was the second anti-PD1 drug to be FDA approved for metastatic melanoma and metastatic squamous non-small cell lung carcinoma (NSCLC). It showed $72 \%$ overall survival rate at one year for metastatic melanoma patients without BRAF mutation [48]. PD-1 and CTLA4 combination blockade was shown to be more effective compared to either treatments alone [54]. Whole exome sequencing of non-small cell lung cancers treated with Pembrolizumab suggested that higher non-synonymous mutational landscape in lung tumors was associated with better objective response and clinical benefit [55]. The higher mutational burden also correlated with molecular smoking signature and increased neo-antigen burden [55]. This also explained why some patients were unresponsive to PD-1 blockade.

Similarly, studies suggest that only a subset of CRC, may be a good candidate to PD-1 blockade therapy [56]. Microsatellite instable (MSI) subset of colorectal cancer comprises $15 \%$ of sporadic CRC and most familial CRC [57]. MSI, typically diagnosed by variable length DNA microsatellites, are mutations arising due to epigenetic silencing of DNA mismatch repair genes [58]. The high mutational burden in MSI tumors creates many tumor-specific neo-antigens compared to microsatellite stable tumors [59]. MSI positive tumors correspond to higher level of tumor-infiltrating leukocytes, T cell responses, PD-1 and PD-L1 expression and hence better response to PD-1 blockade therapies [56, 57, 59].

Adoptive T cell therapy (ACT): This technology was pioneered by Dr. Steven Rosenberg and is now widely used in patients. ACT involves the transfer of ex- 
vivo activated, stimulated and expanded autologous $T$ cells with high affinity to tumor antigens back into the patients. Ex-vivo activation and culture of tumor specific $T$ cells is possible outside of the endogenous host that has immunesuppressive factors. Anti-tumor $\mathrm{T}$ cells with a $\mathrm{CCR} 7^{+} \mathrm{CD} 27^{+} \mathrm{CD}^{2} 8^{+} \mathrm{CD} 62 \mathrm{~L}^{+}$ phenotype characteristic of central memory cells were more effective in ACT protocol than more differentiated effector cells [60]. Antigen specific cells are isolated from the freshly excised tumors and as soon as the anti-tumor activity is detected against specific antigens, cells were expanded in the presence of $T$ cell stimulating antibody OKT3 and IL-2 $(6000 \mathrm{IU} / \mathrm{ml})$. Approximately $5 \times 10^{10}$ cells were infused systemically following a non-myeloablative preparative regimen consisting of $60 \mathrm{mg} / \mathrm{kg}$ cyclophosphamide for 2 days followed by 5 days of fludarabine at $25 \mathrm{mg} / \mathrm{m}^{2}$. IL2 was administered for $2-3$ days at $7.2 \times 10^{5} \mathrm{IU} / \mathrm{kg}$ every $8 \mathrm{hr}$ [61]. ACT today represents one of the most promising T cell based immunotherapy for melanoma, lymphoma and childhood leukemias. The source of the T cells could be PBMCs or tumor itself. The ACT procedure involves preconditioning the patient by temporary ablation of immune system or lymphodepletion through total body irradiation or chemotherapy for removal of regulatory $\mathrm{T}$ cells, competition for homeostatic cytokines like IL-7 and IL-15 involved in T cell survival and proliferation thereby enhancing the persistence of the transferred $T$ cells [62]. The lymphodepletion regimen before ACT when combined with administering T cell growth factor IL-2 led to tumor eradication for prolonged durations [62-64]. Objective response rates for this therapy against Stage IV melanoma were between $49-72 \%$. Importantly, 22\% of the patients had 
complete tumor regression and disease free survival for more than 8 yrs [62]. Data from tumor exomic sequencing have enabled the identification and targeting of numerous non-synonymous mutations that result in new epitope generation [65]. ACT also includes TCR gene modified T cells against shared tumor differentiation antigens like MART-1, Carcinoembryonic antigen (CEA), cancer germ line antigens like NY-ESO-1, MAGE-A3 that have shown considerable success in various clinical trials [44]. Despite the success of ACT, a multitude of patients remain unresponsive. Upregulation of immune-suppressive molecules on the transferred CTLs (CTLA4 and PD-1), immune-suppressive tumor microenvironment (IDO, NOS-1), and the nature of tumor vasculature are some of the reasons behind differential response rates of ACT. Attempts are being made to utilize T cells with TCR against a specific antigen or patient specific tumor antigen called neo-antigens based on mutations in tumor DNA, identify $T$ cell subsets to be used for ACT (Naïve, Tcm, Tscm, Tem), identify T cell growth factors used (IL-2, IL-7, IL-15) to enhance persistence, proliferation and survival of $\mathrm{T}$ cells, and understand better host preconditioning protocols for lymphodepletion and T cell engraftment [66-69]. Studies are now undertaken to understand specific markers to identify and sort out from the tumor immune infiltrate that would give better anti-tumor responses in an adoptive cell transfer setting. Recent studies showed that $\mathrm{PD} 1^{+} \mathrm{CD} 8^{+} \mathrm{T}$ cells and not $\mathrm{PD} 1^{-} \mathrm{CD} 8^{+} \mathrm{T}$ cells represent the cohort of clonally expanded tumor reactive TILs and included T cells that target mutated tumor antigens [70]. 
Chimeric Antigen Receptor therapy: In line with ACT, Chimeric Antigen Receptor therapy (CAR) is another immunotherapy that has shown considerable success with hematological cancers. It involves genetic modification of $T$ cells such that they stably express chimeric antibodies conferred with antigen specificity. Briefly, chimeric antigen receptor is a fusion of antigen recognition domain of an antibody with the intracellular domain of CD3zeta chain or FcyRI. These first generation CARs effectively demonstrated cytotoxicity and $\mathrm{T}$ cell activation but failed to show proliferation and survival of $\mathrm{T}$ cells upon continuous antigen exposure. Studies suggested that the first generation CARs become anergic in the absence of co-stimulation in the tumor mileu. Second and third generation CARs encompassed the combination of CD3zeta chains with costimulatory molecules like CD28, CD27, 4-1BB and OX-40 [71, 72]. The antigen specificity and HLA independent recognition are some of the benefits of using CARs. Autologous T cells transduced to express CD19 CARs alongwith 4-1BB co-stimulatory molecule have shown considerable success with $50 \%$ of them achieving partial or complete remissions and T cells persisting beyond two years in patients with refractory and relapsed B cell chronic lymphocytic leukemia [73]. However, there can be various side effects like cytokine release syndrome and tumor lysis syndrome and B cell aplasia. Nonetheless, there is an increasing excitement with gene modified T cells entering cancer therapeutics. 


\section{Reasons behind partial efficacy of immunotherapies}

The past decade has witnessed a major revolution in immunotherapy as a promising treatment modality for cancer. Despite the tremendous success of immunotherapies, large numbers of cancer patients still remain unresponsive. For example, PD-1 blockade therapy leads to tumor progression free survival in only $30 \%$ of the patients while ACT treatments lead to complete tumor regression in only $22 \%$ of the patients $[48,62]$. The reason behind this is probably multifold. As indicated before the process of anti-tumor immunity requires proper execution of various steps and involvement of various cells. Any immunotherapy approach would have to overcome various hurdles. For example, "antigenicity" of the tumor is an important measure for potential susceptibility to immune therapies. Tumor can express variety of non-mutated or mutated antigens or even lose the antigenicity by reducing the antigen presentation via MHC-I downregulation or dysregulation in antigen presentation machinery. Although recent studies have demonstrated that immune response can be mounted against the neo-antigens or the antigens derived from somatic mutations that are specific to cancer cells (and not normal cells); the mutational landscape could be quite different across every individual that complicates the use of immunotherapies to the generalized population. Also the efficacy of immunotherapies to neo-antigens suggest the possibility that higher number of mutations correlate with enhanced response to immunotherapy [74]. Importantly, the failure of immunotherapies in some patients stems through the off-target toxic side-effects from the use of immunotherapies like CTLA4 blockade, CAR therapies etc. Liver toxicities, respiratory distress 
syndrome, cytokine storm syndrome are the various side-effects that limit the efficacy of the T cell based immunotherapies.

Studies have revealed that some immunotherapies including cancer vaccines are able to mount anti-tumor responses in the periphery but not in the tumor. Tumor mileu being inherently tumor suppressive is a major obstacle in success of immunotherapies. Immunotherapies that boost $\mathrm{T}$ cell responses fail to maintain the anti-tumor responses in the tumor due to immune-suppressive cells, cytokines, growth factors, hypoxic environment, etc. that inactivate or subvert the T cell based responses [41].

Another major impediment acknowledged only recently is defective $\mathrm{CD} 8^{+} \mathrm{T}$ cell infiltration to tumors despite effective responses in the periphery $[40,75]$. Studies have demonstrated that less than $2 \%$ of adoptively transferred $\mathrm{T}$ cells actually reach the tumor $[76,77]$. Majority of the transferred cells remain in the periphery or are found in lung and liver, which may also be a reason behind off-target responses and toxicities seen in $\mathrm{T}$ cell based therapies. Enhancing effector $\mathrm{T}$ cell recruitment to tumors by employing chemokine/chemokine receptor pathways is a crucial strategy to circumvent these issues. For an effective immune response to occur it is of importance that specific immune cells (anti-tumorigenic and not pro-tumorigenic) be recruited at specific locations (tumors versus the periphery). A major focus of this thesis is to understand how chemoattractant receptors regulate the efficacy of immunotherapies. Indeed studies conducted herein suggest that absence of BLT1 and CXCR3 chemoattractant receptors completely abrogates anti-PD-1 based vaccine efficacy and therefore suggests an 
indispensable role for both BLT1 and CXCR3 in achieving optimum efficacy of PD-1 blockade based immunotherapy.

\section{Chemoattractants and leukocyte migration}

Leukocyte migration involving both homeostatic recirculation among lymphoid organs and migration to inflamed sites including tumors is orchestrated and tightly regulated by various chemo-attractants. Chemoattractants include lipids, peptides as well as proteins. These include classic neutrophil chemoattractant peptides such as fMLP that is a tripeptide of $\mathrm{N}$-formylmethionyl-leucylphenylalanine (fMet-Leu-Phe) [78]; complement cleavage products C5a and C3a that are involved in migration of various leukocytes [79] and lipid chemoattractants like leukotriene $\mathrm{B}_{4}\left(\mathrm{LTB}_{4}\right)$ and platelet activating factor (PAF) identified as strong chemoattractants for neutrophils. In addition a large family of protein chemoattractants or chemokines broadly divided into four groups i.e. CC, $\mathrm{CXC}, \mathrm{CX} 3 \mathrm{C}$ and the $\mathrm{XC}$ families based on the number and position of vicinal cysteine residues are potent chemoattractants for various leukocytes $[40,80]$. Chemokines bind to G-protein coupled receptors with seven transmembrane domains. Differential receptor expression on immune cells is a crucial factor in determining responsiveness to chemokines. Around 50 chemokines and 20 chemokine receptors have been identified to date [81]. This suggests that various chemokines and chemokine receptors bind to multiple counterparts suggesting functional redundancy and complex regulation of cell migration [82]. No chemokine is uniquely active on one particular leukocyte subset [83]. Chemokines can be produced by a variety of immune cells/endothelial 
cells/epithelial cells but specific cell types are involved in chemokine production under specific inflammatory conditions. Usually, multiple chemokines are produced concomitantly in a redundant manner in response to the same stimulus by a cell, this phenomenon referred to as "polyspeirism" [83]. Two general modes of chemokine production; constitutive production (eg. SDF-1, CCL19, CCL13, CCL17, CCL25, etc.) or inducible production upon activation (eg. CXCL10, CCL5, CCL3, CCL4, CCL2, IL-8, etc.) are defined [83]. Leukocyte migration is tightly regulated by spatial and temporal expression of chemokines [84].

\section{Chemokines regulate cancer development}

It is increasingly evident that a tumor is a complex microenvironment that constitutes various cell types including immune cells, stromal cells like fibroblasts, tumor cells and endothelial cells that communicate with each other. The composition of the tumor microenvironment in terms of the types of immune cells recruited decides whether anti-tumor or pro-tumor immune responses predominate and ultimately the fate of tumor development. Chemokine/cytokine mediated inflammation plays a crucial role in initiation and progression of various cancers [85]. Tumor cells also produce inflammatory chemokines and express chemokine receptors. Melanoma cells have shown to express various chemokines like CCL2, CCL5, CXCL1, CXCL2, CXCL3 and CXCL8 that are protumorigenic $[81,86]$. It is well known that various cancer cells express CXCR4, chemokine receptor to SDF-1 and regulates the growth, migration and invasion of tumor cells by activating AKT, MAPK, and JAK-STAT pathways [87, 88]. Apart from CXCR4-SDF-1 axis, various other chemokines and their receptors have 
been involved in angiogenesis and metastasis of cancer as well. Chemokines like CXCL1, CXCL2, CXCL3, CXCL5, CXCL6, CXCL7, and CXCL8 activating CXCR1 and CXCR2 receptors have been shown to be promoters of angiogenesis [81].

With respect to immune cells in the tumor mileu, CXCR3 receptor and its ligands CXCL9, and CXCL10 are strongly related to Th1 biased responses that is a crucial part of effector anti-tumor responses [89-91]. CXCR3-CXCL9/10 pathway is an important axis involved in $\mathrm{CD}^{+}, \mathrm{CD}^{+} \mathrm{T}$ cell and $\mathrm{NK}$ cell infiltration to tumors for anti-tumor immunity. CXCR3 expression was also shown to be crucial to generate cytotoxic anti-tumorigenic M1 polarized macrophages [92]. Another chemokine receptor involved in $\mathrm{CD}^{+} \mathrm{T}$ cell mediated antitumor immunity is CCR5. CCR5 on CD4 and CD8 ${ }^{+} \mathrm{T}$ cells was shown to be crucial in anti-tumor responses as deficiency in CCR5 led to enhanced lung adenocarcinoma progression [81, 93].

Tumor promoting cells are also governed through chemoattractant pathways. It is known that tumors shape the microenvironment to its advantage by recruiting immune suppressive cells like Treg cells, TAMs, MDSCs etc. Treg cells constitute a considerable portion of the tumor mileu and through the production of immune suppressive molecules like IL-10 and TGF- $\beta$ are extremely potent in dampening the anti-tumor response [94]. CCR4-CCL22 axis is shown to be crucial in Treg recruitment to tumors, enhancing VEGF levels followed by angiogenesis and also cancer metastasis [95, 96]. CCR5 [97], CCR10 [98] and CXCR3 [99] are some of the other chemokine receptors shown to be involved in 
Treg recruitment to tumors. MDSC myeloid cell subset was shown to secrete chemokines like CCL3, CCL4, and CCL5 that lead to CCR5 mediated recruitment of Treg cells to tumors [97]. Apart from CCR5 chemokines, CCR2CCL2, CXCR2-CXCL5 and CXCR4-CXCL12 axis have been shown to promote tumor progression by enhancing the suppressive activity of TAMs and MDSCs [81]

\section{Chemoattractants recruit activated $\mathrm{CD}^{+} \mathrm{T}$ cells to target tissues}

The mechanisms of chemoattractant-regulated CTL migration to target tissues have been extensively investigated in the context of allergy, inflammation, autoimmune and infectious diseases, as well as in transplantation. However, limited information is available on CTL migration into tumors. Perhaps the knowledge on CTL migration in other diseases might guide further studies in the context of CTL migration to tumors.

In preclinical models of allergic inflammation and airway hyper-responsiveness, the $\mathrm{LTB}_{4}-\mathrm{BLT} 1$ axis plays a critical role in controlling the migration of activated CTLs into the airway [100]. The induction of BLT1 in CTLs and BLT1 dependent chemotaxis in effector but not in naive or central memory cells was demonstrated. In addition to BLT1, chemokine receptors CCR5, CXCR3, CXCR6 have all been implicated in CTL migration to lungs under pathophysiological conditions [101-103]. The role of CCR2 on CTLs in autoimmune systemic lupus erythematosus is well recognized; CCR2-deficient CTLs are unable to migrate to the kidney, thus reducing the score of autoimmune disease [104]. CCR9 and its 
ligand CCL25 control CTL migration, including to the small intestine, during inflammatory bowel disease [101]. In patients with rheumatoid arthritis, the CX3CR1: CX3CL1 axis is involved in the recruitment of autoimmune CTLs to the synovium [105]. Migration of CTLs in CXCR6, CCR4 and CCR10 dependent fashion to inflamed skin in psoriasis is also well established [106]. Myelin specific CTLs migrate to multi pre-sclerotic lesions in a CXCR3 and CCR5 dependent manner [107]. Thus, a wide range of chemokines and their receptors appear to control CTL migration in a target tissue-dependent manner. This could have implications for CTL migration to tumors in different anatomical locations.

An important physiological function of CTLs is to combat infections. The chemokine receptor CXCR3 and its ligands CXCL9/10 have been shown to mediate the recruitment of CTLs in a variety of infectious diseases [108, 109]. CXCL10 was recently shown to enhance CTL-mediated control of the pathogen Toxoplasma gondii in the brains of the chronically infected mice [110]. In a model of HCV and HSV-2 infections, CXCR3 and its cognate ligands mediated CTL infiltration to the inflamed liver [108] and vaginal mucosa [109], respectively. Chemoattractant mediated CTL migration is also an important part of the pathology of post-transplantation complications. It is well known that $\mathrm{CCR}^{+}$and $\mathrm{CXCR}^{+} \mathrm{CTLs}$ are involved in cardiac allograft vasculopathy and acute allograft rejection [111, 112]. In chronic rejection of lungs, CCL5, CXCL9, CXCL10, CXCL11 and CCL2 were upregulated, which might contribute to the posttransplantation complications by recruitment of antigen-specific CTLs to the graft [101]. In graft versus host disease-induced hepatitis, CTL infiltration to the liver 
was mainly controlled by CXCR6 [113]. Hence, chemokines/ chemokine receptors are an integral element of CTL recruitment and present an attractive pharmacological target for intervention in various disease pathologies.

Recent advances in the imaging techniques, such as intravital microscopy, have allowed in vivo tracking of CTLs in real time to gain some insights on CTL migration to tumors. It has been demonstrated that recognition of cognate antigen by CTLs within tumors is a critical determinant of optimal CTL infiltration and killing of tumor cell [114]. Furthermore, CD44 dependent CTL migration within the tumor microenvironment was found to be an essential immunologic checkpoint that determines the potency of T-cell effector functions [115]. Boissonnas et al. showed that activated CTLs migrate at high instantaneous velocities in the periphery, but get arrested when in close contact with tumor cells expressing their cognate antigen; therefore, antigen expression by tumor cells determines both $\mathrm{CD}^{+} \mathrm{T}$ cell motility within the tumor and extent of tumor infiltration [116]. In this regard, Deguine et al. demonstrated a sharp contrast between the effector function of CTL and NK cells. Although NK cell formed dynamic contacts with tumor cells, CTLs formed stable contacts with tumor cells expressing their cognate antigen for exerting their cytotoxic functions [117]. Dense matrix was shown to resist the CTL migration because aligned fibers in perivascular regions and around tumor epithelial cell regions dictated the migratory trajectory of $\mathrm{T}$ cells and restricted them from entering tumor islets [118]. 


\section{Chemokine receptors controlling activated CTL migration to tumors}

Several studies with human cancers showed a strong correlation between CD8 ${ }^{+}$ T cell infiltration and long-term survival [119]. The newly activated CTLs must infiltrate the tumor to exert their cytotoxic effects for controlling tumor growth [40]. Among the many factors controlling CTL migration, chemoattractants play a pivotal role in shaping the intratumoral infiltration of activated CTLs. The release of individual chemokines, such as CCL3, CCL5, CCL20, CXCL10, CXCL16 and CX3CL1 at the tumor site was shown to enhance CTL recruitment and antitumor immunity, confirming their important role in this process [40]. Likewise, the chemokine receptors known to be crucial in $\mathrm{CD}^{+} \mathrm{T}$ cell recruitment to tumors till date are CX3CR1, CXCR6, CCR5 and CXCR3. Because of it's relevance in this thesis, CXCR3 will be described in detail. We have reviewed all these chemokine receptors in detail here [40].

\section{CX3CR1:}

The CX3CL1/CX3CR1 axis has been associated with high numbers of infiltrating CTLs and better prognosis in colorectal cancers [120]. CX3CL1 (fractalkine) gene transfer in tumor cells also showed marked antitumor activity primarily by enhancing the infiltration of T and NK cells in various cancers, [40, 121-123], as also DC maturation and activation in the tumors [124].

\section{CXCR6:}

Another relatively new player in this context is CXCL16, which was produced by tumor cells in response to ionizing radiation and the intratumoral CXCL16 
enhanced the recruitment of CXCR6- positive CTLs and thus anti-tumor immunity $[40,125,126]$

\section{CCR5}

CCR5 was the first chemokine receptor that was shown to be an important regulator of CTL trafficking to tumors because its cognate ligand facilitated the infiltration of CTL into tumors [127]. Intratumoral injection of a chimeric CCL5-lg encoding DNA plasmid was associated with the infiltration of increased numbers of $\mathrm{NK}, \mathrm{CD}^{+}$and $\mathrm{CD}^{+} \mathrm{T}$ cells and generation of effective antitumor immunity. This effect was lost in RAG-2 or CCR5-deficient mice, indicating that CCR5 dependent CTL migration was required for this antitumor effect [128]. Adenoviral gene transfer to induce CCL3 expression in B16-ova tumors in vivo increased the efficacy of adoptively transferred tumor-specific effector OT1 T cells expressing its cognate receptor CCR5 $[93,129]$. However, in human cancers, the role of CCR5 and its cognate ligands has been elusive because of the diverse observations in various types of cancers. For example, in lung cancer, CCL5 is associated with favorable disease prognosis [130] but in breast, cervical and colon cancers, CCL5 has been associated with adverse prognosis [131, 132]. In case of colon cancer, tumor-derived CCL5 has been demonstrated to recruit regulatory cells and enhance the ability of these cells to induce apoptosis in CTLs within the tumor microenvironment 


\section{CXCR3}

It has long been recognized that CXCR3 is an important chemokine receptor for controlling $\mathrm{CD}^{+} \mathrm{T}$ cell migration under diverse pathophysiological conditions, including tumors. Expressed on antigen-activated CTLs, this receptor recognizes three cognate ligands, which are interferon-inducible proteins; CXCL9, CXCL10 and CXCL11, produced by stromal cells including endothelial, epithelial and tumor cells in response to IFNY. CXCR3 has been extensively studied in autoimmune and viral diseases and recently in cancer. CXCR3 gene Cxcr3 is located on the sex-chromosome and is an X-linked gene both in mice and humans [133]. Since certain immune disorders like systemic lupus erythematosus (SLE) have increased incidence in females compared to males, there may be a possible CXCR3 dependent gender predisposition to certain immune disorders. Hyun et.al. have reported a possible correlation of c.12+234G $>$ A polymorphism in CXCR3 with asthma development especially in males [134]. Another study reported an association of the CXCR3 polymorphism rs34334103 with male patients with SLE and pleuritis development in those patients [135].

There may however In SP2/0 myeloma tumor model, CXCL10 expression via adenoviral gene transfer in combination with adoptive T-cell therapy completely eradicated the tumors, whereas both the adoptive T-cell therapy and CXCL10 adenoviral gene transfer treatments had minimal to no beneficial efficacy [136]. These results highlight the importance of the ligand (CXCL10) in tumors and the receptor (CXCR3) on the adoptively transferred CTLs in generating effective 
antitumor immunity. In the case of human renal cell carcinoma, intratumoral expression of chemokines CXCL9 and CXCL10 showed positive correlation with CTL infiltration and inverse correlation with tumor size. Moreover, it was also observed that tumors that expressed CXCL9/10 rarely recur after surgery, reinforcing the role of these pathways in antitumor immunity [129]. In a retrospective evaluation of melanoma tumors isolates, expression of CXCR3 by human CTLs was significantly associated with enhanced survival in stage III patients [137]. Human melanoma cell lines have been shown to secrete CXCR3 ligands in response to IFNy [138]. Analysis of the chemokines and their receptor expression using immunohistochemistry and flow cytometry in situ in colorectal patient's samples revealed pre- dominant IFNy positive CTLs co-expressing CCR5 and CXCR3 receptors [139]. In human hepatocellular carcinoma (HCC), it was shown that CXCR3 expression correlated with activation markers, such as CD69, and it was suggested to be an important receptor of CTL migration [140]. Conversely, another study on HCC showed functional desensitization of CXCR3 in lymphocytes, including CTLs from HCC patients, by CXCL10 secreted by tumor cells suggesting a new mechanism in HCC to induce dysfunction of active CTL migration and subsequently impaired immune defense against the tumor [141]. These studies emphasize the importance of the CXCR3-CXCL9/10 axis in generating antitumor immunity in mouse models and in multiple human tumors. Mikuchi et.al. recently elegantly demonstrated CXCR3 mediated signalling to be a critical and an indispensable checkpoint for tumor antigen specific $C D 8^{+} \mathrm{T}$ cells to traffic across the tumor vasculature for carrying out effective tumoricidal 
activity in mice and human melanoma [142]. CTL chemokine receptors CCR5 and CCR2 were not essential for CXCR3 mediated CTL extravasation across tumor vessels despite the presence of CCL2 and CCL5 chemokines in the tumor mileu. A recent study demonstrated that adenosine in the tumor milieu suppressed the production of CXCL10 followed by suppression in $\mathrm{T}$ cell infiltration; and partial reversion was seen upon adenosine receptor blockade [143]. 


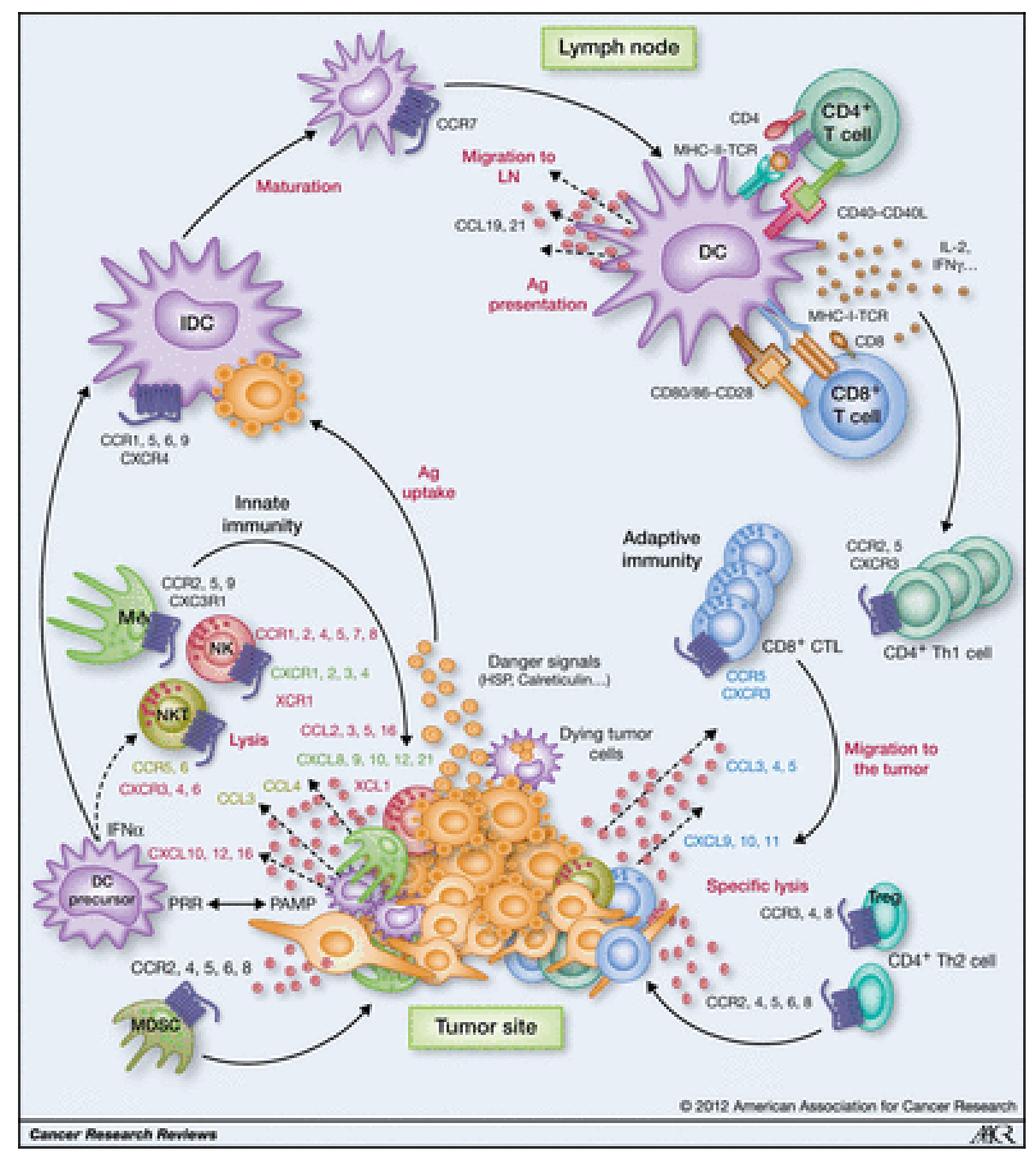

Figure 2: Chemokine network in the anti-tumor immune response. Malignant cells express pathogen-associated molecular patterns (PAMP) that can be recognized by pattern recognition receptors (PRR) on dendritic cells (DC) and macrophages (M), triggering release of chemokines.This results in recruitment and activation of $\mathrm{M}, \mathrm{NK}$, and NKT cells, which are able to lyse tumor cells. DC phagocytoses apoptotic tumor cells and HSP-complexed tumor-derived peptides. Upon maturation, DC change their homing proprieties by downregulating tissuespecific chemokine receptors and upregulating CCR7 that guides them to 
CCL19/CCL21-rich lymph nodes (LN), where they present processed tumor peptides to $\mathrm{CD}^{+}$and $\mathrm{CD}^{+} \mathrm{T}$ cells. Activated $\mathrm{T}$ cells upregulate expression of chemokine receptors including CCR5 and CXCR3, and in response to intratumoral chemokines, circulating CTL infiltrate the tumor to destroy malignant cells

Figure adapted from [144]. 


\section{Chemoattractants \& their receptors as targets for enhancing CTL}

recruitment

Poor CTL trafficking to tumors has become the rate-limiting step in the success of immunotherapies as suggested by evidence from clinical trials [40, 75, 76, $145,146]$. Recently, there have been studies showing modulation of chemokine or chemokine receptor expression to enhance CTL migration to tumors $[77,122$, 147-149]. Activation of Toll-like receptors 3 on mast cells through Poly-ICinduced CXCL10 and RANTES production leads to an increase in CTL recruitment [150]. IL-12 treatment of a fibrosarcoma and ovarian cell-derived tumor resulted in tumor regression via CCR5 dependent CTL migration [151]. lonizing radiation enhanced the recruitment of $\mathrm{CXCR}^{+} \mathrm{CTLs}$ and antitumor response in 4T1 breast cancer mouse model [125]. In a recent study, combining radiotherapy and ipilimumab resulted in abscopal effects in a treatment-refractory lung cancer patient that correlated with enhanced CTL infiltration [152]. Chemotherapy, such as low-dose cyclophosphamide and gemcitabine, can eliminate the T regulatory cells [153] and MDSCs [154] respectively, allows for the production of immunostimulatory antitumor cytokines, such as IFNY and TNF$\alpha$, which can induce the CXCR3 and CCR5 mediated feed-forward recovery of CTL recruitment. Radiation therapy, along with its direct cytotoxic effect, also has an immunomodulatory effect through interferon-induced chemokine CXCL10 production by the myeloid cells in tumors. CXCL10 increases CXCR3 mediated recruitment of CTLs into the tumors for this immunomodulatory effect [155]. 
Direct modulation of CCL5 in the tumor environment was found to enhance CTL infiltration and tumor rejection in an immunogenic fibro- sarcoma genetically modified to express CCL5 in murine model [148].

Various CAR-based therapies using chemokine receptors have been used to enhance the recruitment of tumor specific CTLs into tumors. For instance, in a CAR-based ACT for mesothelioma, the infiltration of human T cells in tumors injected with untransduced $T$ cells or with the mesoCAR $T$ cells was very low $(\sim 0.3-0.4 \%)$ and not significantly different. In contrast, the infiltration of transferred mesoCAR + CCR2b gene modified T cell group was dramatically improved to $5.2 \%$ ( $>12.5$-fold) when compared with the mesoCAR or the untransduced T cell groups [77].

The Reed-Sternberg cells of Hodgkin lymphoma produce CCL17 and CCL22, recruiting $\mathrm{CCR}^{+} \mathrm{T}$ regulatory cells to tumor-causing immune suppression [156]. Modification of CTLs instead, to express both CAR directed to the Hodgkins lymphoma associated antigen CD30 and CCR4 resulted in >10-fold increase in tumor infiltration of CTLs by day 12. Infusion of these cells intravenously caused tumor regression in $57 \%$ of the mice [156]. These directed therapies enhanced the antitumor responses of the CAR CTLs and also increased the effective migration of these cells into the tumors.

CTL migration to tumors was enhanced by transfecting the CTLs with photoactivable chemokine receptor (PA-CXCR4) that induced their homing to tumors when tumors were exposed to light at $505 \mathrm{~nm}$ [157]. This study proposes 
the use of photoactivatable chemokine receptors as an important tool to enhance CTL recruitment, particularly in CTL based therapies, such as adoptive $T$ cell transfer therapies [157]. All of these studies demonstrate the potential for targeting the chemokine/chemokine receptor directly or indirectly to enhance CTL cell migration into tumors and thus T cell mediated immunotherapies.

\section{Barriers to CTL infiltration to tumors}

The tumor evades the immune control by manipulating the immune system into supporting its growth, including restricting the $\mathrm{CD}^{+} \mathrm{T}$ cell infiltration into the tumor microenvironment $[40,158]$. Anti-tumor cytokines like IFNy have shown to be crucial for T cell homing to tumors [159]. However, the tumor mileu is largely immune-suppressive instead due to expression of molecules like TGF- $\beta$, IL-10, IDO, VEGF-A, adenosine, etc. that compromise T cell recruitment process [75]. Mechanisms by which T cell homing to tumors gets abrogated is discussed below.

The immune-suppressive environment not only hinders T cell homing to tumors, but those that enter are restricted at the tumor margins or periphery. Studies in breast cancer model showed that tumor dendritic cells (Tu-DCs) and tumor associated macrophages (TAMs) constitutively cross present the tumor antigens to the infiltrating tumor specific $T$ cells engaging them with long-unproductive interactions at the tumor margin [160]. Another study demonstrated that adoptively transferred $T$ cells were trapped in TuDC-mesh like structures that prevented the homing of T cells deeply in the tumor beds [161]. 
Cytotoxic cell to tumor cell ratio is critical for anti-tumor immunity. T cells are present in higher numbers in tumors that express chemokines like CXCL9, CXCL10, CCL2, CCL3, CCL4 and CCL5 [162]. Tumors usually alter the chemokine profile thus disturbing the balance between $\mathrm{T}$ cells and tumors. Tumor mileu can potentially induce chemical modification of chemokines thereby rendering them non-functional for CTL recruitment. For example, nitrosylation of CCL2 preferentially recruits MDSCs and not tumor specific T cells [163]. Likewise tumors under hypoxia also produce CCL28 that preferentially recruit the Tregs [98]. Aberrant EGFR-Ras signaling in skin tumors was shown to suppress the production of CCL27 chemokine by keratinocytes and absence of CCL27 was shown to prevent $T$ cell infiltration to tumors and anti-tumor immunity [164]. Another mechanism for preventing CTL infiltration was postulated to be due to altered proteolytic processing of the CXCR3 chemokine CXCL11 [165]. Thus tumor strategizes immune evasion in various ways including alteration of chemokine production to prevent $\mathrm{CD} 8^{+} \mathrm{T}$ cell infiltration to tumors.

Yet another mechanism that prevents $T$ cell recruitment to tumors is the disorganized and aberrant nature of the tumor associated endothelial vessels. Growth factors in the tumor mileu like endothelin 1, basic fibroblast growth factor (bFGF) and VEGF-A blocks/downregulates the expression of ICAM1 and VCAM1; a phenomenon called "Endothelial cell anergy". This leads to attenuation of $\mathrm{T}$ cell adhesion to tumor endothelium and thereby prevents their infiltration into tumors $[75,166]$. Although the tumor endothelium is considered as leaky, the tumors with enhanced angiogenesis typically lack T cells. In an ovarian 
cancer study, it was reported that VEGF expression was three folds more in tumors without $T$ cells than with $T$ cells [167]. Angiogenesis in the context of tumor leads to the generation of new tumor vessels that are quite irregular compared to the normal vessels. The tumor vessels that originate from the existing ones in the tumors are leaky, disorganized, dilated with abnormal structural morphology, absent or loosely attached pericytes and unusually thick basement membrane $[146,168]$. This abnormal nature of the tumor vasculature may increase the interstitial pressure and promote aberrant blood flow making it difficult for selective leukocytes to adhere and traffic into the tumor mass even after being activated in the periphery.

Absence of certain chemokine receptors can seriously compromise $\mathrm{T}$ cell homing to tumors. Importance of chemokine-chemokine receptor systems in regulating $\mathrm{CD}^{+} \mathrm{T}$ cell homing to tumors is only recently being acknowledged. Apart from the chemokine receptors discussed in the previous section there are no other known chemokine-chemokine receptor systems that are known to be crucial for $\mathrm{CD}^{+} \mathrm{T}$ cell recruitment into tumors and hence necessitates research in this area. Targeting chemokine-chemokine receptor pathways can enhance $\mathrm{T}$ cell homing to tumors. Recent studies have indicated the presence of tumor specific $T$ cells in the tumors for the immunotherapies to be successful. In fact the data presented in this thesis indicate that PD-1 blockade based immunotherapy completely fails in $\mathrm{BLT}^{-/-}$and $\mathrm{CXCR}^{-/}$mice suggesting the importance of chemokine receptors and $\mathrm{T}$ cell homing for the success of immune checkpoint blockade based therapies. 


\section{Leukotriene $B_{4}$ and its high affinity receptor BLT1}

Leukotriene $\mathrm{B}_{4}(5(\mathrm{~S}), 12(\mathrm{R})$-dihydroxy-6, 14-cis-8, 10-trans-eicosatetraenoic acid, $\mathrm{LTB}_{4}$ ) is a potent lipid inflammatory mediator, a calcium ionophore, that causes adhesion and chemotactic movement in leukocytes and stimulates aggregation, enzyme release and superoxide generation in neutrophils $[169,170]$. LTB ${ }_{4}$ is derived from arachidonic acid released from membrane phospholipids via the actions of phospholipase A2. Enzymes 5-lipoxygenase (5-LO) [171] and LTA 4 hydrolase [172] catalyze the production of $\mathrm{LTB}_{4}$ from arachidonic acid in a sequential manner. While $\mathrm{LTA}_{4}$ hydrolase is universally present in all cells, 5lipoxygenase is only expressed in hematopoietic cells mostly myeloid cells. LTB 4 is produced by myeloid cells mostly, however, under inflammatory condition, $\mathrm{LTB}_{4}$ production in other cells (non-immune cells) have also been reported via transcellular transport of $\mathrm{LTA}_{4}$ from immune cells at the site of inflammation to other cells that have $\mathrm{LTA}_{4}$ hydrolase activity [173]. $\mathrm{LTB}_{4}$ signals through two $\mathrm{G}$ protein coupled seven-transmembrane domain receptors, BLT1 and BLT2, the high and low affinity receptors, respectively [174-176]. BLT1 receptor activation upon ligand binding leads to IP3 mediated calcium release from intracellular portions and later calcium influx through the cell membrane. In general, BLT1 receptor enhances the production of inositol phosphates, mobilize intracellular calcium, and activate several kinases, including PI3K, MAPK and AKT [177]. BLT1 is expressed on a variety of immune cell subsets including neutrophils, eosinophils, monocytes, dendritic cells, and activated T cells [178]. The role of 
BLT1 as a pro-inflammatory mediator was first known when BLT1 deficient mice showed significantly reduced arachidonic-acid induced ear inflammation [179].

The protective phenotype observed in BLT1 knockout mice in various inflammatory disease models like asthma, atherosclerosis, arthritis, autoimmune uveitis, and diet-induced obesity later on indicated that BLT1 mostly acts as a pro-inflammatory mediator [180-184]. However, BLT1 mediated migration of different cell types were the causative reasons behind each of these diseases. For example, BLT1 mediated recruitment of neutrophils was crucial for arthritis to develop; macrophage recruitment via BLT1 was crucial in atherosclerosis and diet induced obesity manifestation; and BLT1 mediated recruitment of activated T cells in the development of autoimmune uveitis and airway-hyperresponsiveness.

The biological significance of this receptor in antitumor immunity has not been explored. The majority of work carried out in the context of inflammatory, autoimmune diseases demonstrated that $\mathrm{CD}^{+} \mathrm{T}$ cells inducibly express BLT1 upon activation and the receptor expression is essential for their recruitment to target organs and disease development [100]. In a model of autoreactive T cellinduced uveitis, BLT1 expression on both T cells and innate immune cells was found critical for full disease development, and absence of BLT1 is highly protective in ocular inflammation [183]. However, in the context of cancer, the lack or delay in recruitment of effector immune cells such as T cells may delay generation of immune response to tumor $\mathrm{Ag}$ that can lead to breach of immune surveillance and poor antitumor immunity. 
To determine the role of BLT1 in anti-tumor immunity, implantable model of TC-1 cervical cancer was employed and the data from these experiments is briefly described and formed the basis for the experiments conducted in the current thesis. The results obtained after challenging WT and BLT1 deficient mice with TC-1 cervical cancer is discussed in the next chapter. 


\section{CHAPTER II}

\section{LEUKOTRIENE B 4 RECEPTOR, BLT1 REGULATES ANTI-TUMOR IMMUNITY BY MEDIATING CD8 ${ }^{+}$T CELL INFILTRATION TO TUMORS}

BLT1 the high affinity receptor for Leukotriene $B_{4}$ has long been identified as a pro-inflammatory mediator in various disease models of infection, auto-immunity, inflammation and tumors. BLT1 is expressed on a variety of immune cells as discussed before. Migration of specific cell types is crucial in determining the type of inflammation (pro or anti-tumor growth) that ensues. Infiltration of $\mathrm{CD}^{+} \mathrm{T}$ cells into the tumors is a critical event for effective antitumor immunity.

The results presented in this section details the role of $B L T 1$ in regulating $C D 8^{+} T$ cell infiltration to tumors and anti-tumor immunity using a viral antigen based subcutaneous implantable TC-1 cervical cancer model [176]. Implantation of $2 \times 10^{4} \mathrm{TC}-1$ cells resulted in development of tumors only in $50 \%$ of WT mice (sublethal dose). However, under these conditions, $100 \%$ of $\mathrm{BLT}^{-{ }^{--}}$mice developed tumors. At this dose, while $100 \%$ of $\mathrm{BLT}^{-/}$mice succumbed to disease by day $50,60 \%$ of WT mice still survived at day 80 -post tumor challenge (Figure $3 \mathrm{~A}$ ). Subcutaneous implantation of $10^{5}$ TC-1 cells (lethal dose) also resulted in significantly enhanced tumor growth and reduced survival in $\mathrm{BLT1} 1^{-/}$mice compared to WT mice (Figure $3 \mathbf{B}$ and $\mathbf{C}$ ). These results indicated that BLT1 deficient mice have poor immune surveillance against TC-1 tumors. 
Development of cancer is proposed to be the end result of a malignant transformation that has passed through all three phases viz. elimination, equilibrium, and escape phases [18]. The data obtained with lethal and sub-lethal doses of tumor cells suggest a crucial function for BLT1 in controlling both elimination and equilibrium phases of tumor development. The sub-lethal dose of tumor led to slow growing tumor formation in only $50 \%$ of WT mice but showed tumor development in $100 \%$ of the BLT1 deficient mice suggesting that BLT1 is a crucial component of immune surveillance to tumors. The lethal dose of tumor cells led to rapidly growing tumors in $\mathrm{BLT}^{-/}$mice, indicating its function in antitumor immunity [176].

To determine the cellular mechanisms behind this phenotype, tumor immune infiltration studies were carried out. A marked decrease in infiltration of overall CD45.2 $2^{+}$immune cells with most striking decline in $\mathrm{CD}^{+} \mathrm{T}$ and $\mathrm{NK}$ cell population in tumors of $\mathrm{BLT1}^{-/-}$mice compared with WT (Figure 4A - 4C) was observed. To ensure that this difference was not a function of enhanced tumor size in $\mathrm{BLT}^{-/}$mice; $\mathrm{CD}^{+} \mathrm{T}$ cells at similar tumor sizes were stained that also showed significant reduction of $\mathrm{CD} 8^{+} \mathrm{T}$ cells in tumors of $\mathrm{BLT} 1^{-1-}$ mice compared to WT mice (Figure 4D). Also no significant difference in $\mathrm{CD}^{+} \mathrm{T}$ cell numbers was observed in spleen and tumor draining lymph nodes of WT and $\mathrm{BLT1}{ }^{-/-}$mice (Figure 4E and 2F) suggesting that $\mathrm{BLT1}$ deficient $\mathrm{CD}^{+} \mathrm{T}$ cells showed defective tumor infiltration ability. Gene expression analysis by RT-PCR showed a significant reduction in effector T cell transcript levels such as IFN- $y$, granzyme $\mathrm{B}$, and IL-2 in tumors of $\mathrm{BLT1}^{-/-}$mice relative to WT mice (Figure 4G). 
To understand whether BLT1 expression on $\mathrm{CD}^{+} \mathrm{T}$ cells or NK cells is crucial in the observed phenotype; $\mathrm{BLT} 1^{-/} \mathrm{RAG}^{-/-}$mice were generated by crossing $\mathrm{BLT} 1^{-/-}$ mice with Rag2 ${ }^{--}$mice. Comparison of tumor growth kinetics in Rag2 ${ }^{-/}$and $\mathrm{BLT1}^{-}$ ${ }^{/}$Rag2 ${ }^{-/}$mice revealed no significant differences in tumor growth indicating that BLT1 expression on innate cells including NK cells does not play a dominant role in this model. Also, similar levels of NK cells were seen in tumors of $\mathrm{Rag2}^{-/-}$and $\mathrm{BLT1}^{-/}{ }^{-1}$ ag2 $2^{-/}$mice (Figure 5A and 5B) [176]. Although NK cells by themselves may not hinder tumor growth in this model, it is still possible through CD8-NK cross talk that they contribute to effective antitumor immunity [185].

To further understand the importance of $\mathrm{BLT} 1$ on $\mathrm{CD} 8^{+} \mathrm{T}$ cells, $\mathrm{CD} 8^{+} \mathrm{T}$ cells were depleted in WT and BLT1 ${ }^{-1-}$ mice following TC-1 tumor challenge using CD8 depleting antibody (Bioxcell). Depletion of $\mathrm{CD} 8^{+} \mathrm{T}$ cells resulted in a significant acceleration of tumor growth only in WT and not in $\mathrm{BLT1}^{-/-}$mice. Moreover, the tumor growth in the CD8-depleted WT mice nearly overlapped with the tumor growth in $\mathrm{BLT1}^{-/-}$control mice and/or $\mathrm{CD} 8^{+} \mathrm{T}$ cell-depleted $\mathrm{BLT1} 1^{-/-}$mice (Figure 6). Therefore, elimination of $\mathrm{CD} 8^{+} \mathrm{T}$ cells alone was sufficient for complete loss of the observed phenotype, implicating a central role for BLT1 expression on $\mathrm{CD}^{+} \mathrm{T}$ cells.

Adoptive transfer of tumor experienced WT but not $\mathrm{BLT}^{-{ }^{-}} \mathrm{CD} 8^{+} \mathrm{T}$ cells intravenously could significantly reduce tumor growth in Rag2 $2^{-/-}$mice challenged with TC-1 tumors (Figure 7). This experiment served as a simplified replica of the adoptive $\mathrm{T}$ cell therapy used in the clinic following immune ablation. CTL infiltration studies suggested that the transferred $\mathrm{WT} \mathrm{CD}^{+} \mathrm{T}$ cells entered 
tumors in greater numbers compared to $\mathrm{BLT}^{-/-} \mathrm{CD}^{+} \mathrm{T}$ cells; their numbers being similar in the tumor draining lymph nodes (Figure 7B and 7C). Analysis of the major $\mathrm{CD}^{+} \mathrm{T}$ cell chemokine receptors, CCR5, CCR9, CXCR3 and BLT1 on the transferred $\mathrm{WT}$ and $\mathrm{BLT1}{ }^{-/}$CTLs in TdLN and tumor revealed no striking differences besides the significantly enhanced expression of BLT1 on WT CTLS in TDLNs compared to the absence of its expression in naive WT and BLT1 $1^{-1}$ CTLs (Figure 8). A significant increase in CXCR3 expression was observed in both adoptively transferred WT or $\mathrm{BLT}^{-1_{-}} \mathrm{CD} 8^{+} \mathrm{T}$ cells relative to the $\mathrm{CD} 8^{+} \mathrm{T}$ cells from the naïve mice. Analysis of expression of all these receptors on $\mathrm{CD} 8^{+} \mathrm{T}$ cells showed complete downregulation in tumors which is consistent with our previous study and many others suggesting receptor internalization upon ligand binding upon target sites. 
A

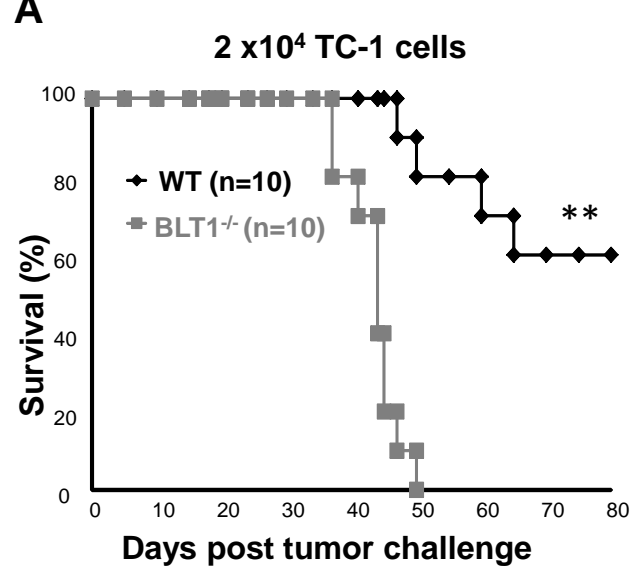

B

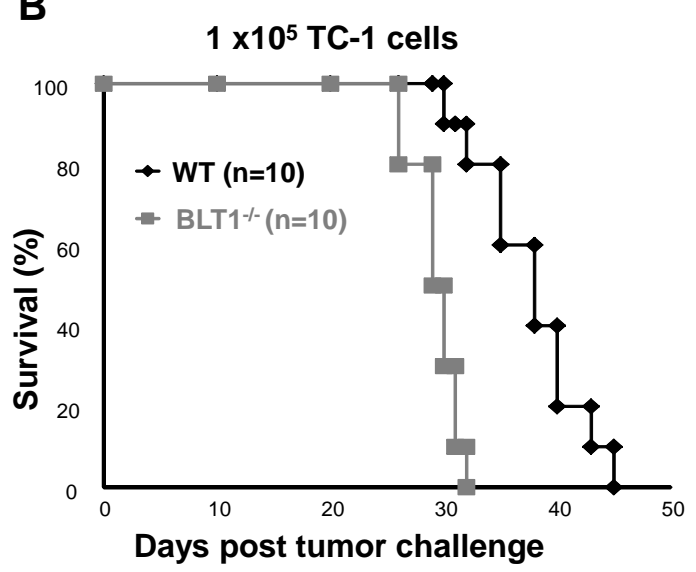

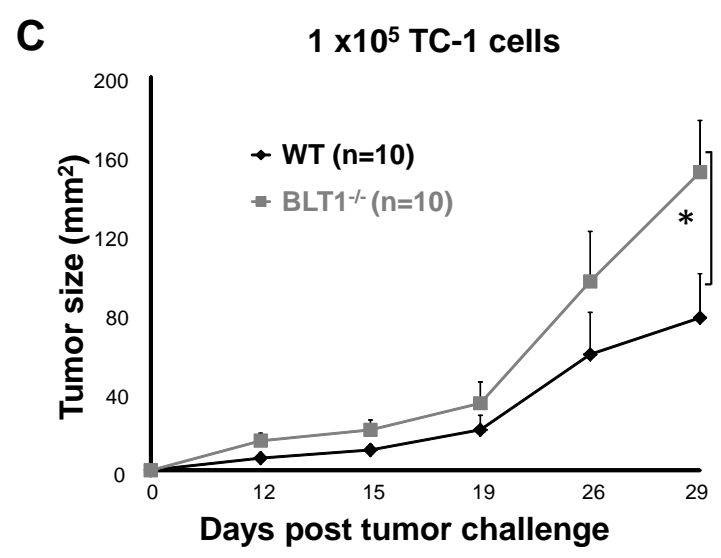

Figure 3: Decreased survival and increased tumor growth in BLT1 ${ }^{-/-}$mice. (A and B) Significantly reduced survival of $\mathrm{BLT}^{-/-}$mice using sub-lethal and lethal TC-1 dose. Kaplan-Meier survival plots of $\mathrm{BLT}^{+/+}$and $\mathrm{BLT1} 1^{-/-}$mice. (A) The mice were injected s.c. with a sublethal dose $\left(2.0 \times 10^{4}\right)$ of TC-1 cells and their survival was monitored up to $80 \mathrm{~d}$. (B) $\mathrm{BLT} 1^{+/+}$and $\mathrm{BLT1} 1^{-/-}$mice were injected s.c. with a lethal dose $\left(1.0 \times 10^{5}\right)$ of TC-1 cells and survival was followed. (C) Significantly enhanced tumor growth in $\mathrm{BLT}^{-/-}$mice. Tumor size in lethal dose challenged group was measured and calculated by multiplication of two perpendicular diameters (length $\mathrm{x}$ width). Log rank tests were performed for statistical analysis of survival, and student t test was used for tumor sizes. 
Experiment shown is representative of three independent experiments. ${ }^{*} p<0.05$ (significant), ${ }^{* *} \mathrm{p}<0.001$ (very significant). 
A

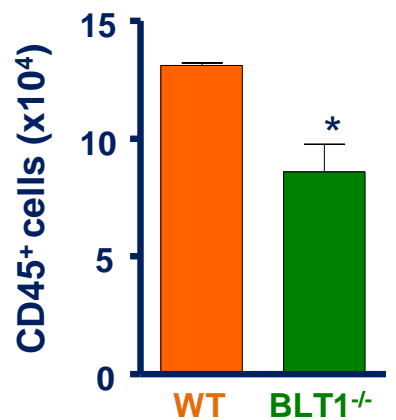

D

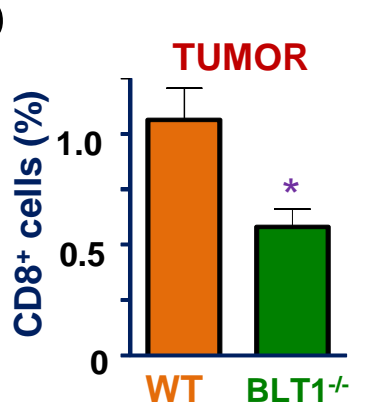

G

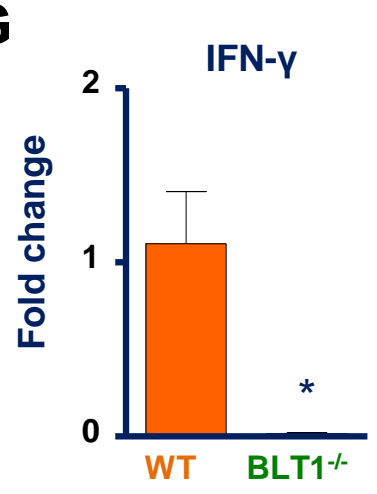

B

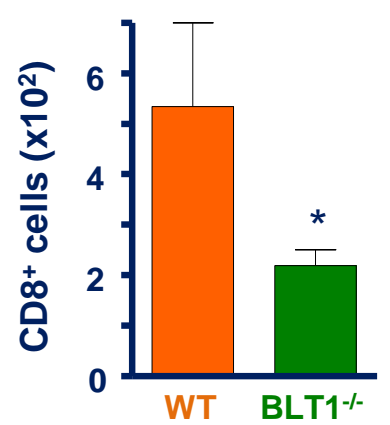

$\mathbf{E}$

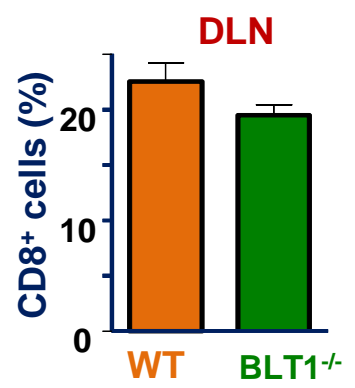

H

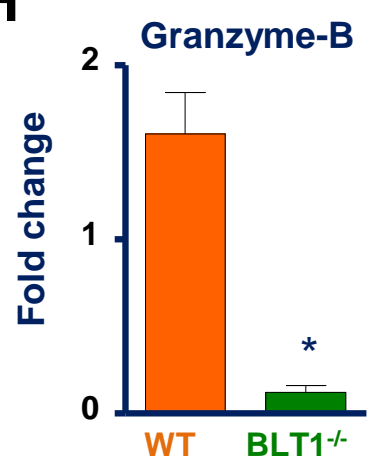

C

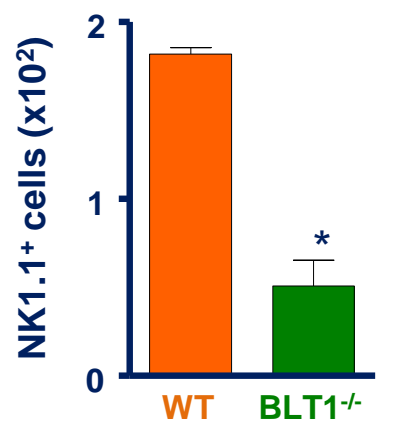

$\mathbf{F}$
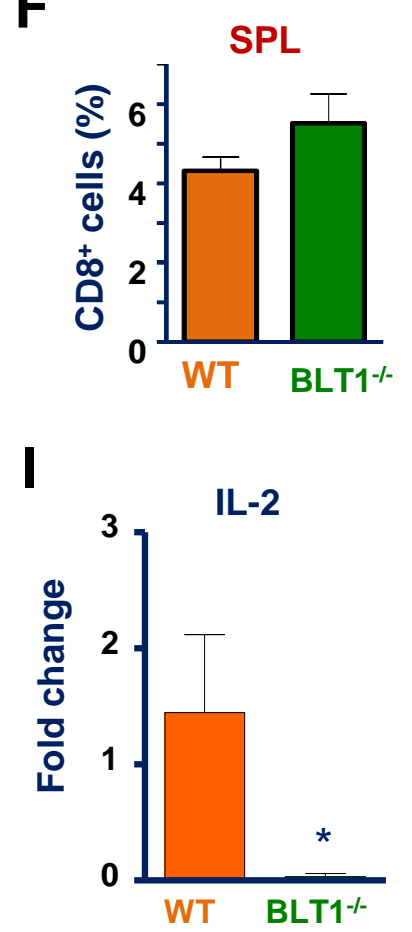

Figure 4: Reduced infiltration of effector antitumor immune cells into TC-1 tumors growing in $\mathrm{BLT1}^{-/-}$mice. $(\mathrm{A}-\mathrm{C}) \mathrm{BLT}^{+/+}$and $\mathrm{BLT1} 1^{-/}$mice were injected s.c. with $1.0 \times 10^{5} \mathrm{TC}-1$ cells, and tumors were harvested at $29 \mathrm{~d}$ of post tumor challenge. Numbers of total $\mathrm{CD} 45^{+}$immune cells (A), CD8 ${ }^{+} \mathrm{T}$ cells (B), and NK 
cells $(\mathrm{C})$ per million of total tumor cells were analyzed from WT and $\mathrm{BLT}^{-/-}$mice using standard flow cytometry methods. (D-F) $\mathrm{CD}^{+} \mathrm{T}$ cell staining in size matched tumors showing $\% \mathrm{CD}^{+} \mathrm{T}$ cells (frequency of total) in tumor (D), \% $\mathrm{CD}^{+} \mathrm{T}$ cells (frequency of $\mathrm{CD} 45^{+}$) in $\operatorname{TdLN}(\mathrm{E})$, and spleen (F) from WT and $\mathrm{BLT1}^{-/-}$mice. (G-I) Quantitative real-time PCR analysis: The levels of IFNy (G), granzyme B (H), and IL-2 (I) mRNA expression in BLT1/ tumors as compared with WT tumors by qRT-PCR were determined. Data are representative of two to three independent experiments involving at least $n=4$ mice/group in each experiment. ${ }^{*} p<0.05$ 
A

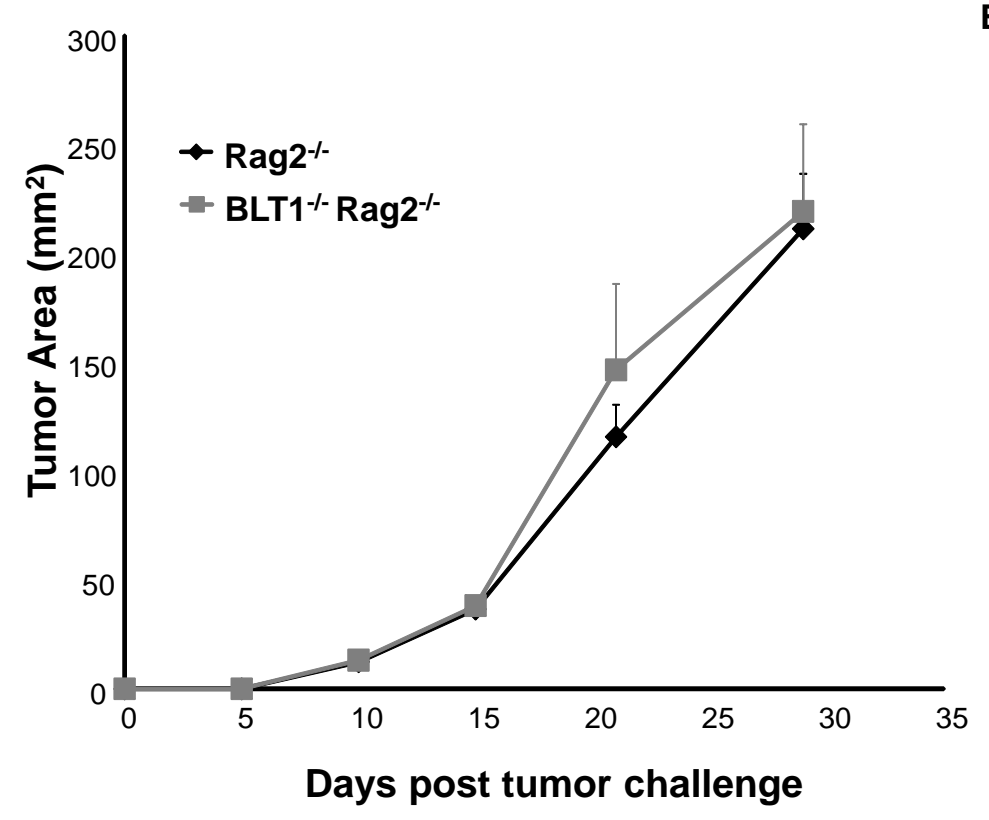

B

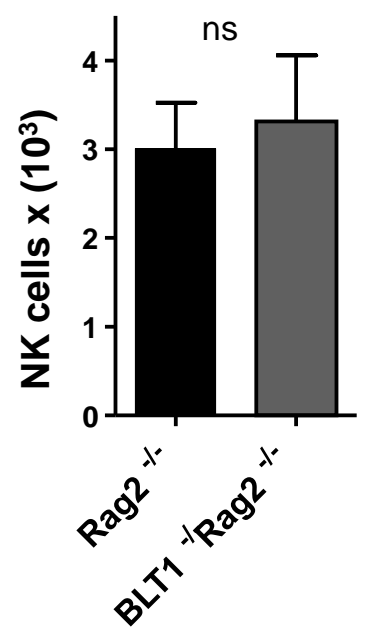

Figure 5: Unaltered tumor growth and intratumoral NK cell numbers in Rag2 $^{-/-}$and Rag2 $^{-/-}$BLT1 $^{-/-}$mice. (A) Rag2 $2^{-/-}$and Rag2 $^{-/-B L T 1} 1^{-/-}$mice were challenged s.c. with $5 \times 10^{4} \mathrm{TC}-1$ cells on the right flank and observed for the rate of tumor growth. (B) Absolute numbers of tumor-infiltrating NK cells per million of total tumor cells were analyzed from Rag2 ${ }^{-/-}$and $\operatorname{Rag}^{-/-} \mathrm{BLT}^{-/-}$mice using standard flow cytometry methods. Data shown are representative of three independent experiments. 


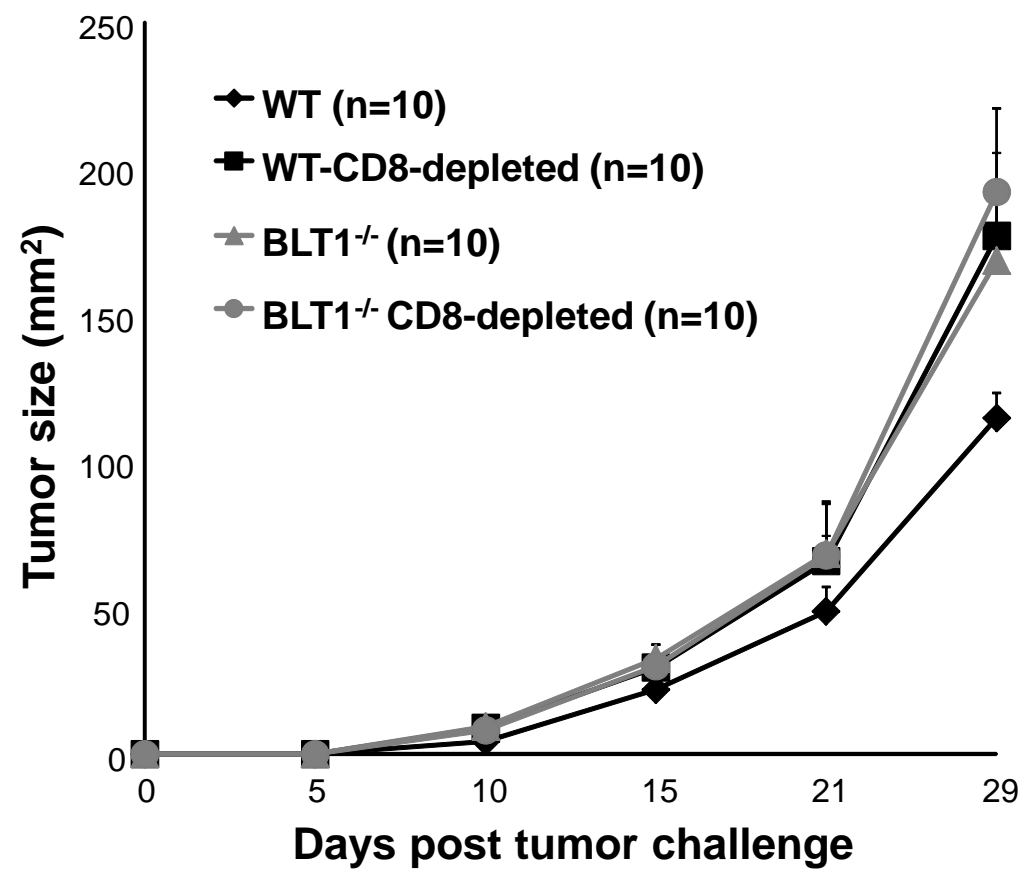

Figure 6: Depletion of $\mathrm{CD}^{+} \mathrm{T}$ cells accelerates TC-1 tumor growth in $\mathrm{BLT1}^{+/+}$but not in $\mathrm{BLT1}^{-/-}$mice. A single dose of $500 \mu \mathrm{g}$ CD8-depleting Ab was injected i.p. in WT and $\mathrm{BLT1}^{-/}$mice. The next day, $1 \times 10^{5} \mathrm{TC}-1$ tumor cells were inoculated S.C in the right flank in WT and $\mathrm{BLT} 1^{-/}$mice, and the tumor growth was monitored. Data shown are representative of three independent experiments. ${ }^{*} \mathrm{p}<0.05$ 


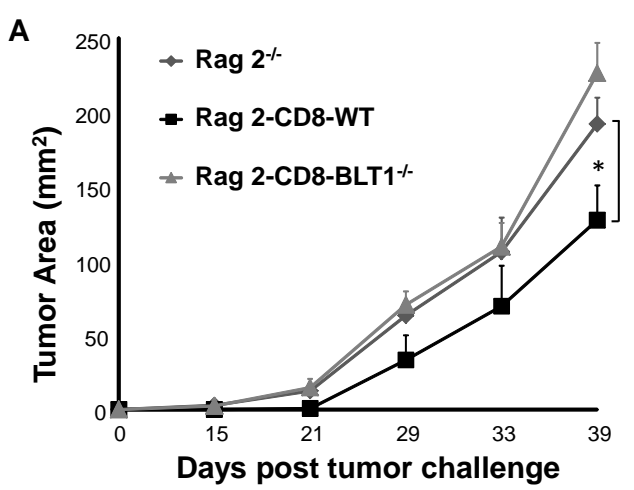

B

C
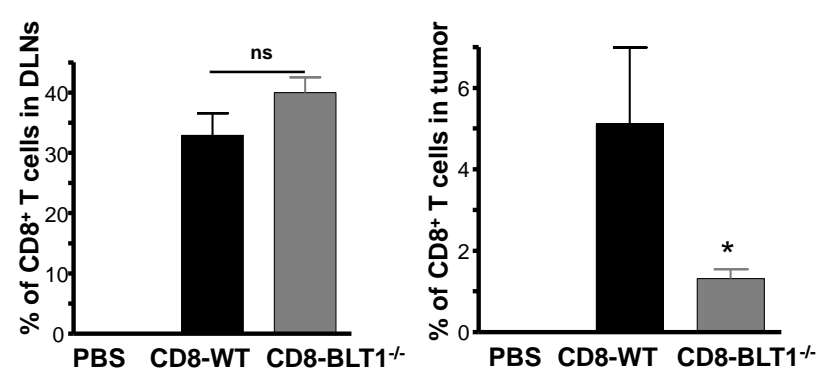

Figure 7: Adoptive transfer of tumor primed $\mathrm{CD}^{+} \mathrm{T}$ cells from $\mathrm{BLT1}^{+/+}$but not from $\mathrm{BLT1}^{-/-}$mice retards tumor growth. Rag2 ${ }^{-/}$mice were challenged with $5 \times 10^{4} \mathrm{TC}-1$ tumor cells in the right flank. Two days later, CD8 ${ }^{+} \mathrm{T}$ cells were isolated from the spleens and lymph nodes of tumor bearing (3-5 mm) WT or $\mathrm{BLT1}^{-/}$mice by magnetic sorting. A total of $8 \times 10^{5} \mathrm{CD} 8 \mathrm{~T}$ cells (97\% purity) or PBS was injected i.v. in the tumor inoculated Rag2 ${ }^{-/}$mice. (A) Tumor growth curve of Rag2 ${ }^{-/}$, Rag2 ${ }^{-/}$transferred either with $\mathrm{BLT}_{1}^{+/+}$or $\mathrm{BLT}^{-{ }^{--}} \mathrm{CD} 8^{+} \mathrm{T}$ cells. (B and C) The $\% \mathrm{CD}^{+} \mathrm{T}$ cells of total cells recovered from TDLNs (B) and $\% \mathrm{CD} 8^{+} \mathrm{T}$ cells of total $\mathrm{CD}_{4} 5^{+}$cells within tumors (C) are shown. Data shown are representative of three independent experiments $(n=5) .{ }^{*} p<0.05$ 


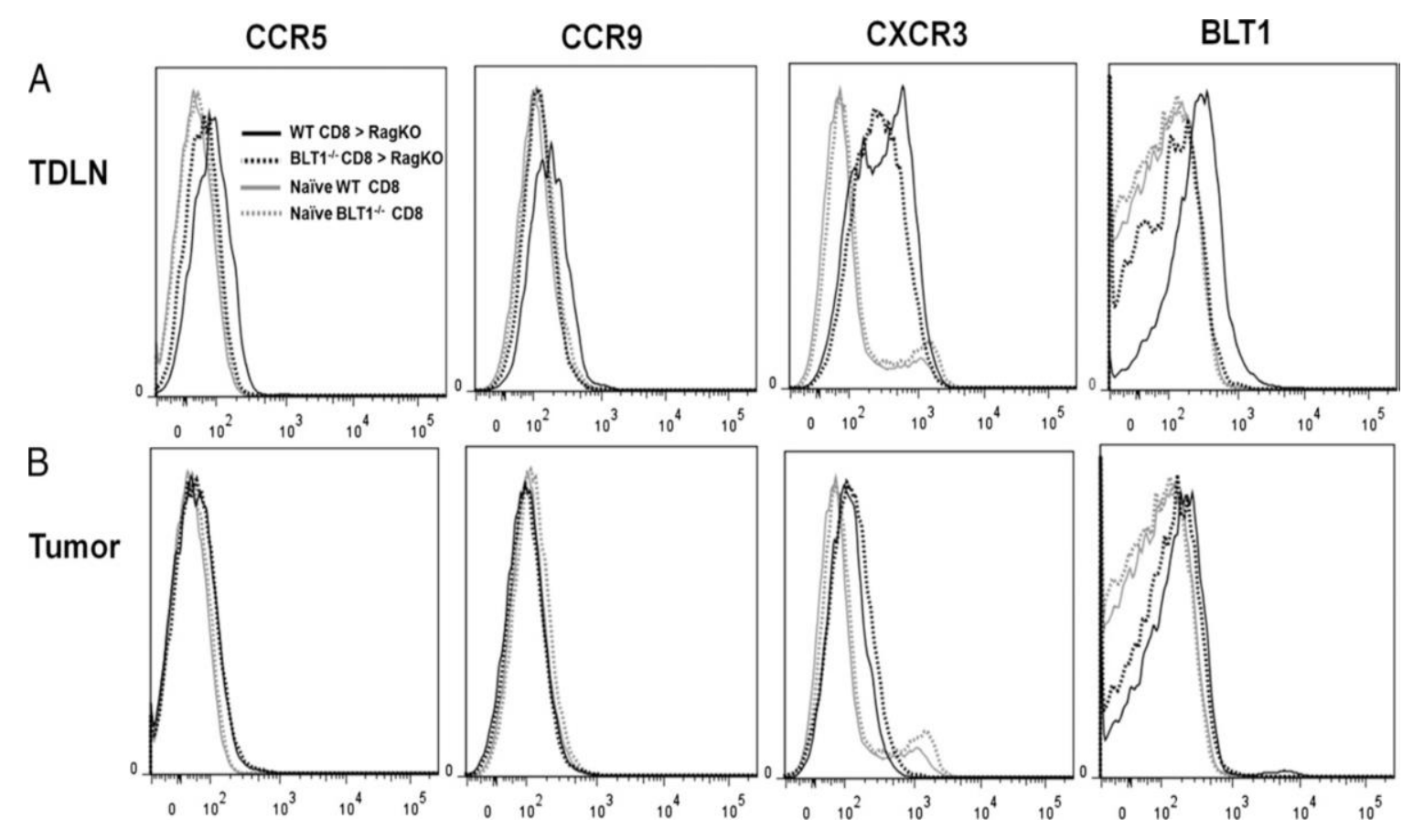

Figure 8: Chemokine receptor expression on the adoptively transferred $\mathrm{CD}^{+} \mathrm{T}$ cells from TDLNs and tumors. Adoptively transferred $\mathrm{CD} 8^{+} \mathrm{T}$ cells from WT and $\mathrm{BLT}^{-/-}$mice into Rag2 $2^{-/}$mice were analyzed for the expression levels of chemokine receptors. Naïve $\mathrm{WT}$ and $\mathrm{BLT} 1^{-/-} \mathrm{CD} 8^{+} \mathrm{T}$ cells were used as controls. Chemokine receptors CCR5, CCR9, CXCR3, and BLT1 were stained and analyzed on the transferred $\mathrm{CD} 8^{+} \mathrm{WT}$ or $\mathrm{BLT} 1^{--} \mathrm{CD} 8^{+} \mathrm{T}$ cells from TDLNs $(\mathrm{A})$ and tumor (B). Data shown are representative of two independent experiments. 
Taken together, using an implantable cervical cancer model, we demonstrated that expression of $\mathrm{BLT1}$ on $\mathrm{CD}^{+} \mathrm{T}$ cells plays a crucial role in mediating their recruitment to tumors, thereby initiating and sustaining antitumor immunity. In the context of cancer, BLT1 was shown to play a dual role in controlling tumor promoting inflammation; as seen in silica induced lung tumorigenesis [186] and or tumor suppressive inflammation; as shown in the TC-1 cervical cancer model [176]. A recent article by Yokota et al. used GM-CSF-based tumor vaccine setting in BALB/c leukemia model to evaluate the vaccine and secondary/recall immune responses. Their results showed similar or better primary and recall immune responses in the $\mathrm{BLT1}^{-/-}$mice [187]. Several differences that might account for the divergent results include: 1) different mouse strains (BALB/C), 2) different cancer type (leukemia), and 3) GM-CSF transformed cancer cell lines. In addition, Yokota et al.'s study found differences WT and BLT1 ${ }^{-/-}$mice only in the recall responses with $\mathrm{CD} 4^{+} \mathrm{T}$ cells playing a dominant role. In our studies, the numbers of tumor-infiltrating $\mathrm{CD} 4^{+} \mathrm{T}$ cells in $\mathrm{WT}$ and $\mathrm{BLT}^{-/-}$mice were similar, indicating a limited, if any, direct role of $\mathrm{CD}^{+} \mathrm{T}$ cells in controlling the tumor growth.

Hence, in context of cancers, BLT1 seems to have dual roles in tumor progression. Given the results on the role of BLT1 in CTL migration to tumors in viral antigen based cervical cancer model, the experiments conducted in this thesis are aimed at developing an understanding about the role of BLT1 in endogenous immune surveillance against other solid tumors, specifically nonviral, self-antigen based B16 melanoma model. We also studied the role of 
CXCR3 chemokine receptor in CTL migration to tumors and anti-tumor immunity and the possibility of interdependence of BLT1 and CXCR3 in achieving an antitumor immune response through the generation of BLT1/CXCR3 double knockout mice (DKO). The experiments also determine the importance of BLT1 and $\mathrm{CXCR} 3$ receptors in regulating PD-1 blockade based therapeutic vaccinate mediated anti-tumor effector responses. 


\section{CHAPTER III}

\section{MATERIALS AND METHODS}

\section{Mice and cell lines:}

C57BL/6 mice, $\mathrm{CXCR3}^{-1-}$ mice and UBC-GFP mice in C57BL/6 background (67wks old) were purchased from The Jackson Laboratory and/or bred in our animal facility at the University of Louisville. Previously described $\mathrm{BLT}^{-/}$mice in C57BL/6 background were also bred in our animal facility at University of Louisville [179]. Rag2 ${ }^{-/}$mice in C57BL/6 background were purchased from Taconic (Germantown, NY). BLT1 ${ }^{-1-} \mathrm{CXCR}^{-1-}$ double knockout mice were generated by crossing $\mathrm{BLT}^{-/-}$and $\mathrm{CXCR}^{-/-}$mice at our animal facility. All animals were cared for in accordance with institutional and National Institute of Health guidelines and under IACUC protocol. B16 melanoma cell line was purchased from American Type Culture Collection (Manassas, VA) and cultured in complete RPMI media supplemented with 10\% FBS.

\section{Reagents:}

Fluorochrome-conjugated Abs (anti-CD45.2-PE-Cy7, anti-CD3-APC-Cy7, antiCD4-APC, anti-CD8-PerCP-Cy5.5, NK1.1-PE, CD11b-APC, Ly6G-PE, Ly6CFITC, CXCR3-PE, IFNy APC, TNFa APC-Cy7, Streptavidin-APC and 7-AAD) 
were purchased from BD PharMingen and eBioscience. Trp-2 peptide (Trp2180: SVYDFFVWL) was purchased from Peptide 2.0 Inc. Anti-mouse BLT1 antibody conjugated to biotin was developed in the lab (unpublished data). Anti-m-OX-40 agonistic Ab (Clone OX-86) and Anti-m-PD-1 antagonistic Ab (Clone: RMP1-14) were purchased from BioXcell. RT primers for IFN- $\mathrm{y}$, granzyme B, and IL-2 genes were obtained from Real Time Primers, LLC.

\section{Tumor model and vaccinations:}

Naive WT, $\mathrm{BLT}^{-1^{-}}, \mathrm{CXCR}^{-/-}$and $\mathrm{BLT1}^{-1-} \mathrm{CXCR}^{-/-}$mice were challenged with $10^{5}$ live B16 melanoma cells by reconstituting in $200 \mu \mathrm{l}$ PBS and injecting subcutaneously at the right flank of mice to form tumors. For sub-lethal tumor dose experiments, $4 \times 10^{4}$ live B16 cells were injected s.c. Tumor diameter was measured every alternate day using a calliper. Average tumor diameter was calculated by measuring two perpendicular diameters and tumor area was calculated by multiplying the two perpendicular diameters. For survival experiments, mice were allowed to reach $15 \mathrm{~mm}$ tumor diameter as experimental endpoint. Percentage survival was calculated and plotted using Kaplan Meier survival plots. Tumor bearing animals were euthanized once the tumors reached $15 \mathrm{~mm}$ or $7-9 \mathrm{~mm}$ diameter or earlier if they showed any signs of discomfort. For in vivo-cytotoxicity studies, animals were immunized s.c. with $50 \mu \mathrm{g} /$ mice Trp-2 peptide and $100 \mu \mathrm{g} /$ mice OX-86 Ab (Clone OX-86, BioXcell) in PBS or PBS alone as control. For vaccination studies; $\mathrm{WT}, \mathrm{BLT}^{-/}, \mathrm{CXCR}^{-/-}$and $\mathrm{BLT} 1^{--}$ $\mathrm{CXCR}^{-/-}$were challenged with $10^{5}$ live B16 cells s.c. on the right flank on day 0 . On day +5 and day +15 post tumor challenge, the mice were vaccinated i.v. with 
$50 \mu \mathrm{g} /$ mice Trp-2 peptide and $100 \mu \mathrm{g} /$ mice anti-PD-1 antagonistic Ab (clone RMP1-14, BioXcell). The control mice were administered with PBS. The tumor growth was monitored every alternate day. The mice were euthanized when the knockout animals or the control unvaccinated animals reached $15 \mathrm{~mm}$ tumor diameter. Tumors, blood, spleen and tumor draining lymph nodes (TdLN) were then analyzed for $\mathrm{CD}^{+} \mathrm{T}$ cells by flow cytometry.

\section{Flow Cytometry:}

Tumors were harvested and cut into small pieces after removal of connective tissue and tissue stroma. To obtain single cell tumor suspension, the small tumor pieces were incubated in an enzyme mixture consisting of Collagenase A (2 $\mathrm{mg} / \mathrm{ml})$ and DNase-I $(1 \mathrm{mg} / \mathrm{ml})$ in incomplete RPMI medium for $1 \mathrm{hr}$ at $37^{\circ} \mathrm{C}$ on a rocking platform. After $1 \mathrm{hr}$ digestion, single cell suspension was obtained by passing the digested tissue through $40 \mu \mathrm{m}$ nylon mesh and the resultant cells washed twice in PBS before staining for flow cytometry. Cells were stained with fluorochrome labelled anti-mouse Ab like CD45.2, CD3, CD4, CD8, NK1.1, CD11b, Ly6G, Ly6C, etc. Two million total tumor cells were stained and analyzed using multi-parameter flow cytometry. Similarly, spleen and tumor draining lymph nodes (TdLN- inguinal, brachial and axillary) were harvested, processed into single suspension, stained and analyzed via flow cytometry. For intracellular cytokine staining single cell suspensions from tumor, spleen and TdLNs were stimulated with cell stimulation cocktail (eBiosciences, 500X used at $1 \mathrm{X}$ ) consisting of PMA $(40.5 \mu \mathrm{M})$, lonomycin $(670 \mu \mathrm{M})$ and protein transport inhibitors - Brefeldin A (5.3 mM) and Monensin (1 mM) for 6hrs at $37^{\circ} \mathrm{C}, 5 \% \mathrm{CO}_{2}$. After 6 
hrs the cells were harvested and washed, surface stained with CD45, CD3 and CD8 and fixed and permeabilized (IC fixation and Permeabilisation buffer eBiosciences) and stained for IFNy and TNFa using anti-mIFNy Ab and antimTNFa Ab (BD Biosciences). Isotype controls with the same fluorochrome were used as controls. Cells were acquired using FACS Canto II machine and analyzed by FlowJo (TreeStar) software.

\section{Immune-fluorescence microscopy:}

Immune-fluorescence staining for $\mathrm{CD}^{+} \mathrm{T}$ cells in the tumors of $\mathrm{WT}, \mathrm{BLT}^{-/-}$and $\mathrm{CXCR}^{-1-}$ mice was analyzed using Nikon-A1R Confocal microscope. Tumors were embedded in OCT medium and snap frozen in liquid nitrogen and later cut into $5 \mu \mathrm{m}$ sections using a cryostat. Sections were fixed using ice-cold acetone and then blocked using 1X PBS supplemented with 3\% BSA and $5 \%$ goat serum for $1 \mathrm{hr}$ at RT. To stain for $\mathrm{CD} 8^{+} \mathrm{T}$ cells, the sections were incubated with rat antimouse CD8a Ab (BD Pharmingen) in 1X PBS + 3\% BSA for $1 \mathrm{hr}$ at RT. After 3 washes with PBS, the sections were then incubated with the secondary Ab goat anti-rat Alexa $594(2 \mathrm{mg} / \mathrm{ml}$, Invitrogen). After washing with PBS, the sections were mounted with Vectashield mounting medium containing DAPI (Vector Labs) and analyzed at 200X magnification. A minimum of 4 fields for each tumor section was analyzed. 


\section{Real-time PCR}

Total RNA from the excised tumors was isolated using Trizol followed by RNase mini prep kit from Qiagen. The RNA was treated with DNase using Turbo DNAse kit (Ambion). For quantitative real-time PCR, $1 \mu \mathrm{g}$ total RNA was reverse transcribed in $50 \mu \mathrm{l}$ reaction using TaqMan reverse transcription reagents (Applied Biosystems) using random hexamer primers. A total of $2 \mu \mathrm{l}$ cDNA and the $1 \mu \mathrm{M}$ real-time PCR primers were used in a final $20 \mu \mathrm{l} \mathrm{PCR}$ reaction with "power SYBR-green master mix" (Applied Biosystems). The real-time primers were purchased from Real Time Primers, LLC (Elkins Park, PA). The sequence of the primers will be provided upon request. Real-time PCR reaction was performed in Bio-Rad CFX-96 Real Time System. Expression of the target genes was normalized to GAPDH and displayed as fold change relative to the WT sample. Data are representative of tumors isolated from at least five different mice for each genotype.

\section{In vivo cytotoxicity assay:}

A standard in vivo-cytotoxicity assay was performed by injecting peptide pulsed target cells into immunized mice as previously described [176]. WT, BLT1 $1^{-/}$, $\mathrm{CXCR}^{-/-}$and $\mathrm{BLT}^{-/-} \mathrm{CXCR}^{-/-}$recipient mice were immunized s.c. with 50 $\mu \mathrm{g} / \mathrm{mice}$ of Trp-2 peptide and an adjuvant i.e. $100 \mu \mathrm{g} / \mathrm{mice}$ anti-OX-40 agonistic Ab (Clone OX-86, BioXcell). 7 days later, C57BL/6 splenocytes were divided into $\mathrm{CFSE}_{\text {high }}$ and $\mathrm{CFSE}_{\text {low }}$ populations by staining with $2.5 \mu \mathrm{M}$ and $0.25 \mu \mathrm{M}$ CFSE fluorescent dye. CFSE ${ }_{\text {high }}$ cells were pulsed with $2 \mu \mathrm{g} / \mathrm{ml}$ Trp-2 peptide for $90 \mathrm{~min}$ 
at $37^{\circ} \mathrm{C}$ in a $5 \% \mathrm{CO}_{2}$ incubator. CFSE high and low cells were extensively washed and mixed at 1:1 ratio and injected i.v. into the immunized WT, BLT1 ${ }^{-1}$, $\mathrm{CXCR}^{-1-}$ mice. Their spleens were harvested after two days and analyzed by flow cytometry to determine the ratio of $\mathrm{CFSE}_{\text {high }} / \mathrm{CFSE}_{\text {low }}$ target cells and percent killing. The percentage of in vivo killing was calculated by the following formula:

$\left[1-\left(\left[C F S E^{\text {high }} /\right.\right.\right.$ CFSE $^{\text {low }}$ for experimental]/[CFSE ${ }^{\text {high }} /$ CFSE $^{\text {low }}$ for naive $\left.\left.]\right)\right] \times 100$.

\section{Purification of $\mathrm{CD}^{+} \mathrm{T}$ Cells}

CD8+ $T$ cells were isolated from spleen and tumor draining lymph nodes of tumor bearing (3-4mm tumor diameter) $\mathrm{WT}, \mathrm{BLT}^{-{ }^{--}}, \mathrm{CXCR}^{-/-}$or $\mathrm{BLT} 1^{-\mathrm{CXCR}^{-/-}}$ double knockout mice using cell sorting using magnetic beads for CD8 (MACS, Miltenyi Biotec). In co-transfer experiments, tumor bearing UBC-GFP mice were used to obtain $\mathrm{CD}^{+} \mathrm{T}$ cells instead of WT mice. Briefly, spleen or lymph nodes were crushed and passed through $70 \mu \mathrm{m}$ strainer (Corning). The red blood cells were lysed with RBC lysis buffer (Biolegend). After lysis step, cells were resuspended in Automax buffer (Miltenyi Biotec) containing $0.5 \%$ FBS and incubated with $\mathrm{CD}^{+} \mathrm{a}$ (Ly-2) micro beads (Miltenyi Biotec). The $\mathrm{CD}^{+} \mathrm{T}$ cells were isolated as described by the manufacturers protocol and were positively selected on MACS MS columns. The purified $\mathrm{CD}^{+} \mathrm{T}$ cells were $>98 \%$ pure as analyzed by Flow Cytometer. 


\section{Adoptive transfer studies in Rag2 ${ }^{-/-}$mice:}

Rag2 $^{-/}$immune-deficient mice were challenged s.c. with $10^{5}$ live B16 cells. Two days later, $\mathrm{CD}^{+} \mathrm{T}$ cells were isolated from the spleen and TdLN of tumor bearing $\mathrm{WT}, \mathrm{BLT} 1^{-/}, \mathrm{CXCR} 3^{-/}$or $\mathrm{BLT} 1^{-/} \mathrm{CXCR} 3^{-/}$mice by magnetic sorting using CD8a-Ly2 microbeads (Miltenyi Biotec) with $>98 \%$ purity. 1 million purified CD8 ${ }^{+}$ T cells were injected i.v. into the Rag2 ${ }^{-/-}$mice challenged with live B16 tumors and vehicle alone i.e. PBS was used as the control. Tumor growth was monitored every alternate day. Animals were euthanized once they reached $15 \mathrm{~mm}$ tumor diameter and TdLNs as well as tumors were analyzed for $\mathrm{CD}^{+} \mathrm{T}$ cell numbers. For, co-transfer experiments, UBC-GFP mice were used as WT mice in order to distinguish between WT and knockout (non-GFP) $\mathrm{CD}^{+} \mathrm{T}$ cells. Rag2 ${ }^{-/-}$mice were challenged with $10^{5}$ live B16 cells. Two days later, $\mathrm{CD}^{+} \mathrm{T}$ cells were isolated from tumor bearing WT (UBC-GFP), $\mathrm{BLT}_{1}{ }^{-/}, \mathrm{CXCR}^{-/}$and $\mathrm{BLT} 1^{-/}$ $\mathrm{CXCR}^{-/-}$mice. 1 million total $\mathrm{CD} 8^{+} \mathrm{T}$ cells consisting of WT $\left(\mathrm{GFP}^{+}\right)$and either

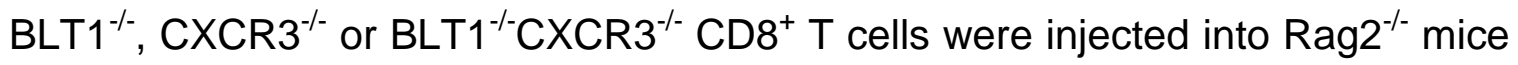
in equal proportion and tumor growth was monitored. Animals were euthanized once they reached $7-9 \mathrm{~mm}$ diameter. Spleen, blood, TdLN and tumors were harvested and $\mathrm{CD}^{+} \mathrm{T}$ cells were analysed for $\mathrm{GFP}^{+}$(WT) and GFP' (knockout) populations. 


\section{Statistical Analysis:}

Statistical analysis was done using the Student $t$ test and Mann Whitney $U$ test. The survival assays were analyzed using long-rank test in Graph Pad Prism software. Student's t-test were used for comparisons between two experimental groups, with a $p$ value of $<0.05$ considered as significant using Graph Pad Prism software $\left({ }^{* * *}=p<0.001 ;{ }^{* *}=p<0.01,{ }^{*}=p<0.05\right)$. Error bars represent $\pm S D$. 


\section{CHAPTER IV}

\section{BLT1 AND CXCR3 REGULATE ANTI-TUMOR IMMUNITY BY FACILITATING CD8 ${ }^{+}$T CELL MIGRATION TO TUMORS}

\section{INTRODUCTION}

Chemokine-chemokine receptor pathways are one of the major factors governing CTL recruitment to tumors and anti-tumor immunity $[40,75]$. Till date only a few chemokine receptor systems are known to regulate $\mathrm{T}$ cell homing to tumors and anti-tumor immunity. Herein, we studied the roles of leukotriene B4 receptor BLT1 and CXCR3 in regulating an endogenous anti-tumor immune response using a syngeneic murine model of B16 melanoma. We hypothesize that BLT1' I- mice would demonstrate defective immune-surveillance and anti-tumor immunity against melanoma tumor, as seen in TC-1 cancer. We also hypothesize that tumors in $\mathrm{CXCR}^{-/-}$mice would rapidly develop compared to WT mice. Experiments involved challenging WT, $\mathrm{BLT}^{-1-}$ and $\mathrm{CXCR}^{-/-}$mice with B16 melanoma cells subcutaneously. Experiments such as assessing tumor infiltrating leukocytes and adoptive transfer of WT or knockout $\mathrm{CD}^{+} \mathrm{T}$ cell in tumor bearing Rag2 ${ }^{-/}$mice were carried out to further investigate the importance of BLT1 and CXCR3 expression on $\mathrm{CD}^{+} \mathrm{T}$ cells for generating effective anti- 
tumor immunity. Adoptive transfer of either $\mathrm{WT}, \mathrm{BLT}^{-/-}$or $\mathrm{CXCR} 3^{-/-}$tumor educated $\mathrm{CD}^{+} \mathrm{T}$ cells in $\mathrm{Rag}^{-/-}$mice somewhat recapitulates the ACT procedure employed in clinic post chemotherapy mediated immune ablation. The effector functions of WT and knockout CTLs in periphery as well as in tumors were then assessed to understand if apart from migration, the effector functions of the $\mathrm{T}$ cells are regulated by BLT1 and CXCR3. This is of relevance, since previous studies have indicated a co-stimulatory role for chemokine receptors on T cells [188]. 


\section{RESULTS}

\section{Defective immune surveillance and anti-tumor immunity in $\mathrm{BLT1}^{-/-}$and $\mathrm{CXCR}^{-/-}$mice:}

Data presented in the previous chapter demonstrated a crucial role for BLT1 in $\mathrm{CD}^{+} \mathrm{T}$ cell migration to tumors and anti-tumor immunity in a viral antigen derived TC-1 cervical cancer model. To determine the requirement for BLT1 and CXCR3 in mediating anti-tumor immunity in an autologous (non-viral) tumor model, syngeneic spontaneous B16 melanoma murine model was employed. $\mathrm{WT}, \mathrm{BLT}^{-/}$and $\mathrm{CXCR}^{-/}$mice were subcutaneously challenged with either a lethal tumor dose $\left(10^{5}\right.$ cells $)$ or sub-lethal tumor dose $\left(4 \times 10^{4}\right.$ cells $)$ of B16 cells. $\mathrm{BLT}^{-/}$and $\mathrm{CXCR}^{-/-}$mice showed significantly enhanced tumor growth as compared to the WT mice at both doses of tumor challenge (Figure 9A and 9B) and significantly reduced survival as compared to the WT mice at the sub-lethal dose (Figure 9C). At the sub-lethal tumor dose both $\mathrm{BLT1}^{-/-}$and $\mathrm{CXCR}^{-/-}$mice demonstrated $100 \%$ mortality by day 28 post tumor challenge, however, $50 \%$ of the WT mice still survived post day 40 with all of them developing relatively slow growing tumors (Figure 9C). These results suggest that both BLT1 and CXCR3 are crucial for immune surveillance and endogenous anti-tumor response. There was no difference between the tumor kinetics of $\mathrm{BLT}^{-/-}$and $\mathrm{CXCR}^{-/-}$mice at both lethal and sub-lethal tumor doses, suggesting that they both are crucial to an equal extent in achieving effective immune surveillance and anti-tumor immunity. 


\section{Reduced homing of $\mathrm{CD}^{+} \mathrm{T}$ cells into tumors of $\mathrm{BLT}^{-/-}$and $\mathrm{CXCR}^{-/-}$mice}

To explore the basis for enhanced tumor growth in the knockout mice, leukocyte sub-populations in tumors, spleen and TdLN of tumor bearing WT, BLT1 ${ }^{-/}$and $\mathrm{CXCR}^{-/}$mice were profiled by flow cytometry. WT, $\mathrm{BLT}^{-/-}$and $\mathrm{CXCR}^{-/-}$mice were challenged with $10^{5} \mathrm{~B} 16$ cells and the tumors were harvested when the knockout tumors reach 7-9 mm (mid-sized) tumor diameter. Single cell suspensions were obtained from the tumor, spleen and TdLN and stained with CD45.2 for all immune cell populations and CD3, CD4 and CD8 for T cells, NK1.1 for NK cells, CD11b, Ly6G and Ly6C for myeloid cell populations. The $\mathrm{BLT}^{-/}$and $\mathrm{CXCR} 3^{-/-}$tumors showed significant reduction in $\mathrm{CD}^{+} \mathrm{T}$ cell numbers as compared to WT tumors (Figure 10A). Moreover, $\mathrm{CXCR}^{-/}$tumors, but not $\mathrm{BLT1}^{-1-}$ tumors, had significant reduction in other effector cell populations like $\mathrm{CD}^{+} \mathrm{T}$ cell and NK cells as compared to the WT tumors. To ensure that reduced CTL numbers are not a function of differential tumor sizes, TIL infiltration, studies were carried out in size-matched tumors. Similar reduction in $\mathrm{CD}^{+} \mathrm{T}$ cell numbers in tumors of knockout mice as compared to WT mice was observed at size matched (end stage) tumors as well (Figure 10B). The significant reduction in $\mathrm{CD}^{+} \mathrm{T}$ cells in the tumors of $\mathrm{BLT}^{-{ }^{--}}$and $\mathrm{CXCR}^{-{ }^{--}}$mice was confirmed by immune-fluorescence staining and confocal microscopy (Figure 10C). Immune cell profiling in the spleen (Figure 11) and TdLN (Figure 12) revealed that knockout mice had similar percentages of $\mathrm{CD}^{+} \mathrm{T}$ cells, $\mathrm{CD} 4^{+} \mathrm{T}$ cells and $\mathrm{NK}$ cells as compared to WT mice. Myeloid cell populations constitute a significant part of the tumor microenvironment. Analysis of $\mathrm{CD}_{11} \mathrm{~b}^{+}$myeloid cells and 
myeloid derived suppressive cells subsets (MDSC) i.e. CD11 $\mathrm{b}^{+} \mathrm{Ly}_{6 \mathrm{G}^{+}}$ (granulocytic-MDSC) and CD11 $\mathrm{b}^{+} \mathrm{Ly}_{6 \mathrm{C}^{+}}$(monocytic - MDSC) in the tumors of $\mathrm{WT}, \mathrm{BLT}^{-/}$and $\mathrm{CXCR}^{-/}$mice showed no significant differences (Figure 13). These results suggest that enhanced tumor growth in $\mathrm{BLT}^{-/}$and $\mathrm{CXCR}^{-/-}$mice may be related to the reduced numbers of cytotoxic cells as compared to tumors of WT mice.

\section{Effector responses in WT, $\mathrm{BLT1}^{-/-}$and $\mathrm{CXCR3}^{-/-}$mice}

To assess the effector responses controlled by BLT1 and CXCR3, transcript expression of various effector molecules and IFNy regulated genes were analyzed in total tumor RNA of $\mathrm{WT}, \mathrm{BLT}^{-/-}$and $\mathrm{CXCR}^{--}$mice by quantitative real time PCR as described in methods. Transcript expression levels of CTL effector molecules like granzyme-B and IFNy were significantly reduced in tumors of $\mathrm{BLT}^{-/-}$and $\mathrm{CXCR}^{-/-}$mice as compared to WT mice as shown by RTPCR (Figure $14 \mathrm{~A}$ and $14 \mathrm{~B}$ ). Expression of interferon gamma inducible genes like CXCL9 and CXCL10 were also determined. CXCL9, ligand for CXCR3 induced by IFNY was significantly reduced in tumors of both $\mathrm{BLT}^{-/-}$and $\mathrm{CXCR}^{-1-}$ mice while CXCL10, another ligand for CXCR3 was significantly reduced in $\mathrm{CXCR}^{-{ }^{-}}$mice (Figure $14 \mathrm{C}$ and 14D). Significantly less IFNy, Granzyme-b and interferon gamma inducible chemokines CXCL9 and CXCL10 in $\mathrm{CXCR}^{-/-}$mice precludes the infiltration of T cells and NK cells that are major producers of IL-2 and IFNy. CXCR3 ${ }^{-/}$tumors showed significantly reduced IL-2 and prevents the feed-forward loop of $T$ cell infiltration $>I F N Y>C X C L 9 / 10>T$ cell infiltration and reduces the overall effector responses in the tumor. Tumors in $\mathrm{CXCR}^{-{ }^{-}}$mice 
show reduced NK cells and CD4+ $\mathrm{T}$ cells that are major These results suggest defective effector responses in tumors of $\mathrm{BLT}^{-{ }^{--}}$and $\mathrm{CXCR} 3^{--}$mice compared to WT mice reflective of reduction in CTL numbers to tumors.

\section{Effector responses of $\mathrm{WT}, \mathrm{BLT1}^{-/-}$and $\mathrm{CXCR}^{-/-} \mathrm{CD}^{+} \mathrm{T}$ cells}

In order to understand if $\mathrm{BLT1}$ and $\mathrm{CXCR} 3$ receptors contribute to effector functions in the CD8 ${ }^{+} \mathrm{T}$ cellS; IFNy and TNF $\alpha$ effector cytokines were analyzed in tumor infiltrated $\mathrm{CD}^{+} \mathrm{T}$ cells as well as $\mathrm{CD} 8^{+} \mathrm{T}$ cells from spleen and TdLN of tumor bearing mice. Invivo cytotoxicity assay was also performed to examine the cytotoxic ability of WT and knockout $\mathrm{CD} 8^{+} \mathrm{T}$ cells in the spleens of immunized WT and knockout mice.

To assess whether the function of $\mathrm{CD}^{+} \mathrm{T}$ cells in $\mathrm{BLT}^{-/-}$and $\mathrm{CXCR} 3^{-/}$is intact or defective, we assessed the in vivo killing activity of $\mathrm{CD} 8^{+} \mathrm{T}$ cells in $\mathrm{WT}, \mathrm{BLT} 1^{-1-}$ and $\mathrm{CXCR}^{-1-}$ mice. Briefly, $\mathrm{WT}, \mathrm{BLT}^{-1-}$ and $\mathrm{CXCR}^{-/-}$recipient mice were immunized s.c. with $50 \mu \mathrm{g} / \mathrm{mice}$ of Trp-2 peptide and an adjuvant i.e. $100 \mu \mathrm{g} / \mathrm{mice}$ anti-OX-40 agonistic Ab (Clone OX-86, BioXcell). 7 days later, C57BL/6 splenocytes were divided into $\mathrm{CFSE}_{\text {high }}$ and $\mathrm{CFSE}_{\text {low }}$ populations by staining with $2.5 \mu \mathrm{M}$ and $0.25 \mu \mathrm{M}$ CFSE fluorescent dye. $\mathrm{CFSE}_{\text {high }}$ cells were pulsed with $2 \mu \mathrm{g} / \mathrm{ml}$ Trp-2 peptide. CFSE high and low cells were extensively washed and mixed at 1:1 ratio and injected i.v. into the immunized and naïve $\mathrm{WT}, \mathrm{BLT} 1^{-/}$and $\mathrm{CXCR}^{-/-}$mice. Their spleens were harvested after two days and analyzed by flow cytometry to determine the ratio of $\mathrm{CFSE}_{\text {high }} / \mathrm{CFSE}_{\text {low }}$ target cells and percent killing. No significant difference was observed in the killing abilities of WT 
as well as $\mathrm{BLT}^{-/-}$and $\mathrm{CXCR}^{-/-} \mathrm{CD} 8^{+} \mathrm{T}$ cells (Figure $15 \mathrm{~A}-\mathrm{D}$ ). These results suggest that there is no intrinsic defect in the cytotoxic function of the knockout $\mathrm{CD}^{+} \mathrm{T}$ cells in the periphery. However, in the tumor microenvironment, the lack of $\mathrm{CXCR} 3$ receptor on the $\mathrm{CD} 8^{+} \mathrm{T}$ cells may render them defective in terms of IFNy production and effector functions.

Given that BLT1 and CXCR3 are essential for homing into tumors, we sought to determine if the effector functions were also controlled by BLT1 and CXCR3. To measure their cytotoxic function within the tumor, levels of IFNy, an effector cytokine was determined in the $\mathrm{CD} 8^{+} \mathrm{T}$ cells by intracellular cytokine staining. The percent IFNy ${ }^{+}$cells of total $\mathrm{CD}^{+} \mathrm{T}$ cells in the tumors of $\mathrm{CXCR}^{-/-}$mice was significantly reduced as compared to WT mice; but remained similar in the tumors of $\mathrm{BLT}^{-/-}$mice (Figure 16A). In contrast, percent IFNy+ cells of total $\mathrm{CD}^{+} \mathrm{T}$ cells in spleens and TdLNs of tumor bearing $\mathrm{WT}, \mathrm{BLT}^{-1-}$ and $\mathrm{CXCR}^{-/-}$ mice were similar. Interestingly, $\mathrm{CXCR}^{-{ }^{-}} \mathrm{CD}^{+} \mathrm{T}$ cells showed intact TNF $\alpha$ production in the tumor as also in spleen and TdLN (Figure 16B).

\section{Adoptively transferred WT but not $\mathrm{BLT1}^{-/-}$or $\mathrm{CXCR}^{-/-} \mathrm{CD}^{+} \mathrm{T}$ cells delayed tumor growth in Rag2 ${ }^{-/-}$mice}

To further examine the importance of BLT1 and CXCR3 expression on CD8 ${ }^{+} \mathrm{T}$ cells for effective infiltration to tumors, adoptive transfer model involving Rag2 ${ }^{-/}$ mice was employed. Rag2 ${ }^{-/-}$mice have intact innate systems and hence allow us to understand the role of BLT1 and CXCR3 specifically on CD8 ${ }^{+} \mathrm{T}$ cells and their 
function in anti-tumor immunity. Rag2 $2^{-/}$mice were challenged with $10^{5}$ B16 cells and 2 days later were adoptively transferred with tumor educated sorted (>98\% pure) WT or BLT1 ${ }^{-1} \mathrm{CD}^{+} \mathrm{T}$ cells and the tumor growth was recorded. PBS transferred tumor bearing Rag2 ${ }^{-/}$mice served as controls. WT CD8 ${ }^{+} \mathrm{T}$ cells significantly reduced the tumor progression in Rag $2^{-/}$animals. However, BLT1 ${ }^{-/}$ $\mathrm{CD}^{+} \mathrm{T}$ cells failed to retard the tumor growth and showed tumor growth kinetics similar to control Rag2 $2^{-/-}$mice without any transferred $\mathrm{CD}^{+} \mathrm{T}$ cells; reinforcing the crucial role of BL1 in anti-tumor immunity (Figure 17A). CTL infiltration studies showed that BLT1 ${ }^{-/}$CTLs were significantly reduced in tumors of Rag2 $2^{-/}$ mice as compared to WT CTLs. Analysis of $\mathrm{CD}^{+} \mathrm{T}$ cells in TdLN revealed no difference in homeostatic proliferation and numbers between transferred WT and $\mathrm{BLT1}^{-/} \mathrm{CD}^{+} \mathrm{T}$ cells (Figure 17B). This suggests a defective tumor homing ability of $\mathrm{BLT} 1^{-\digamma} \mathrm{CTLS}$.

Similar studies were carried out with WT and CXCR3 ${ }^{-/}$CTLs. CXCR3 ${ }^{--}$CTLs also failed to retard tumor growth in Rag2 ${ }^{-/-}$mice suggesting CXCR3 is crucial in anti-tumor immunity (Figure 18A). This defective anti-tumor response was attributed to significantly reduced levels of $\mathrm{CXCR}^{-/-} \mathrm{CTLs}$ in the tumors, their levels remaining similar in the TdLN suggesting no difference between the proliferation of WT and $\mathrm{CXCR}^{-/-}$CTLs (Figure 18B). These studies demonstrate that expression of both BLT1 and CXCR3 on CTLs is necessary for their effective infiltration to tumors and subsequent anti-tumor immunity. 

A
B
$4 \times 10^{4} B 16$

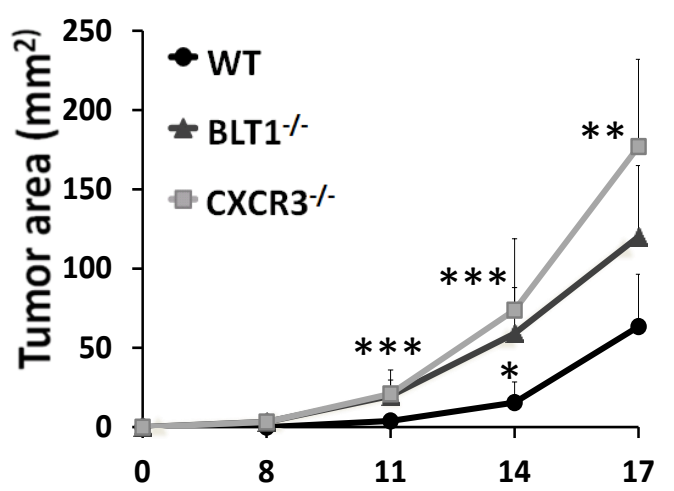

Days post tumor challenge

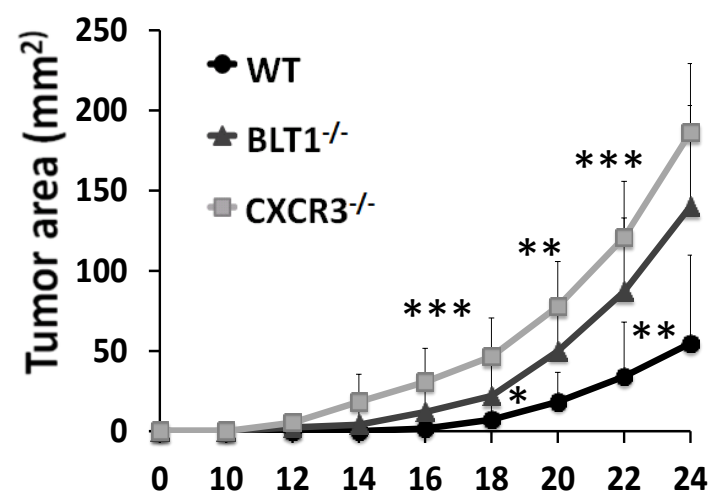

Days post tumor challenge

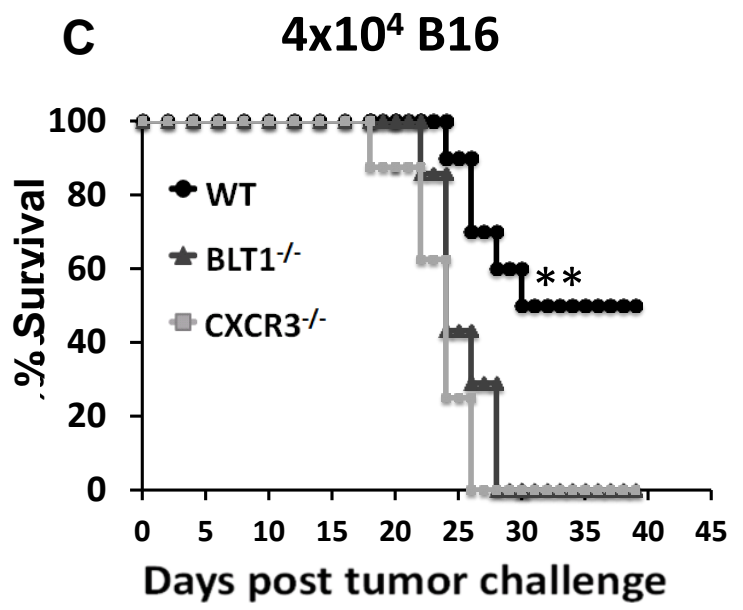

Figure 9: Enhanced tumor growth and reduced survival in $\mathrm{BLT}^{-/-}$and CXCR3 $^{-/-}$mice. A, WT, $\mathrm{BLT1}^{-/}$and $\mathrm{CXCR}^{-/-}$mice were challenged subcutaneously with $10^{5} \mathrm{~B} 16$ cells (lethal dose). Tumor area was determined by multiplication of two perpendicular diameters $(L x W) . n=9$ for each group. B, WT $(n=10), B L T 1^{-/}(n=7)$ and $\mathrm{CXCR}^{-/-}(n=8)$ mice were challenged subcutaneously with $4 \times 10^{4}$ B16 cells (sub-lethal dose) and the tumor area calculated. C, Survival in sub-lethal dose challenge group was monitored till day 45 post tumor 
challenge. Log-rank test and Kaplan-Meier methods were used for survival analyses and student $t$ tests were used for tumor sizes. A, Data is representative of three independent experiments. B, C, Data representative of two independent experiments. 
A

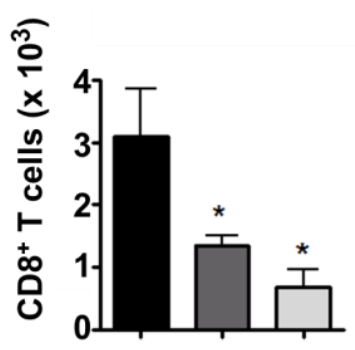

Midsized, day matched tumor
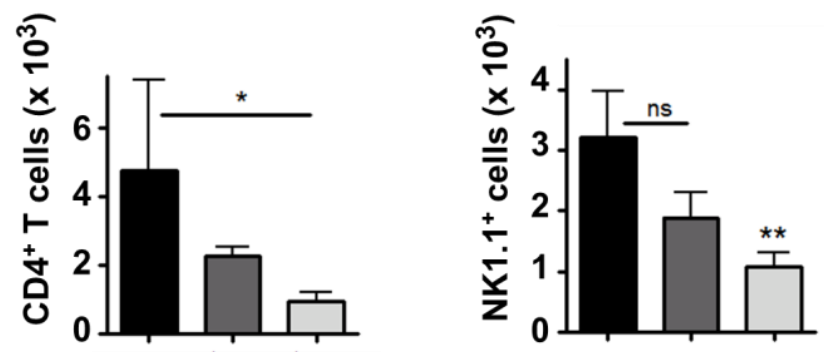

B

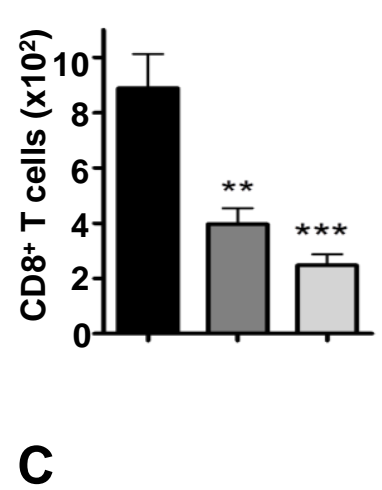

WT

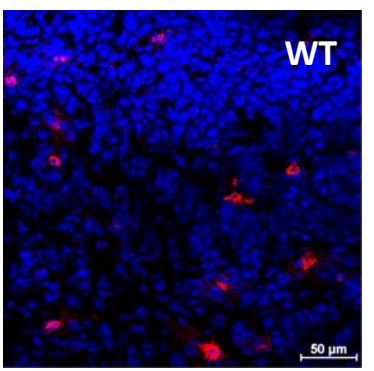

End staged, size matched tumor

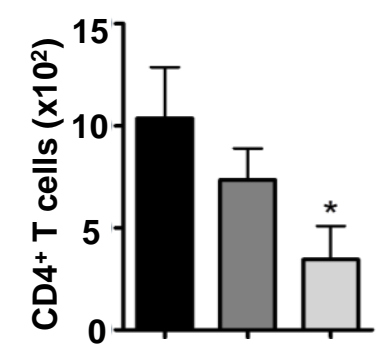

CD8+ $T$ cells - Tumor

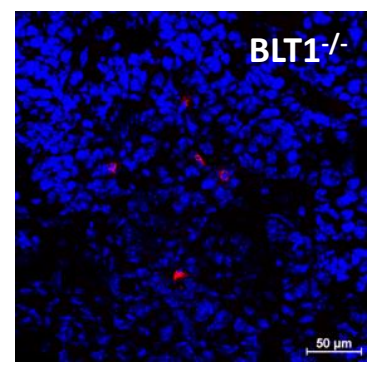

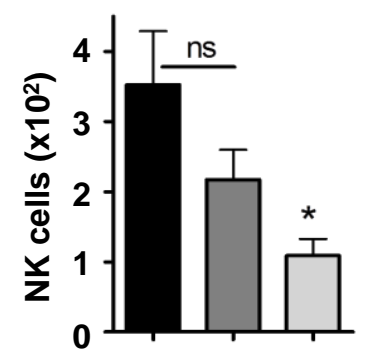

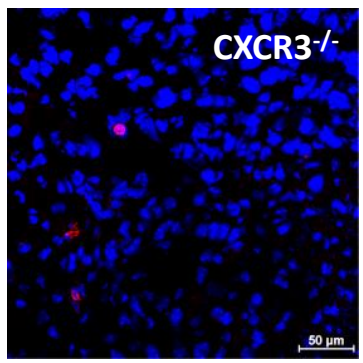

Figure 10: Reduced infiltration of $\mathrm{CD}^{+} \mathrm{T}$ cells in $\mathrm{BLT1}^{-/-}$and $\mathrm{CXCR}{ }^{-/-}$ tumors. A) Reduced CD8 ${ }^{+} \mathrm{T}$ cells in tumors of knockout mice at mid-sized tumor, day matched. Numbers of tumor infiltrating $\mathrm{CD} 8^{+} \mathrm{T}$ cells, $\mathrm{CD} 4^{+} \mathrm{T}$ cells and NK1.1 $1^{+}$cells per million total tumor cells (frequency of total) were analyzed from $\mathrm{WT}, \mathrm{BLT}^{-/}$and $\mathrm{CXCR}^{-{ }^{-}}$mice using standard flow cytometry protocol as 
described in Methods. All the mice were sacrificed and tumors harvested when the knockout tumors reached 7-9mm tumor diameter (mid-sized). B) Reduced $\mathrm{CD}^{+} \mathrm{T}$ cells in size-matched end staged tumors of knockout mice. Numbers of tumor infiltrating $\mathrm{CD} 8^{+} \mathrm{T}$ cells, $\mathrm{CD} 4^{+} \mathrm{T}$ cells and $\mathrm{NK} 1.1^{+}$cells per million total tumor cells (frequency of total) were analyzed from $\mathrm{WT}, \mathrm{BLT} 1^{--}$and $\mathrm{CXCR}^{-/-}$ mice using standard flow cytometry protocol as described in Methods. All tumors were analyzed at large end staged (15 mm tumor diameter), size matched tumors. $n=4$ in each group. C) Representative immunofluorescence staining images of $\mathrm{CD}^{+} \mathrm{T}$ cells in $\mathrm{WT}, \mathrm{BLT1}^{-/-}$and $\mathrm{CXCR}^{-/-}$tumors. Tumors harvested were frozen, sectioned and stained as described in Methods, CD8 represented in Red, DAPI in blue. The images were captured using Nikon A1R confocal microscope. The scale represents $50 \mu \mathrm{M}$. Data representative of three independent experiments. 


\section{Spleen}

A
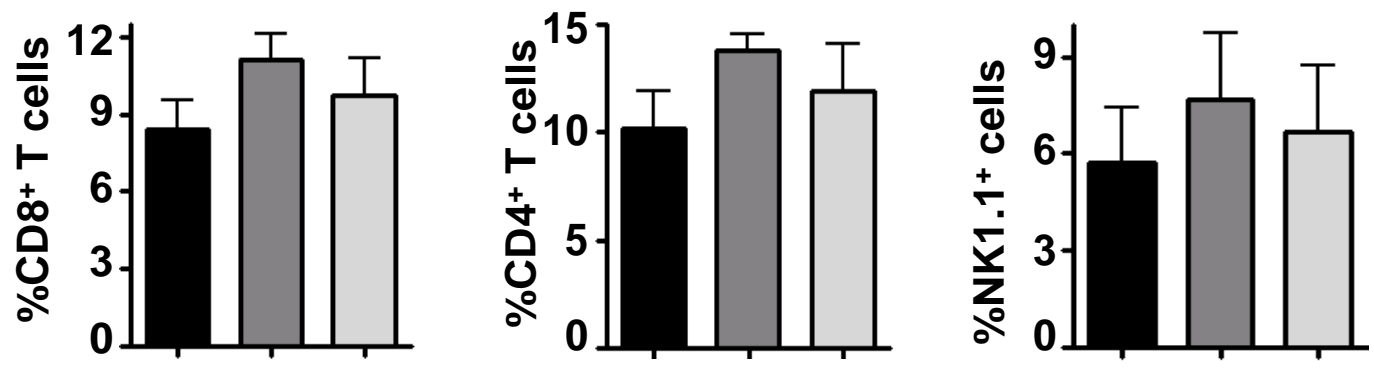

B
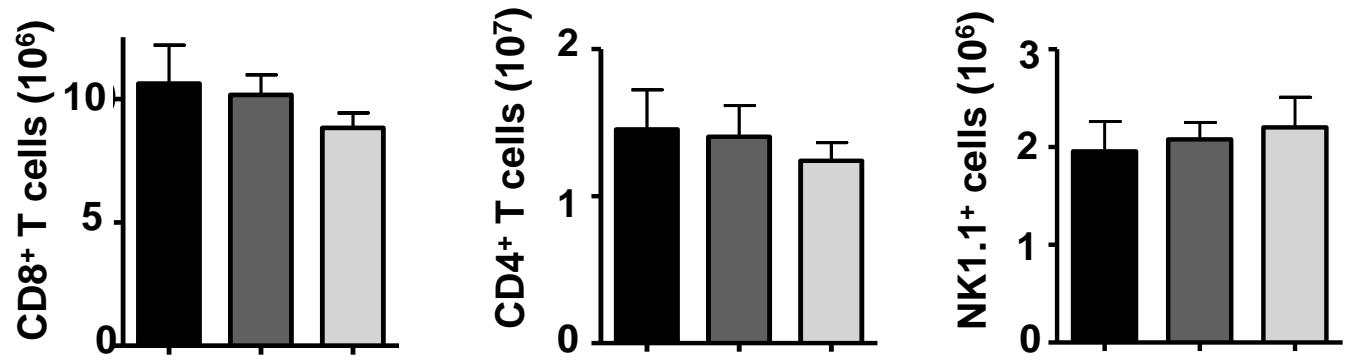

Figure 11: Similar numbers of $\mathrm{CD}^{+} \mathrm{T}$ cells in spleens of WT, BLT1 ${ }^{-{ }_{-}^{-}}$and CXCR3 $^{-/-}$tumor bearing mice: Spleens were harvested, processed into single suspension, stained for CD3, CD8, CD4 and NK1.1 markers and analyzed via flow cytometry as mentioned in the methods. CD8 ${ }^{+} \mathrm{T}$ cells, CD4 ${ }^{+} \mathrm{T}$ cells, NK1.1 ${ }^{+}$ cells in spleen of size-matched, end staged (14-15mm tumor diameter) tumor bearing WT, $\mathrm{BLT1}^{-/}$and $\mathrm{CXCR}^{-/-}$mice. Cells represented as percent of total CD45 ${ }^{+}$cells $(A)$ as well as absolute numbers represented as frequency of total splenocytes (B). $n=4$ in each group. Data is representative of atleast three independent experiments. 
A
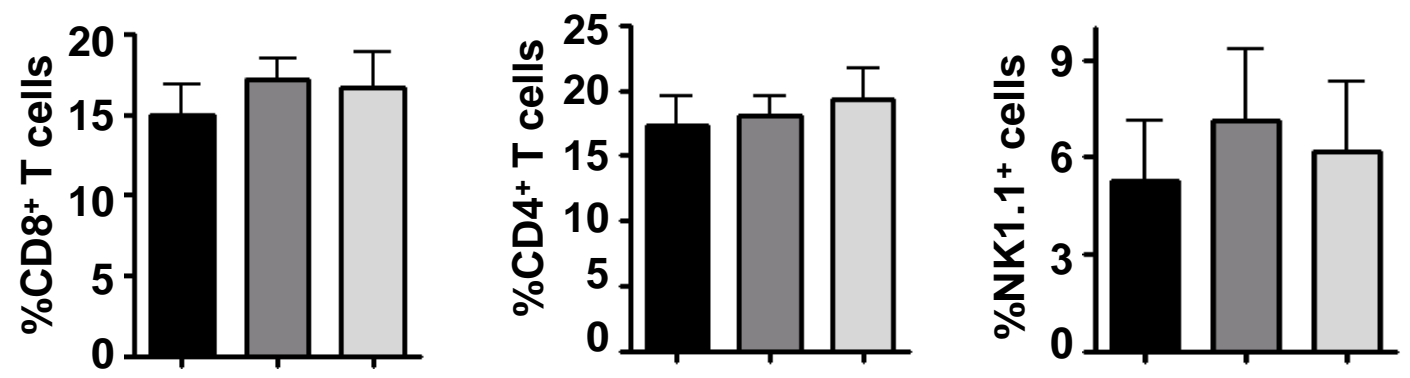

B
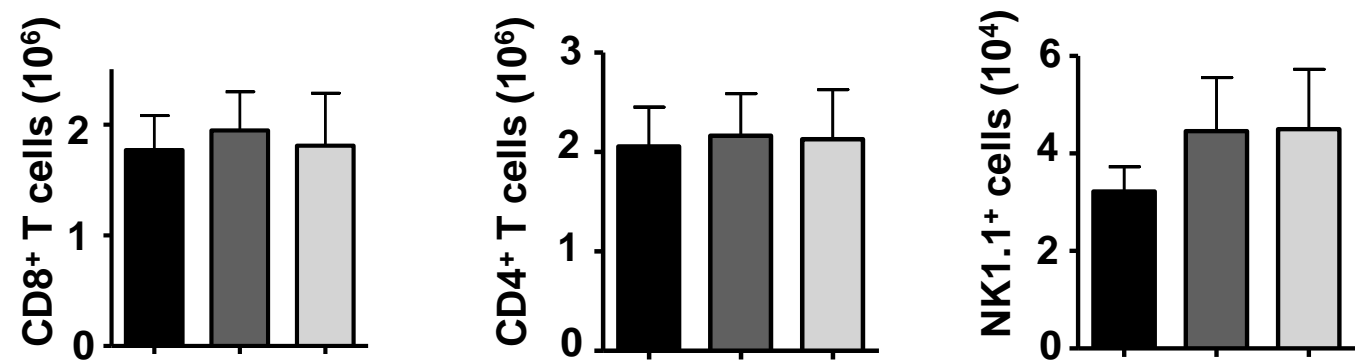

Figure 12: Similar numbers of $\mathrm{CD}^{+} \mathrm{T}$ cells in TdLNs of $\mathrm{WT}, \mathrm{BLT1}^{-/-}$and CXCR3 $^{-/}$tumor bearing mice: Tumor draining lymph nodes (TdLN- inguinal, brachial and axillary) were harvested, processed into single suspension, stained for CD3, CD8, CD4 and NK1.1 markers and analyzed via flow cytometry as mentioned in the methods. CD8 ${ }^{+} \mathrm{T}$ cells, $\mathrm{CD} 4^{+} \mathrm{T}$ cells, NK1.1 $1^{+}$cells in TdLN of size-matched, end staged (14-15mm tumor diameter) tumor bearing WT, BLT1 $1^{-/}$ and $\mathrm{CXCR}^{-/-}$mice. Cells represented as percent of total $\mathrm{CD} 45^{+}$cells $(\mathbf{A})$ as well as absolute numbers represented as frequency of total lymph node cells (B). $\mathrm{n}=4$ in each group. Data is representative of atleast three independent experiments. 


\section{WT}

BLT1\%

CXCR3 $\%$
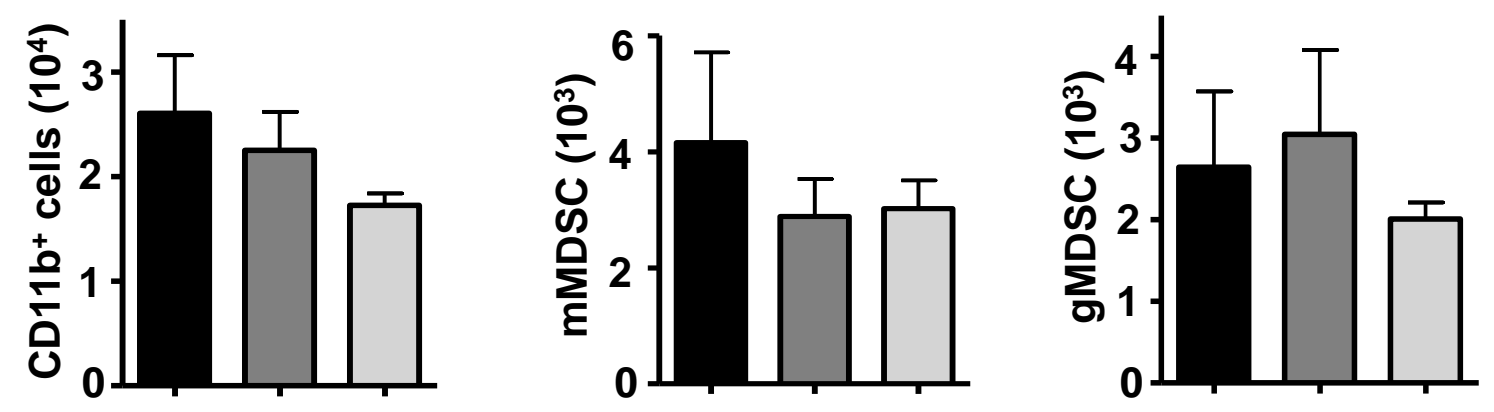

Figure 13: Similar numbers of of myeloid cell subsets in tumors of WT,

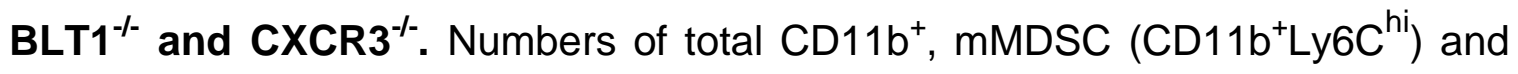
gMDSC (CD11 b+Ly6G $\left.{ }^{h i}\right)$ subsets per million total tumor cells (frequency of total) were analyzed from size matched, end staged (14-15 mm tumor diameter) WT, $\mathrm{BLT}^{-/-}$and $\mathrm{CXCR} 3^{-/-}$mice using standard flow cytometry protocol as described in Methods. $n=4$ in each group. Data is representative of at least three independent experiments. 
A

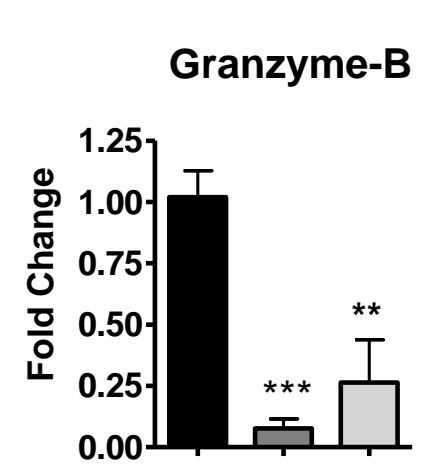

C CXCL9

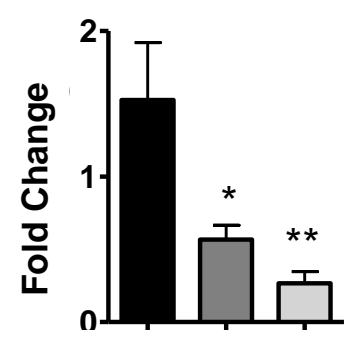

B

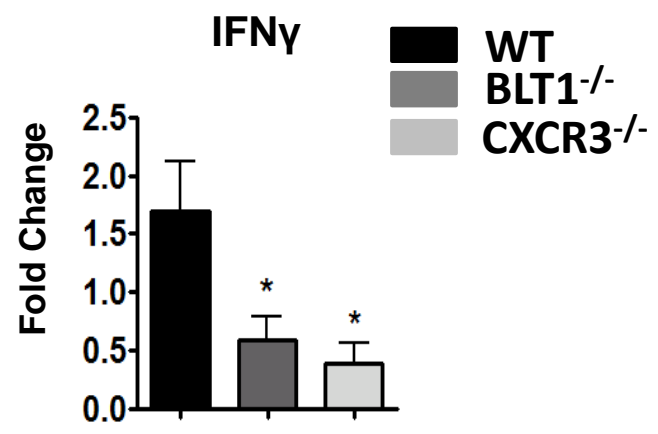

D

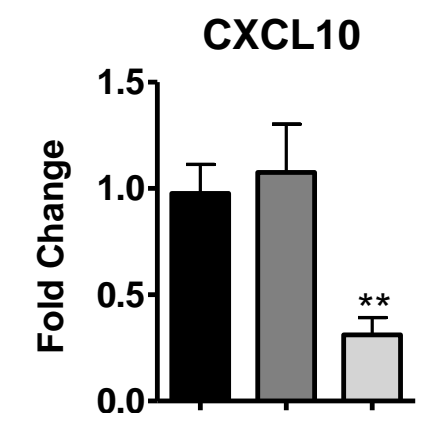

Figure 14: Effector responses in tumors of $\mathrm{WT}, \mathrm{BLT1}^{-/-}$and $\mathrm{CXCR}^{-/-}$mice. Quantitative real time PCR of effector molecules and interferon gamma regulated genes. The levels of Granzyme-B (A), IFNy (B), CXCL9 (C) and CXCL10 (D) mRNA expression in tumors from $\mathrm{WT}, \mathrm{BLT1}^{-/}$and $\mathrm{CXCR}^{-/-}$mice were determined by RT-PCR as described in methods. Expression of the target genes was normalized to GAPDH. Data displayed as fold change relative to the WT sample. $\mathrm{n}=4$ in each group. 
A)

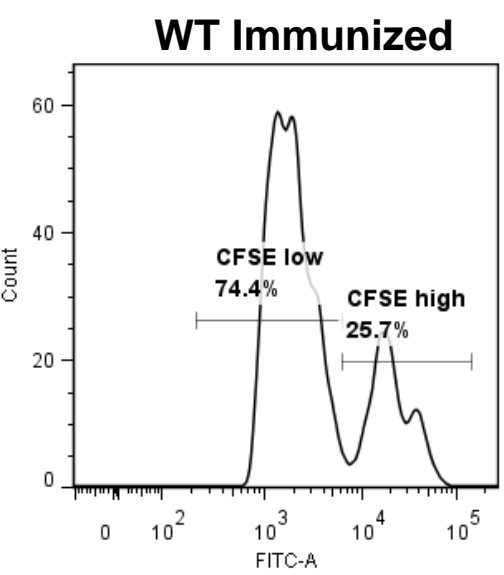

C)

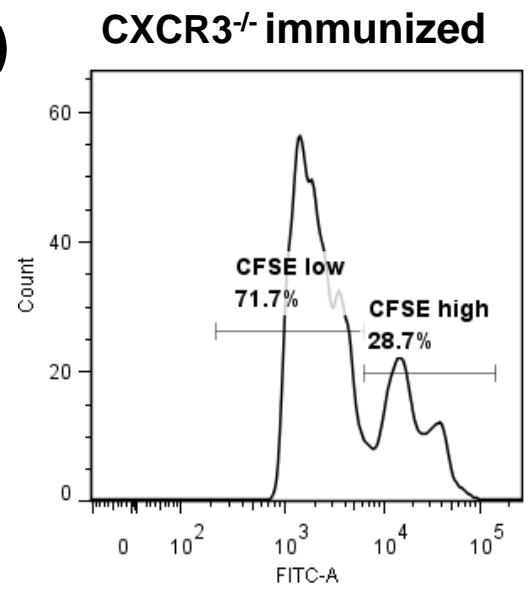

B) BLT1-/immunized

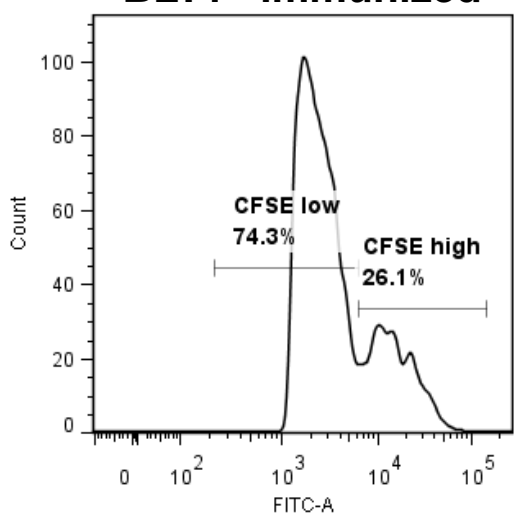

D)

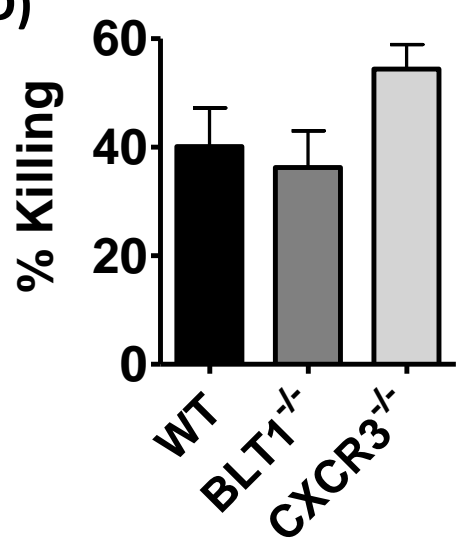

Figure 15: $\mathrm{BLT1}^{-/-}$and $\mathrm{CXCR}^{-/-} \mathrm{CD}^{+} \mathrm{T}$ cells display normal cytotoxic function in the spleen. The $\mathrm{CD}^{+} \mathrm{T}$ cell based In vivo killing assay was performed as described in the Methods. WT, $\mathrm{BLT}^{1-}$ and $\mathrm{CXCR}^{-/-}$recipient mice were immunized s.c. with $50 \mu \mathrm{g} /$ mice of Trp-2 peptide and an adjuvant i.e. 100 $\mu g /$ mice anti-OX-40 agonistic Ab (Clone OX-86, BioXcell). Naïve WT, BLT1 ${ }^{-/}$and $\mathrm{CXCR}^{-1}$ mice were used as controls. Representative histograms of CFSElabeled targets viz. CFSE hi and CFSE low determining the killing in A) Immunized WT mice, B) Immunized $\mathrm{BLT1}^{-/-}$mice and C) Immunized $\mathrm{CXCR}^{-/}$ mice. D) Cumulative levels of percent killing activity by respective CTLs are shown as a bar graph. $\mathrm{n}=4$ in each group. 


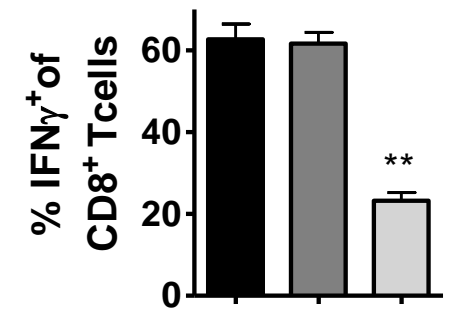

Tumor

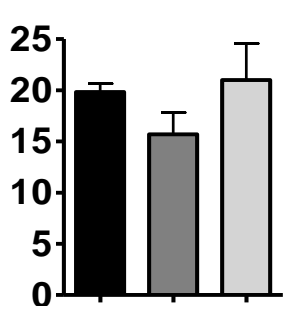

TdLN

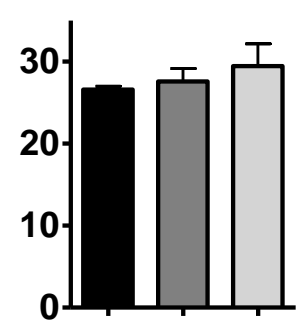

Spleen

B

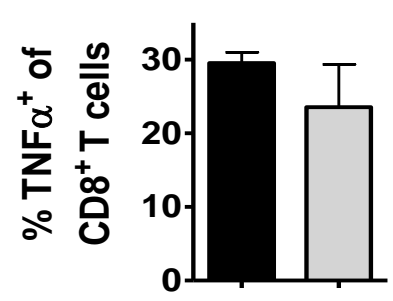

Tumor

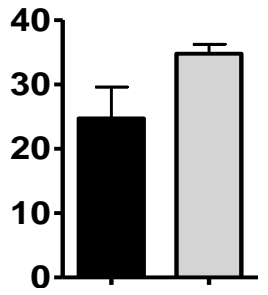

TdLN

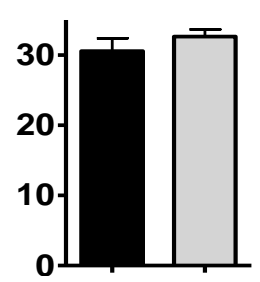

Spleen

Figure 16: Effector functions of $\mathrm{WT}, \mathrm{BLT1}^{-/-}$and $\mathrm{CXCR}^{-{ }^{-}} \mathrm{CD}^{+} \mathrm{T}$ cells in periphery versus tumor. A) Intracellular IFNy staining was performed in WT, $\mathrm{BLT}^{-/-}$and $\mathrm{CXCR}^{-/-}$total tumor cell suspension by ex-vivo stimulation in the presence of PMA and ionomycin as mentioned in the methods. Percent IFNy ${ }^{+}$ cells of total $\mathrm{CD}^{+} \mathrm{T}$ cells were analyzed in tumors (mid-sized tumors). CXCR3 ${ }^{-1-}$ $\mathrm{CD}^{+} \mathrm{T}$ cells in periphery have intact IFNy secretion. Percent IFNy ${ }^{+}$cells of total $\mathrm{CD}^{+} \mathrm{T}$ cells were analyzed in TdLNs and spleens of tumor bearing $\mathrm{WT}$, BLT1 ${ }^{-1-}$ and $\mathrm{CXCR}^{-/}$mice (mid-sized tumor). Data is representative of two independent experiments with $n=4$ animals in each group. B) Intracellular TNF $\alpha$ staining was performed in $\mathrm{WT}, \mathrm{BLT}^{-/-}$and $\mathrm{CXCR}^{-/-}$total tumor cell suspension by ex-vivo stimulation in the presence of PMA and ionomycin as mentioned in the methods. Percent $\mathrm{TNFa}^{+}$cells of total $\mathrm{CD}^{+} \mathrm{T}$ cells were analyzed in tumors, TdLNs and 
spleens of tumor bearing WT and $\mathrm{CXCR}^{-/-}$mice (mid-sized tumor). Data is representative of two independent experiments with $n=4$ animals in each group. 


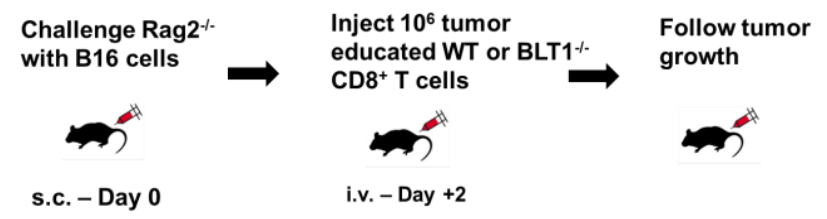

A

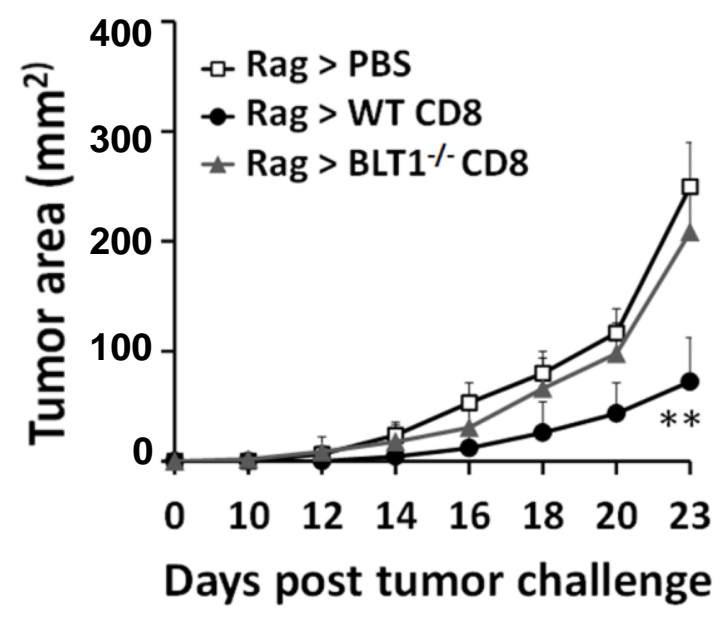

B
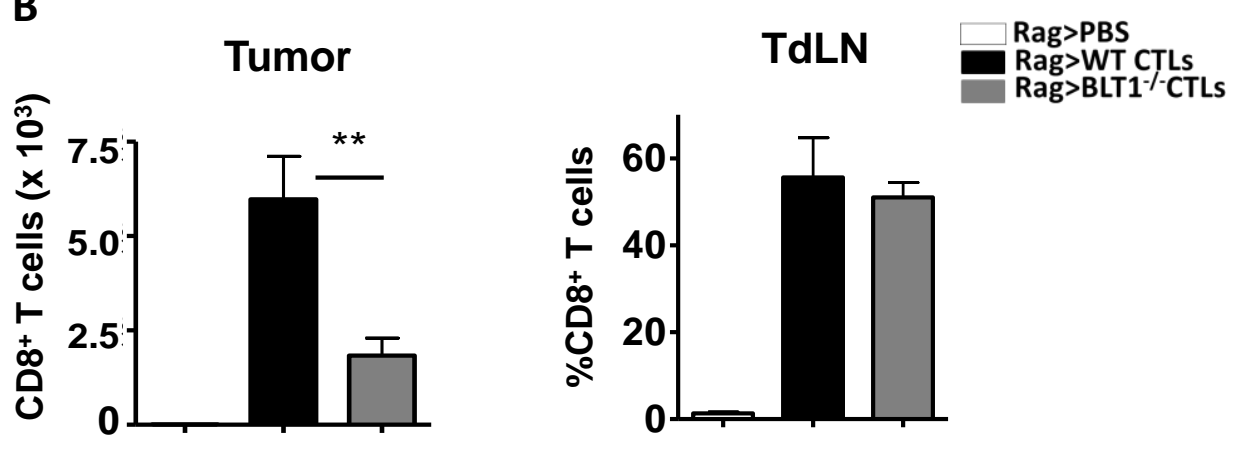

Figure 17: Adoptive transfer of WT but not $\mathrm{BLT1}^{-{ }^{--}}$tumor experienced $\mathrm{CD} 8^{+}$ T cells retarded tumor growth in Rag2 $^{-/-}$mice. Rag2 $2^{-/-}$mice were challenged with $10^{5} \mathrm{~B} 16$ cells. Two days later $\mathrm{CD}^{+} \mathrm{T}$ cells were isolated from the spleen and TdLN of B16 tumor bearing (3-5mm) WT or BLT1 $1^{--}$mice by MACS technique and 1 million isolated $\mathrm{CD}^{+} \mathrm{T}$ cells ( $>98 \%$ purity) or PBS were injected i.v. in tumor inoculated Rag2 ${ }^{-/}$mice. A, Tumor growth kinetics for Rag2 ${ }^{-/-}$mice transferred with either PBS $(n=5)$, WT CD8 ${ }^{+}$T cells $(n=5)$ or $B L T 1^{-1-} C D 8^{+} T$ cells 
$(n=5)$. B, Numbers of $C D 8^{+} T$ cells (frequency of total) per million total tumor cells and percent $\mathrm{CD}^{+} \mathrm{T}$ cells of total $\mathrm{CD}^{+} 5^{+}$cells in $\mathrm{TdLN}$ for WT and $\mathrm{BLT} 1^{-1-}$ transferred $\mathrm{CD}^{+} \mathrm{T}$ cells are shown as cumulative bar graphs. Data is representative of two independent experiments. 


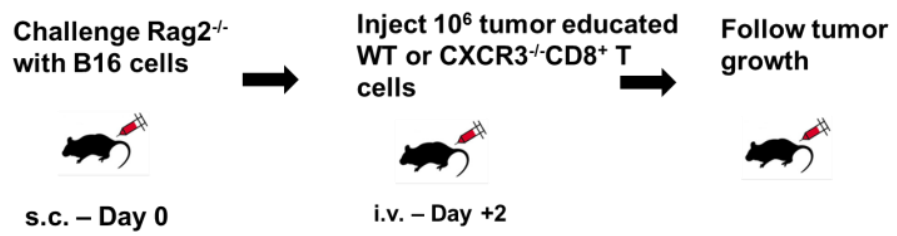

A
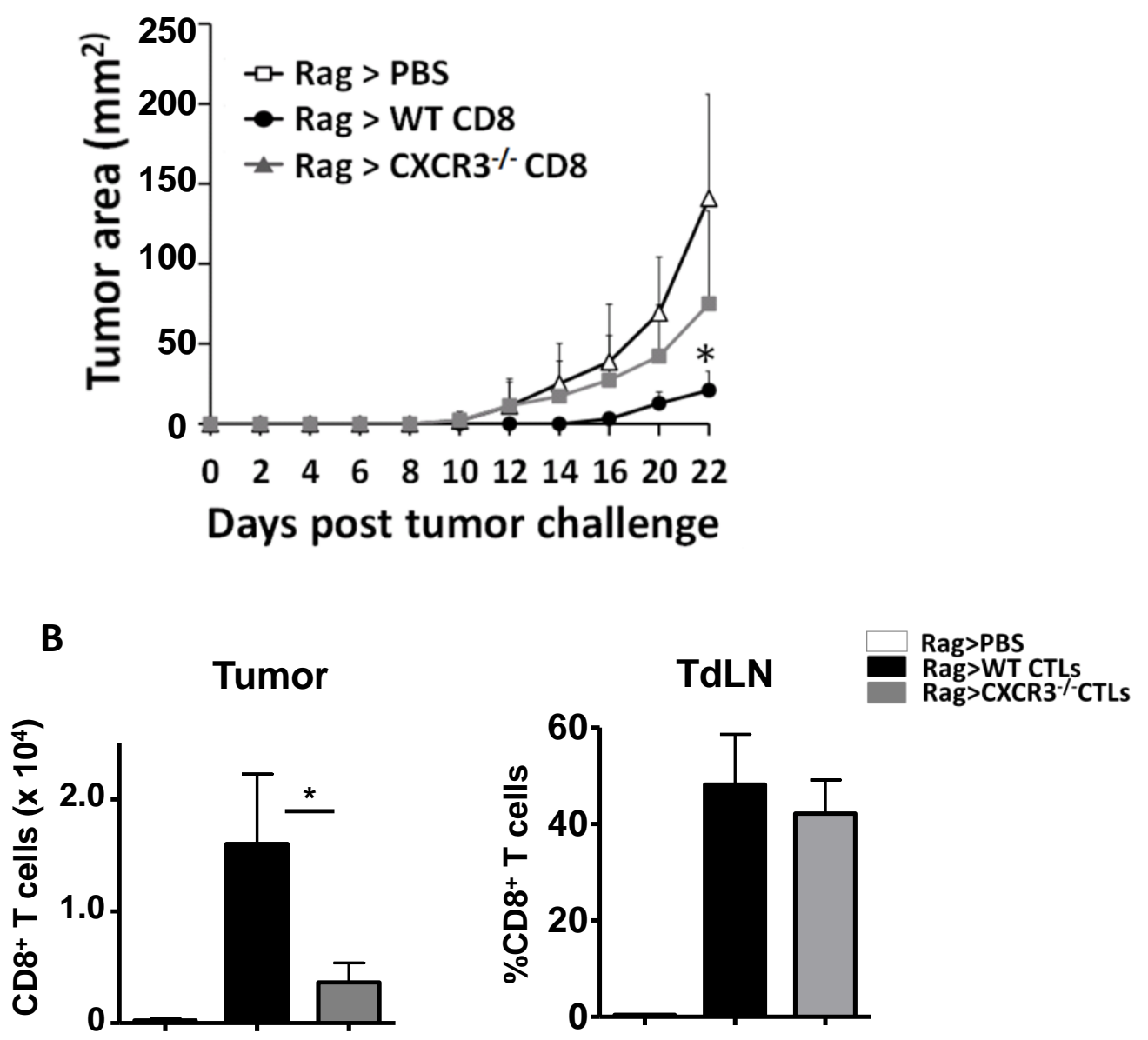

Figure 18: Adoptive transfer of WT but not $\mathrm{CXCR}^{-/-}$tumor experienced CD8 $^{+} \mathbf{T}$ cells retarded tumor growth in Rag2 $^{-/}$mice. Rag2 $2^{-/-}$mice were challenged with $10^{5} \mathrm{~B} 16$ cells. Two days later $\mathrm{CD} 8^{+} \mathrm{T}$ cells were isolated from the spleen and TdLN of B16 tumor bearing (3-5mm) WT or $\mathrm{CXCR}^{-/}$mice by MACS technique and 1 million isolated $\mathrm{CD} 8^{+} \mathrm{T}$ cells ( $>98 \%$ purity) or PBS were injected i.v. in tumor inoculated Rag2 ${ }^{-/-}$mice. A, Tumor growth kinetics for Rag2 ${ }^{-/-}$mice transferred with either PBS $(n=5)$, WT CD8 ${ }^{+} \mathrm{T}$ cells $(n=5)$ or $\mathrm{CXCR}^{-{ }^{-}} \mathrm{CD}^{+} \mathrm{T}$ 
cells $(n=5)$. B, Numbers of CD8 ${ }^{+} T$ cells (frequency of total) per million total tumor cells and percent $\mathrm{CD} 8^{+} \mathrm{T}$ cells of total $\mathrm{CD} 45^{+}$cells in TdLN for WT and CXCR3 ${ }^{-1-}$ transferred $\mathrm{CD}^{+} \mathrm{T}$ cells are shown as cumulative bar graphs. Data is representative of two independent experiments. 


\section{CHAPTER V}

\section{LACK OF BLT1 AND CXCR3 MEDIATED SYNERGISM TO FACILITATE CTL MIGRATION TO TUMORS AND ANTI-TUMOR IMMUNITY}

\section{INTRODUCTION}

The data presented in the previous chapter indicated the individual importance of BLT1 and CXCR3 in regulating CTL migration to tumors and thus anti-tumor immunity. The data indicated that both BLT1 and CXCR3 deficient mice had similar significantly enhanced tumor growth kinetics, compared to WT mice. In this chapter we sought to investigate whether BLT1 and CXCR3 receptor mediated $\mathrm{T}$ cell homing acts in concert or is synergistic in function. For this purpose, $\mathrm{BLT}^{-1-} \mathrm{CXCR}^{-/-}$double deficient mouse was generated by crossing $\mathrm{BLT}^{-/-}$mouse to $\mathrm{CXCR}^{-/-}$mouse.

Tumor growth in double knockout mice was compared to either of the single knockout to infer whether synergism exist with respect to regulation of anti-tumor immunity as well as $\mathrm{CD}^{+} \mathrm{T}$ cell migration to tumors. We hypothesize that BLT1 and CXCR3 mediated regulation of $\mathrm{CD}^{+} \mathrm{T}$ cell migration to tumors and anti-tumor immunity acts in concert. We expect to see a further enhancement of tumor growth in the absence of both BLT1 and CXCR3 receptors. 
Also, the interdependence of BLT1 and CXCR3 mediated regulation of CTL migration to tumors was examined by employing co-transfer strategies of WT $\mathrm{CD}^{+} \mathrm{T}$ cells with either of the knockout cells $\left(\mathrm{BLT1}^{-/-}, \mathrm{CXCR}^{-/-}\right.$or $\mathrm{BLT} 1^{-/}$ $\mathrm{CXCR}^{-/-} \mathrm{CD}^{+} \mathrm{T}$ cells) in equal proportion in the adoptive transfer model employed in previous experiments. WT CTLs facilitating either of $\mathrm{BLT1}^{-1-}$ or $\mathrm{CXCR}^{-/-} \mathrm{CD}^{+} \mathrm{T}$ cell infiltration but not $\mathrm{BLT}^{-{ }^{-} \mathrm{CXCR}}{ }^{--} \mathrm{CD} 8^{+} \mathrm{T}$ cells to tumors would mean a possible interdependence between BLT1 and CXCR3 pathways. We hypothesize that WT $\mathrm{CD}^{+} \mathrm{T}$ cells could facilitate additional BLT1 deficient $\mathrm{CD}^{+} \mathbf{T}$ cells via $\mathbf{C X C R 3}$ receptor. This hypothesis stems from previously published study where BLT1 mediated infiltration of WT neutrophils in $\mathrm{BLT}^{-/}$mice facilitated infiltration of endogenous $\mathrm{BLT}^{-/}$neutrophils to the inflamed joint suggesting that BLT1 expression on neutrophils is essential only for the initial recruitment and other chemokines could then perpetuate the disease progression [180]. We expect that the initial infiltration of WT CTLs to tumors would enable IFN $\gamma>$ CXCL9/10 > CXCR3 mediated T cell homing loop, to enhance the infiltration of additional $\mathrm{BLT1}{ }^{-/} \mathrm{CTLs}$ via $\mathrm{CXCR} 3$ receptor.

These experiments would provide us the plausible combinatorial regulatory mechanisms for BLT1 and CXCR3 mediated T cell homing to tumors as well as anti-tumor immunity. 


\section{RESULTS}

\section{Generation of $\mathrm{BLT1}^{-/-} \mathrm{CXCR}^{-/-}$mice}

BLT1 receptor gene, Ltb4R, is localized to chromosome 14 in mice as well as in humans (autosomal) [179]. On the other hand, CXCR3 gene Cxcr3 is located on the sex-chromosome and is an X-linked gene both in mice and humans [133]. Hence, males are hemizygous for CXCR3 gene and females are homozygous. $\mathrm{BLT1}^{-/}$female mice were bred with $\mathrm{CXCR}^{-/}$male mice to generate $\mathrm{BLT1}$ CXCR3 heterozygous female mice. BLT1-CXCR3 heterozygous females were then bred with $\mathrm{BLT}_{1}{ }^{--}$males to generate $\mathrm{BLT}_{1}{ }^{-/} \mathrm{CXCR} 3^{+/-}$females and $\mathrm{BLT} 1^{-/}$ $\mathrm{CXCR}^{-/-}$males. The $\mathrm{BLT}^{-{ }^{-}} \mathrm{CXCR}^{+/-}$females were then bred with $\mathrm{BLT}^{-/-}$ $\mathrm{CXCR}^{--}$males to generate $\mathrm{BLT} 11^{--} \mathrm{CXCR} 3^{--}$female mice. The $\mathrm{BLT} 1^{--} \mathrm{CXCR}^{-{ }^{-}}$ male and female mice were then bred with each other for several rounds of breeding to generate $\mathrm{BLT}^{-/} \mathrm{CXCR}^{-/}$male and female breeders. The representative breeding scheme is shown in Table 1. The $\mathrm{BLT}^{-1-} \mathrm{CXCR}^{-/-}$mice were born at the expected Mendelian ratios and displayed normal developmental and morphological features.

\section{Accelerated tumor growth and reduced survival in $\mathrm{BLT}^{-/-} \mathrm{CXCR}^{-/-}$mice}

To examine the interdependence of $\mathrm{BLT1}$ and $\mathrm{CXCR} 3$ receptor mediated regulation in anti-tumor immunity, $\mathrm{BLT}^{-{ }^{-} \mathrm{CXCR}^{-/-}}$double knockout mouse (DKO) was generated as discussed. At the sub-lethal dose of B16 cells $\left(4 \times 10^{4}\right)$, $\mathrm{BLT}^{-1-} \mathrm{CXCR}^{-/-}$mice showed significantly enhanced tumor growth as well as 
significantly reduced survival as compared to WT mice (Figure 19 A and B). There was $100 \%$ mortality of $\mathrm{BLT}^{-{ }^{--} \mathrm{CXCR}} 3^{-/-}$mice by day 22 , while $50 \%$ of WT mice still survived at day 35-post tumor challenge (Figure 19B).

To compare the tumor growth kinetics in double knockout mice versus either of the single knockout mice; $\mathrm{WT}, \mathrm{BLT} 1^{-/}, \mathrm{CXCR}^{-/}$and $\mathrm{BLT}^{-/-} \mathrm{CXCR}^{-/-}$mice were challenged with $10^{5}$ B16 cells followed by tumor growth kinetic analysis. The

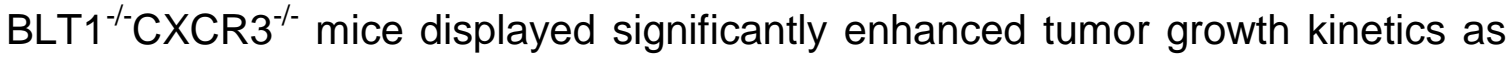
compared to WT mice but similar growth kinetics as compared to $\mathrm{BLT}^{-/}$or $\mathrm{CXCR}^{-/}$mice at lethal tumor dose (Figure 20A). No significant difference was observed in the survival (Figure 20B) or the tumor growth (data not shown) between either of the single knockout mice and double knockout mice even at the sub-lethal dose. Therefore, these results suggest that BLT1 and CXCR3 mediated regulation of anti-tumor immunity may be co-dependent but not additive or synergistic.

\section{WT CTLs do not facilitate knockout CTL infiltration to tumors}

The migration patterns of the WT and knockout $C D 8^{+} \mathrm{T}$ cells co-transferred into Rag2 $^{-/}$mice were determined to explore whether WT CTLs can facilitate the trafficking of knockout CTLs into tumors. Equal proportions of tumor experienced WT CD8 ${ }^{+} \mathrm{T}_{\text {cells }}$ and $\mathrm{BLT1}^{-/}, \mathrm{CXCR}^{-/-}$or $\mathrm{BLT}^{-1-} \mathrm{CXCR}^{-{ }^{--}}$(DKO) $\mathrm{CD}^{+} \mathrm{T}$ cells were introduced intravenously in tumor bearing Rag2 $2^{-/}$mice. UBC-GFP mice were used as WT mice to differentiate between WT (bright green fluorescence) and the knockout $\mathrm{CD}^{+} \mathrm{T}$ cells (Figure 21). $\mathrm{CD}^{+} \mathrm{T}$ cells were stained in tumor, 
blood, spleen and TdLN once the tumor reached 7-9 $\mathrm{mm}$ diameter and the ratio of WT to knockout CD8 ${ }^{+}$T cells was assessed. The ratios of WT to knockout CTLs in TdLN were similar across all the groups and close to 1-1.5 (Figure 22). WT CD8 ${ }^{+}$T cells infiltrated into tumors around 3 fold more as compared to BLT1 ${ }^{-}$ 1- $\mathrm{CD}^{+} \mathrm{T}$ cells, around 8 fold more as compared to $\mathrm{CXCR}^{-/-} \mathrm{CD}^{+} \mathrm{T}$ cells and around 5.5 fold more as compared to $\mathrm{BLT}^{-1}{ }^{-\mathrm{CXCR}} 3^{--} \mathrm{CD}^{+} \mathrm{T}$ cells (Figure 23). In contrast, the numbers of $\mathrm{BLT} 1^{--}, \mathrm{CXCR}^{-{ }^{--}}$, and $\mathrm{BLT}^{-{ }^{--}} \mathrm{CXCR}^{-{ }^{--}} \mathrm{CD} 8^{+} \mathrm{T}$ cells were 1.5 to 3 fold higher than the WT cells in the blood and spleen (Figure 24). These studies suggest that WT cells do not facilitate additional knockout CTL infiltration to tumors. 


\section{BREEDING SCHEME}

\begin{tabular}{|c|c|c|c|}
\hline Cross & Parent (Male) & Parent (Female) & Selected Progeny \\
\hline 1. & CXCR3-/- & BLT1 & BLT1 $^{+/-}$CXCR3 $^{+/-}$Female \\
\hline 2. & BLT1 & $\mathrm{BLT1}^{+/-} \mathrm{CXCR}^{+/-}$ & $\begin{array}{l}\text { a) } \text { BLT1 }^{-/-C X C R 3^{-/-} \text {Male }} \\
\text { b) BLT1 }{ }^{-/-} \mathrm{CXCR}^{+/-} \text {Female }\end{array}$ \\
\hline 3. & BLT1-I-CXCR3--- & BLT1/- CXCR3 ${ }^{+/-}$ & 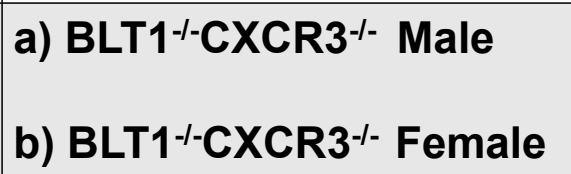 \\
\hline 4. & BLT1 ${ }^{-I-C X C R 3-/-}$ & BLT1 ${ }^{-I-C X C R 3^{-/-}}$ & $\begin{array}{l}\text { BLT1-I-CXCR3 }{ }^{-/-} \text {male and } \\
\text { female pups. }\end{array}$ \\
\hline
\end{tabular}

Table 1: Breeding scheme for generation of $\mathrm{BLT1}^{-/-} \mathrm{CXCR}^{-/-}$mice: Breeding scheme to generate $\mathrm{BLT1} 1^{-1-} \mathrm{CXCR} 3^{-/}$double deficient mice. $\mathrm{BLT1} 1^{-/}$females were bred with $\mathrm{CXCR}^{-/}$males to generate BLT1-CXCR3 heterozygous females. BLT1-CXCR3 heterozygous females were then bred with $\mathrm{BLT1}{ }^{-/}$males to

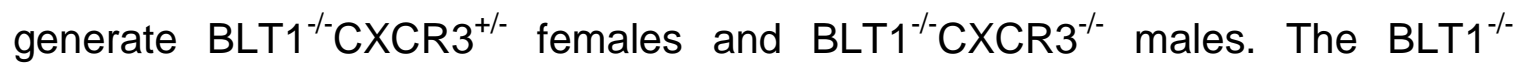
$\mathrm{CXCR}^{+/-}$females were then bred with $\mathrm{BLT}^{-{ }^{--} \mathrm{CXCR}} 3^{-/-}$males to generate $\mathrm{BLT} 1^{-/-}$ $\mathrm{CXCR}^{-/-}$females. The $\mathrm{BLT}^{-1-}{ }^{-\mathrm{CXCR}} 3^{-/-}$males and females were then bred with each other for several rounds of breeding to generate $\mathrm{BLT}^{-/-} \mathrm{CXCR}^{-/-}$male and female breeders. The representative breeding scheme is shown. 
A

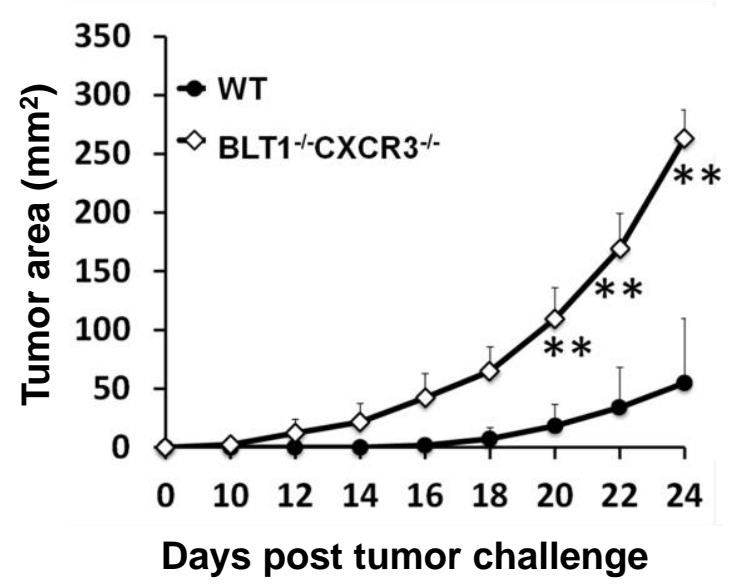

B

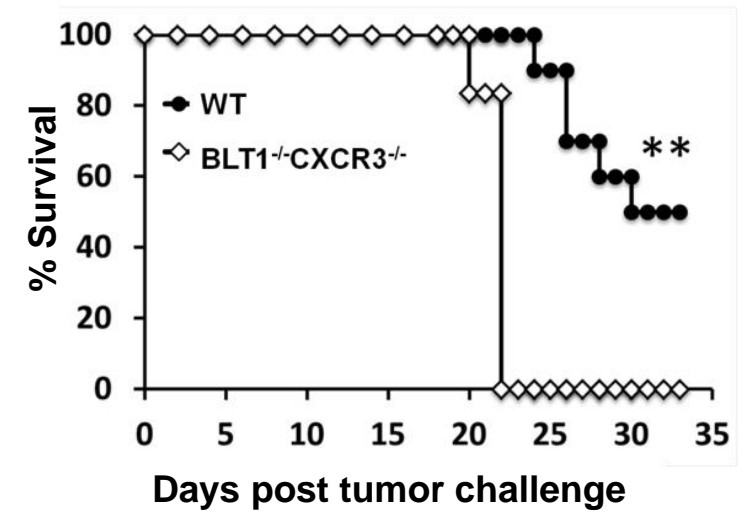

Figure 19: Defective immune surveillance and anti-tumor immunity in BLT1' ${ }^{1-C_{C X R 3}}{ }^{-/}$double knockout (DKO) mice. A, WT $(\mathrm{n}=10)$ and $\mathrm{BLT} 1^{-/} \mathrm{CXCR} 3^{-/-}$ $(n=6)$ mice were challenged subcutaneously with $4 \times 10^{4}$ B16 cells (sub-lethal dose) and the tumor area calculated. Tumor area was measured by multiplication of two perpendicular diameters (LxW). B, Survival in mice with sub-lethal dose of tumor challenge was monitored till day 35 post tumor challenge. Log-rank test and Kaplan-Meier methods were used for survival analyses and student $t$ tests 
were used for tumor sizes. Data is representative of two independent experiments. 
A

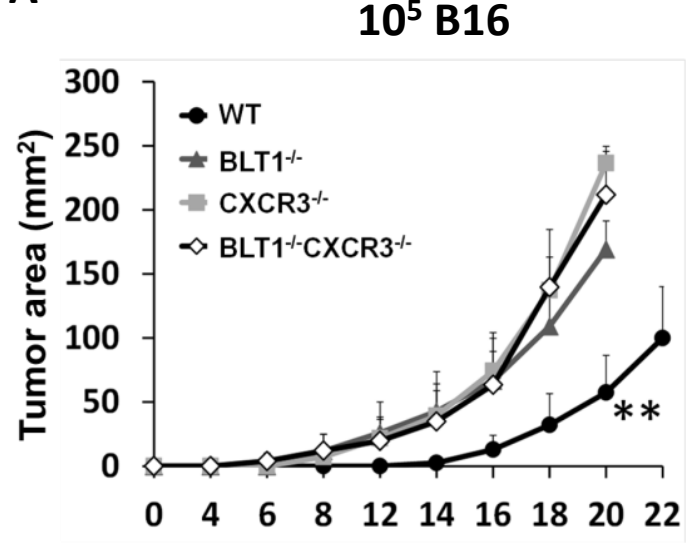

Days post tumor challenge
B

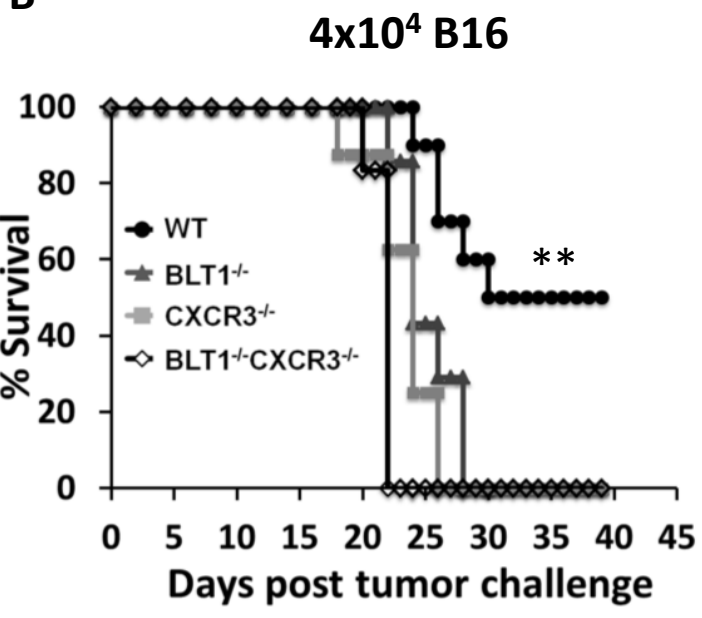

Figure 20: Lack of synergism in BLT1 and CXCR3 mediated regulation in anti-

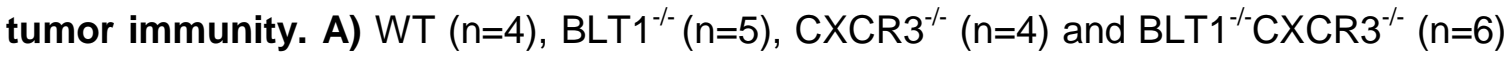
mice were challenged subcutaneously with $10^{5} \mathrm{~B} 16$ cells (lethal dose). Tumor area was measured by multiplication of two perpendicular diameters (LxW). B) WT ( $n=10), B L T 1^{-}$ /- $(n=7), \mathrm{CXCR}^{-/-}(\mathrm{n}=7)$ and $\mathrm{BLT}^{-/} \mathrm{CXCR}^{-/} \quad(n=6)$ mice were challenged subcutaneously with $4 \times 10^{4} \mathrm{~B} 16$ cells (sub-lethal dose). Survival in mice with sublethal dose of tumor challenge was monitored till day 40 post tumor challenge Data is representative of two independent experiments. Log-rank test and KaplanMeier methods were used for survival analyses and student $t$ tests were used for tumor sizes. 
Co-transfer experiments:
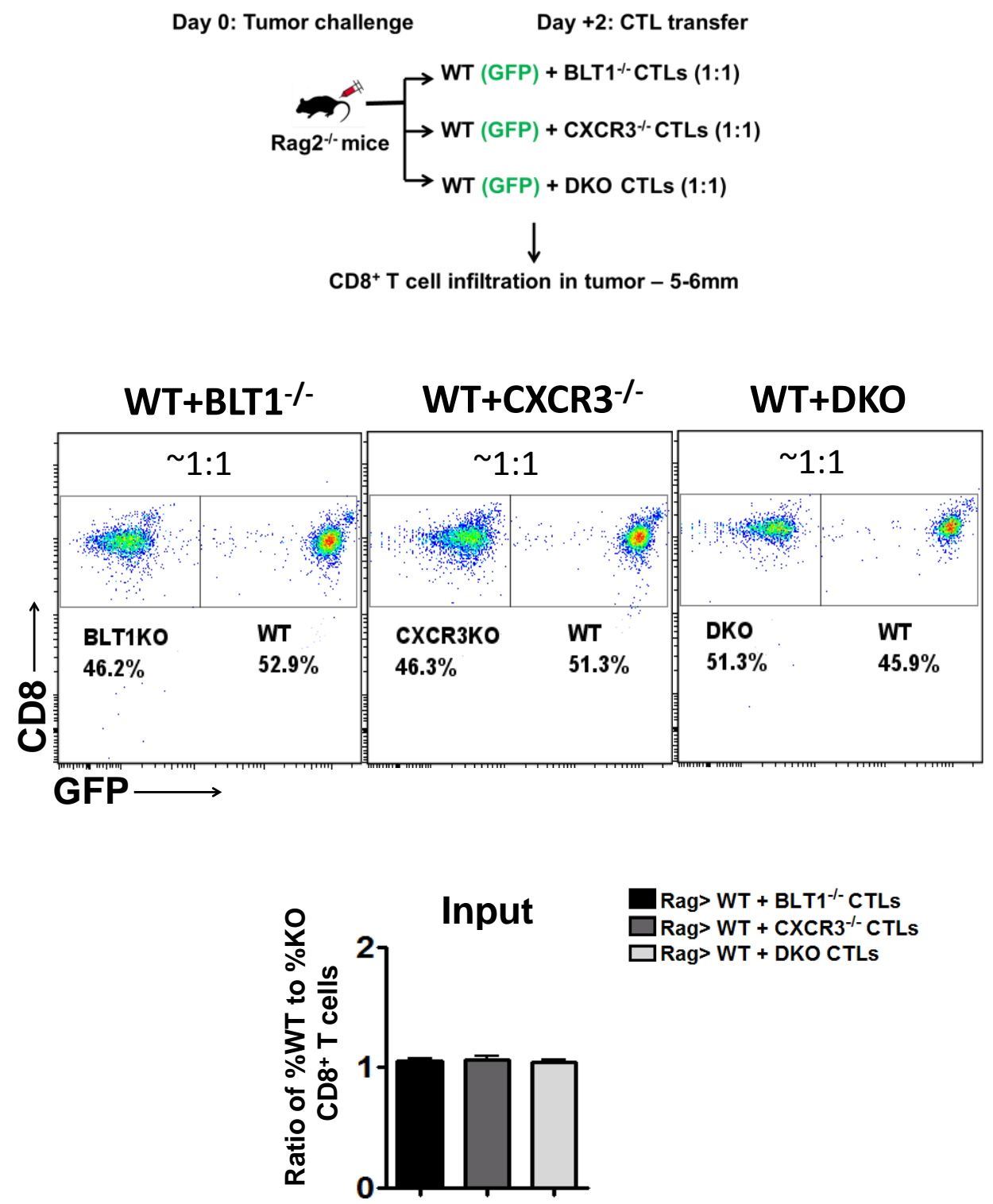

Figure 21: Equal proportions of WT (GFP) and knockout $\mathrm{CD}^{+} \mathrm{T}$ cells adoptively transferred in tumor bearing Rag2 $2^{-/-}$mice. Co-transfer experiments were performed as mentioned in methods. Briefly, $\mathrm{CD} 8^{+} \mathrm{T}$ cells were isolated from tumor bearing $\left(3-5 \mathrm{~mm}\right.$ ) WT (GFP), BLT1 ${ }^{-/-}$(non-GFP), CXCR3 ${ }^{-/}$(non-GFP) mice and $\mathrm{BLT}^{-{ }^{-}} \mathrm{CXCR3}^{-{ }^{-}}$(non-GFP) mice. WT and $\mathrm{KO} \mathrm{CD}^{+} \mathrm{T}$ cells were equally mixed and 1 million cells were injected i.v. in tumor bearing Rag $2^{-/-}$mice. 
Representative dot plots of $\mathrm{GFP}^{+}(\mathrm{WT})$ and $\mathrm{GFP}^{-}(\mathrm{KO})$ CTLs transferred in tumor inoculated Rag2 $2^{-/}$mice and corresponding cumulative bar graph of ratio of \%WT to $\% \mathrm{KO} \mathrm{CD8} 8^{+} \mathrm{T}$ cells (gated on $\mathrm{CD}^{+} \mathrm{CD}^{+}$cells) injected which is equal to 1 . Bar in black represents $\mathrm{WT}+\mathrm{BLT} 1^{-1-} \mathrm{CTL}$ mix; grey bars represent $\mathrm{WT}+\mathrm{CXCR} 3^{-/-}$ CTL mix and light grey bar represents WT + DKO CTL mix. Data is representative of two independent experiments. 

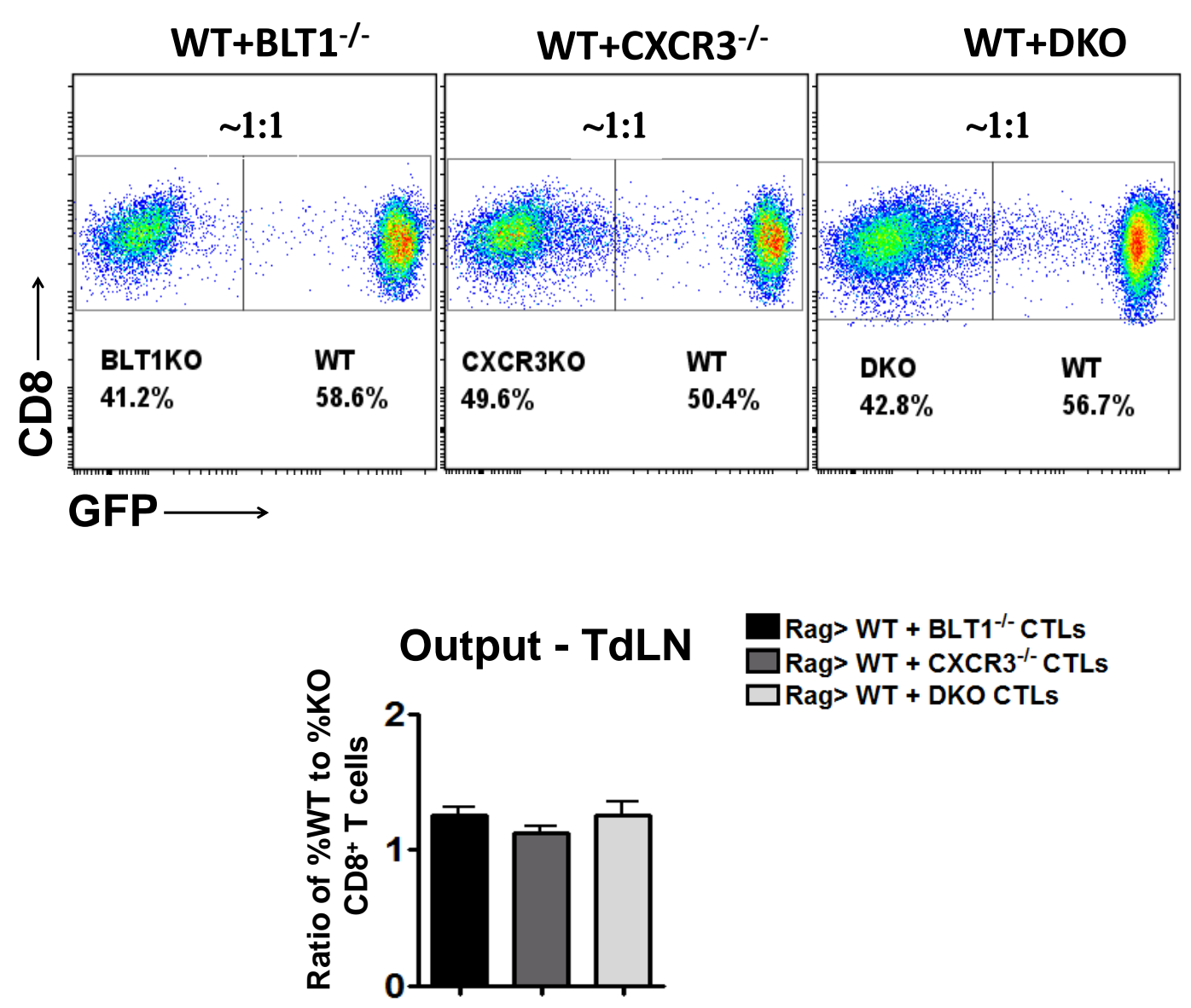

Figure 22: Equal proportions of WT and knockout $\mathrm{CD}^{+} \mathrm{T}$ cells in the TdLN of $\mathrm{Rag2}^{-/-}$mice. Co-transfer experiments were performed as mentioned in methods. Briefly, $\mathrm{CD}^{+} \mathrm{T}$ cells were isolated from tumor bearing $(3-5 \mathrm{~mm})$ WT(GFP), BLT1 ${ }^{-/}$(non GFP), $\mathrm{CXCR}^{-/}$(non-GFP) mice and $\mathrm{BLT}^{-/} \mathrm{CXCR}^{-/}$ (non-GFP) mice. WT and $\mathrm{KO} \mathrm{CD} 8^{+} \mathrm{T}$ cells were equally mixed and 1 million cells were injected i.v. in tumor bearing Rag2 ${ }^{-/-}$mice. Animals were sacrificed when the tumors reached $7-8 \mathrm{~mm}$ tumor diameter. \%WT and $\mathrm{KO} C D 8^{+} \mathrm{T}$ cells were determined upon gating on live $\mathrm{CD}^{+} \mathrm{CD}^{+} \mathrm{T}$ cells and looking for $\mathrm{GFP}^{+}$and $\mathrm{GFP}^{-}$ populations respectively. Representative dot plots of \%WT and \%KO CTLs 
obtained from TdLNs of $\mathrm{Rag2}^{-/-}$mice when the tumor reaches $7-8 \mathrm{~mm}$ tumor diameter. Cumulative bar graph demonstrating the ratio of \%WT to \%KO CTLs in TdLN is also shown. Data is representative of two independent experiments for each transferred combination with $n=5$ in each experimental group. 

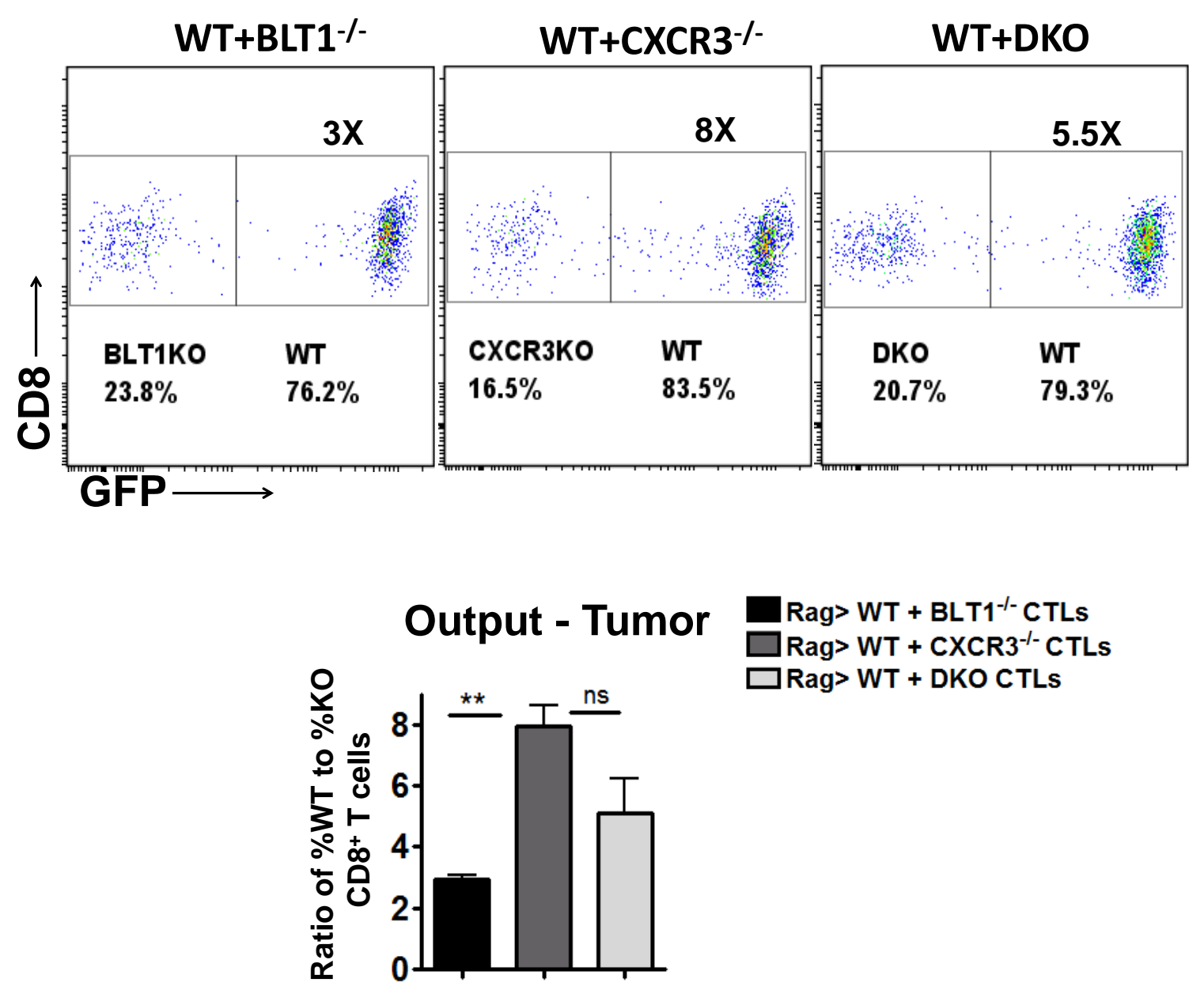

Figure 23: WT CD8 ${ }^{+} \mathrm{T}$ cells do not facilitate knockout $\mathrm{CD}^{+} \mathrm{T}$ cell infiltration to tumors. Co-transfer experiments were performed as mentioned in methods. A) Representative dot plots of \%WT and \%KO CTLs obtained from tumor of $\mathrm{Rag}^{-/}$mice when the tumor reaches $7-8 \mathrm{~mm}$ tumor diameter. The \%GFP+ and \%GFP- is shown after gating on $\mathrm{CD}^{+} \mathrm{T}$ cells. B) Cumulative bar graph demonstrating the ratio of \%WT to \%KO CTLs in TdLN is also shown. C) Cumulative bar graph demonstrating total live $\mathrm{CD}^{+} \mathrm{T}$ cells in tumors of Rag2 $2^{--}$ mice adoptively transferred with equal proportions of WT and knockout CD8 ${ }^{+} \mathrm{T}$ cells. Bar in black represents WT $+B L T 1^{-/-}$CTL mix; grey bars represent WT + 
$\mathrm{CXCR}^{-/-}$CTL mix and light grey bar represents $\mathrm{WT}+\mathrm{DKO}$ CTL mix. Data is representative of two independent experiments for each transferred combination with $n=5$ in each experimental group. 

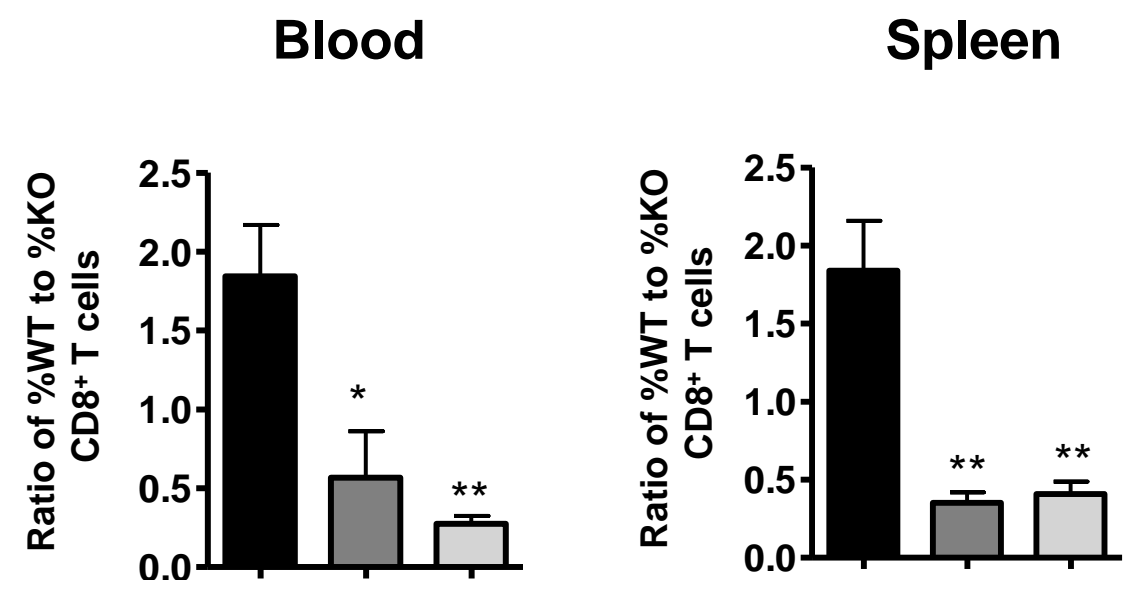

Rag > WT + BLT1 ${ }^{-1-}$ CTLS Rag > WT + CXCR3 ${ }^{-1-}$ CTLs Rag > WT + DKO CTLs

Figure 24: $\mathrm{CXCR3}^{--}$and $\mathrm{DKO} \mathrm{CD}^{+} \mathrm{T}$ cells are significantly more as compared to WT cells in blood and spleen of tumor bearing Rag2 ${ }^{-/-}$mice. Co-transfer experiments were performed as mentioned in methods. Briefly, CD8 ${ }^{+}$ T cells were isolated from tumor bearing (3-5mm) WT(GFP), BLT1 ${ }^{-/}$(non GFP), $\mathrm{CXCR}^{-/}$(non-GFP) mice and $\mathrm{BLT}^{-{ }^{-} \mathrm{CXCR}{ }^{-/}}$(non-GFP) mice. WT and KO $\mathrm{CD}^{+} \mathrm{T}$ cells were equally mixed and 1 million cells were injected i.v. in tumor bearing Rag2 ${ }^{-/}$mice. Animals were sacrificed when the tumors reached $7-8 \mathrm{~mm}$ tumor diameter. \%WT and $\mathrm{KO} \mathrm{CD} 8^{+} \mathrm{T}$ cells were determined upon gating on live $\mathrm{CD}^{+} \mathrm{CD}^{+} \mathrm{T}$ cells and looking for $\mathrm{GFP}^{+}$and $\mathrm{GFP}^{-}$populations respectively. Cumulative bar graph representing ratio of \%WT to \%KO CTLs (frequency of $\mathrm{CD}^{+} \mathrm{T}$ cells) is shown. Data representative of two independent experiments with $\mathrm{n}=5$ per group in each experiment. 
CHAPTER VI

\title{
PD-1 BLOCKADE BASED VACCINE FAILS IN BLT1 ${ }^{--}$, $\mathrm{CXCR3}^{-/-}$AND BLT1 ${ }^{-/-}$ $\mathrm{CXCR}^{-/-} \mathrm{MICE}$
}

\begin{abstract}
INTRODUCTION
Data from previous chapters demonstrated the role of BLT1 and CXCR3 receptor in regulating endogenous anti-tumor responses. In this chapter we sought to investigate the role of $\mathrm{BLT1}$ and $\mathrm{CXCR3}$ receptors in regulating a vaccineinduced immune response. Whether BLT1 and CXCR3 receptor mediated CTL recruitment to tumors and anti-tumor immunity could be bypassed under the presence of an external immune stimulation was tested. We tested PD-1 blockade based immunotherapy to answer this question.

PD-1 is an immunosuppressive molecule present on $\mathrm{T}$ cells that have been activated against an antigen. PD-1 based immunosuppression is involved in restraining or attenuating an inflammatory response after an infection or cause for inflammation has been eliminated. However, in the context of anti-tumor immunity, as discussed before, PD-L1-PD-1 based immunosuppression leads to tumor mediated immunosuppression of CTLs enabling tumor immune evasion. Blocking PD-1 expression on $\mathrm{T}$ cells enhances anti-tumor immunity and has recently revolutionized the field of immunotherapy.
\end{abstract}


In order to conduct this experiment, we employed PD-1 antagonistic antibody based vaccine formulation consisting of Trp-2 melanoma peptide. The vaccine was administered as a therapeutic regimen upon tumor challenge. The data presented here indicate an obligate requirement for $\mathrm{BLT1}$ and $\mathrm{CXCR} 3$ receptors in obtaining vaccine efficacy. CTL infiltration studies were carried out in tumors, spleen, TdLN and blood of unvaccinated and vaccinated mice. This chapter would provide essential information on the requirement of BLT1 and CXCR3 pathways in mediating efficacy of PD-1 blockade based immunotherapy. 
RESULTS

\section{Obligate requirement for BLT1 and CXCR3 in anti-PD-1 Ab based vaccine efficacy}

The importance of BLT1 and CXCR3 in vaccine induced anti-tumor immune

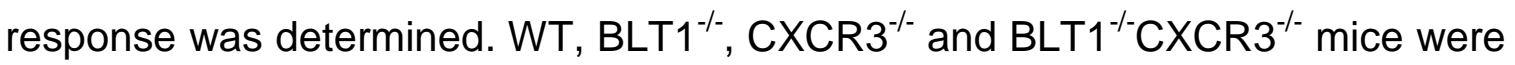
challenged with $10^{5}$ B16 cells subcutaneously on Day 0 followed by vaccine administration on day +5 and day +15 . Although the vaccine did not completely eradicate the tumors in WT mice, there was a significant reduction in the tumor

growth kinetics in WT mice upon vaccination. However, the vaccine completely failed to delay tumor growth in the $\mathrm{BLT}^{-1}{ }^{-}, \mathrm{CXCR}^{-/}$and $\mathrm{BLT} 1^{-1-} \mathrm{CXCR}^{-/-}$animals (Figure 25).

The vaccine efficacy correlated with $\mathrm{CD}^{+} \mathrm{T}$ cell infiltration to tumors, with significantly reduced $\mathrm{CD}^{+} \mathrm{T}$ cell infiltration in tumors of knockout mice upon vaccination. As expected the vaccine decreased $\mathrm{CD}^{+} \mathrm{T}$ cell numbers in the blood of WT mice as a reflection of the concurrent increase in the $\mathrm{CD} 8^{+} \mathrm{T}$ cells in tumors of WT mice (Figure 26). The percentages of $\mathrm{CD} 8^{+} \mathrm{T}$ cells in the TdLNs and spleens of the knockout animals were comparable to the WT animals in both the vaccinated and unvaccinated cohorts (Figure 27). The ability of the vaccine to enhance $\mathrm{CD} 8^{+} \mathrm{T}$ cell migration in WT tumors but not the knockout tumors was also confirmed by confocal microscopy (Figure 27 A). Granzyme-B an effector molecule secreted by $\mathrm{CD} 8^{+} \mathrm{T}$ cells was significantly enhanced in WT mice upon vaccination, the increase was absent in knockout animals upon vaccination 
suggesting reduction in effector responses in tumors of knockout mice with or without vaccination (Figure 27 B). $C D 8^{+} T$ cells derived from blood and TdLN of tumor bearing mice showed increased BLT1 expression (increased mean fluorescent intensity) and a moderate CXCR3 upregulation (more counts of cells expressing CXCR3) upon vaccination (Figure 28A and 28 B). These results suggest that optimum efficacy of anti-PD-1 antibody based vaccine requires the expression of both BLT1 and CXCR3 for effective CTL infiltration to tumors and anti-tumor immunity. 


\section{Therapeutic Vaccine Design}
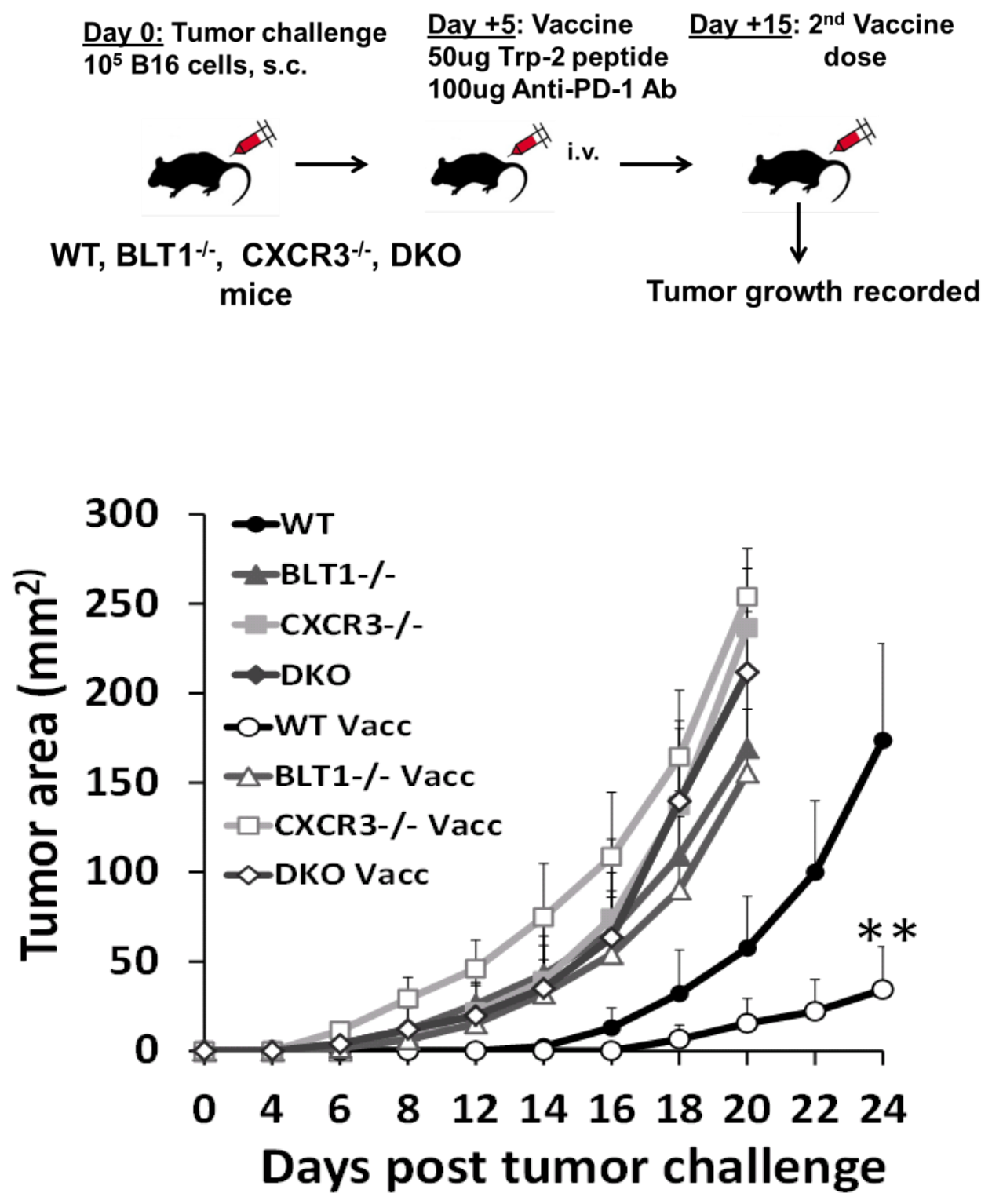

Figure 25: Obligate requirement of BLT1 and CXCR3 for optimum efficacy of anti-PD-1 antibody based immunotherapy. WT (Unvacc: $n=4$, Vacc: $n=6$ ), BLT1 $^{--}$(Unvacc: $n=5$, Vacc: $\left.n=5\right), \mathrm{CXCR}^{-/}$(Unvacc: $n=3$, Vacc: $\left.n=5\right)$ and DKO (Unvacc: $n=6$, Vacc: $n=5$ ) mice were subcutaneously challenged with $10^{5}$ B16 cells and left either unvaccinated (PBS) or vaccinated with Trp-2 peptide (50 $\mu \mathrm{g})$ 
and anti-PD-1 Ab $(100 \mu \mathrm{g})$ twice intravenously on day +5 and +15 post tumor inoculation. Tumor area measured by multiplication of two perpendicular diameters in unvaccinated and vaccinated $\mathrm{WT}, \mathrm{BLT}^{-{ }^{--}}, \mathrm{CXCR}^{-{ }^{--}}$and DKO mice is shown. Data is representative of two independent experiments. 
A

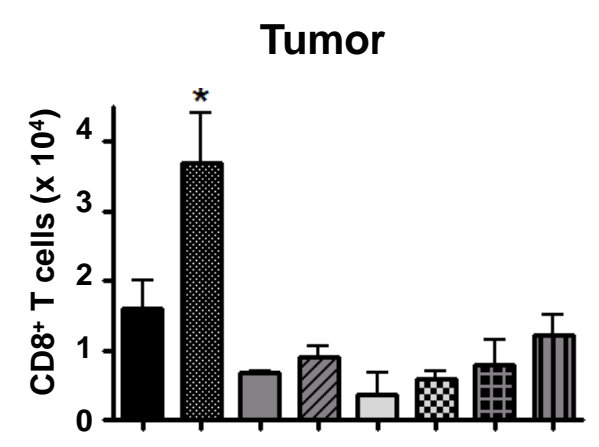

C

TdLN

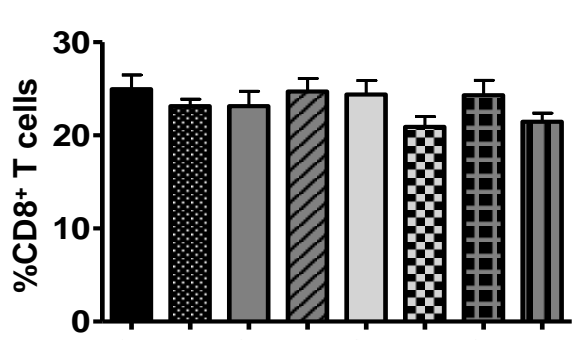

B

Blood

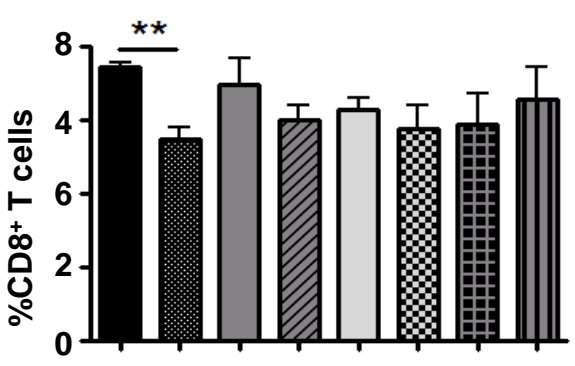

D

Figure 26: Vaccine enhances $\mathrm{CD}^{+} \mathrm{T}$ cell infiltration in tumors of WT mice but not knockout mice. WT (Unvacc: $n=4$, Vacc: $n=6$ ), BLT1 ${ }^{-/}$(Unvacc: $n=5$, Vacc: $n=5$ ), CXCR3 ${ }^{-/}$(Unvacc: $n=3$, Vacc: $n=5$ ) and DKO (Unvacc: $n=6$, Vacc: $\mathrm{n}=5$ ) mice were subcutaneously challenged with $10^{5}$ B16 cells and left either unvaccinated (PBS) or vaccinated with Trp-2 peptide $(50 \mu \mathrm{g})$ and anti-PD-1 Ab $(100 \mu \mathrm{g})$ twice intravenously on day +5 and +15 post tumor inoculation. A. Enhanced $\mathrm{CD}^{+} \mathrm{T}$ cell numbers in tumors of WT mice but not knockout mice upon vaccination. Cumulative bar graph representing $\mathrm{CD} 8^{+} \mathrm{T}$ cell numbers per million total tumor cells (frequency of total) in unvaccinated and vaccinated WT, 
$\mathrm{BLT1}^{-/}, \mathrm{CXCR}^{-/-}$and DKO mice is represented. B,C. Cumulative bar graph representing \%CD8 ${ }^{+} \mathrm{T}$ cells (frequency of $\mathrm{CD} 45^{+}$cells) in spleen (B) and TdLN (C) of unvaccinated and vaccinated $\mathrm{WT}, \mathrm{BLT1}^{-/}, \mathrm{CXCR}^{-/}$and DKO mice is shown. D. Cumulative bar graph representing $\% \mathrm{CD} 8^{+} \mathrm{T}$ cells (frequency of $\mathrm{CD}_{4} 5^{+}$cells) in blood of unvaccinated and vaccinated $\mathrm{WT}, \mathrm{BLT} 1^{-1}, \mathrm{CXCR}^{-/-}$and DKO mice is shown. Data is representative of two independent experiments. 

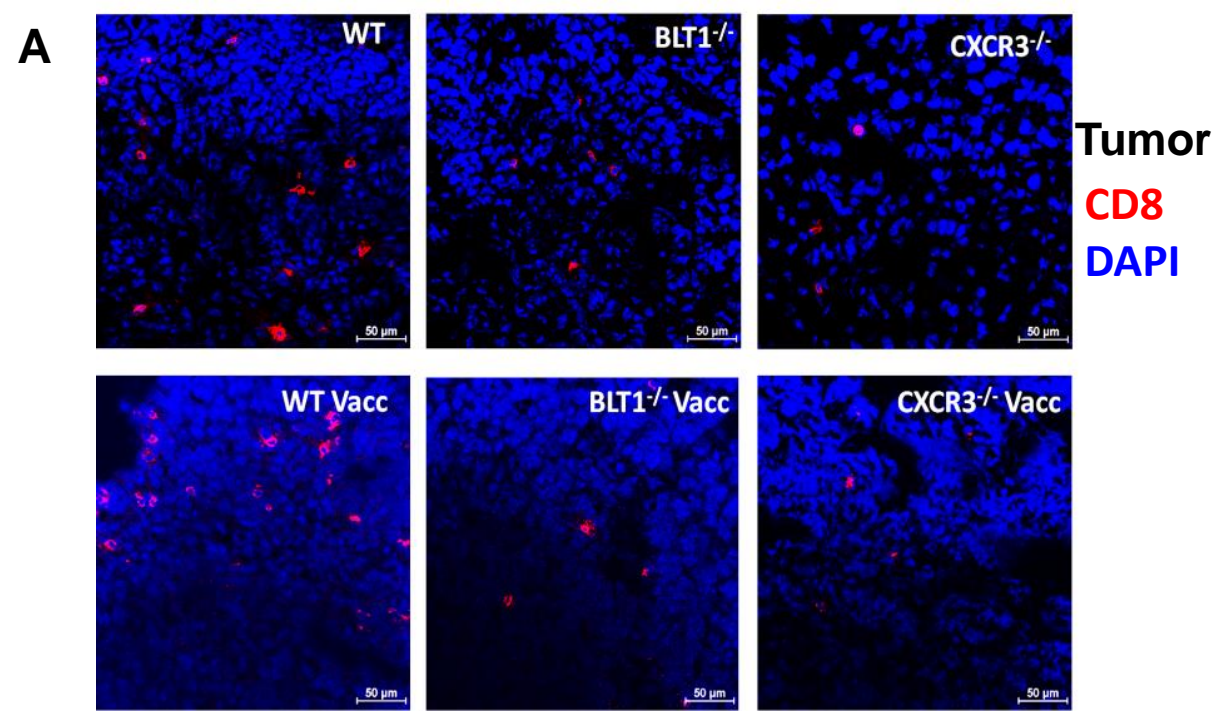

B
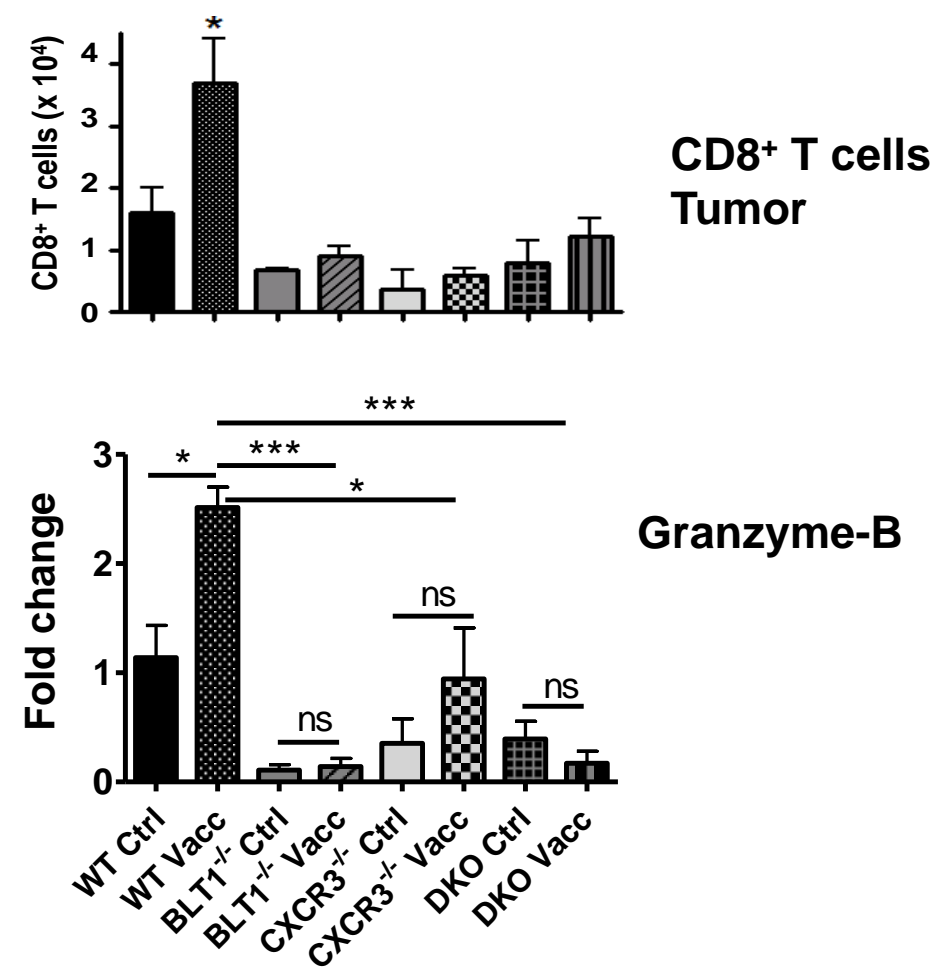

Figure 27: Vaccine enhances $\mathrm{CD}^{+} \mathrm{T}$ cell infiltration in tumors of WT mice but not knockout mice. A) Representative immunofluorescence staining images of $\mathrm{CD}^{+} \mathrm{T}$ cells in $\mathrm{WT}, \mathrm{BLT1}^{-/-}$and $\mathrm{CXCR}^{-/-}$tumors from vaccinated or unvaccinated mice. Tumors harvested were frozen, sectioned and stained as 
described in Methods, CD8 represented in Red, DAPI in blue. The images were captured using Nikon A1R confocal microscope. The scale represents $50 \mu \mathrm{M}$. Data representative of two independent experiments. B) Enhanced $\mathrm{CD} 8^{+} \mathrm{T}$ cell numbers in tumors of WT mice but not knockout mice upon vaccination. Cumulative bar graph representing $\mathrm{CD}^{+} \mathrm{T}$ cell numbers per million total tumor cells (frequency of total) in unvaccinated and vaccinated WT, BLT1 $1^{-1-}$, CXCR3 $3^{-1-}$ and DKO mice is represented. Also, fold change of granzyme-B transcript in RNA isolated from $\mathrm{WT}, \mathrm{BLT}^{-/-}$and $\mathrm{CXCR}^{-/}$tumors of vaccinated and unvaccinated mice when the knockout tumors reach $15 \mathrm{~mm}$ tumor diameter is shown. GAPDH was used as the housekeeping gene. Relative fold change to WT tumors is shown. $n=4$ in each group. 
A

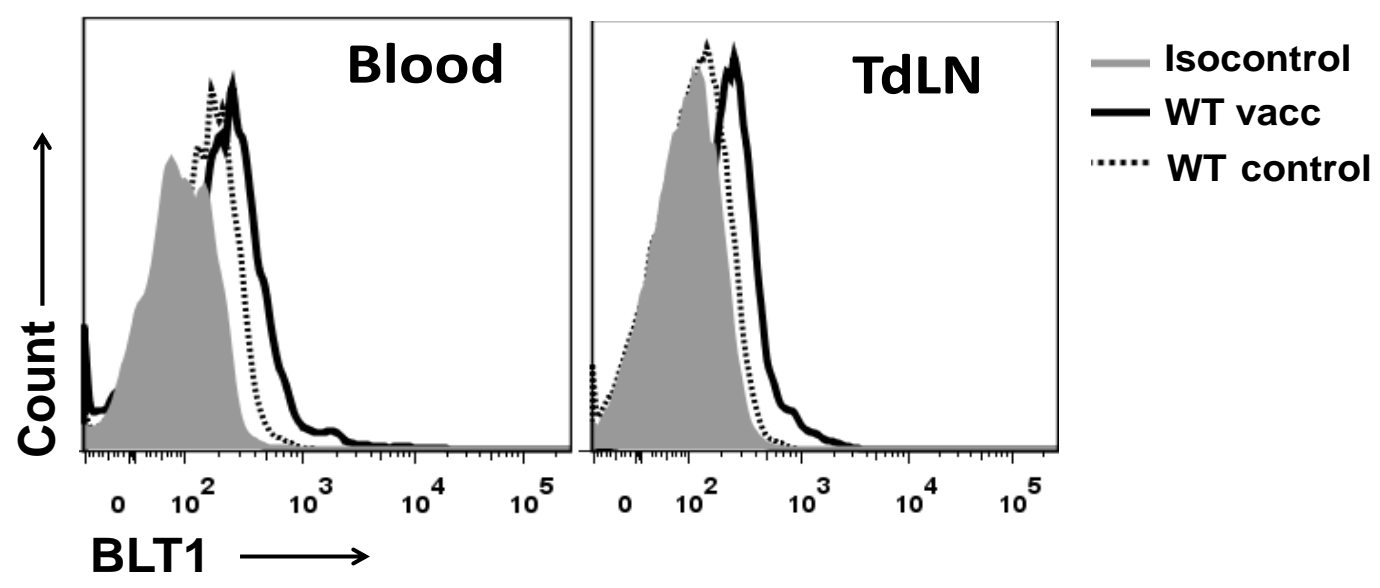

B

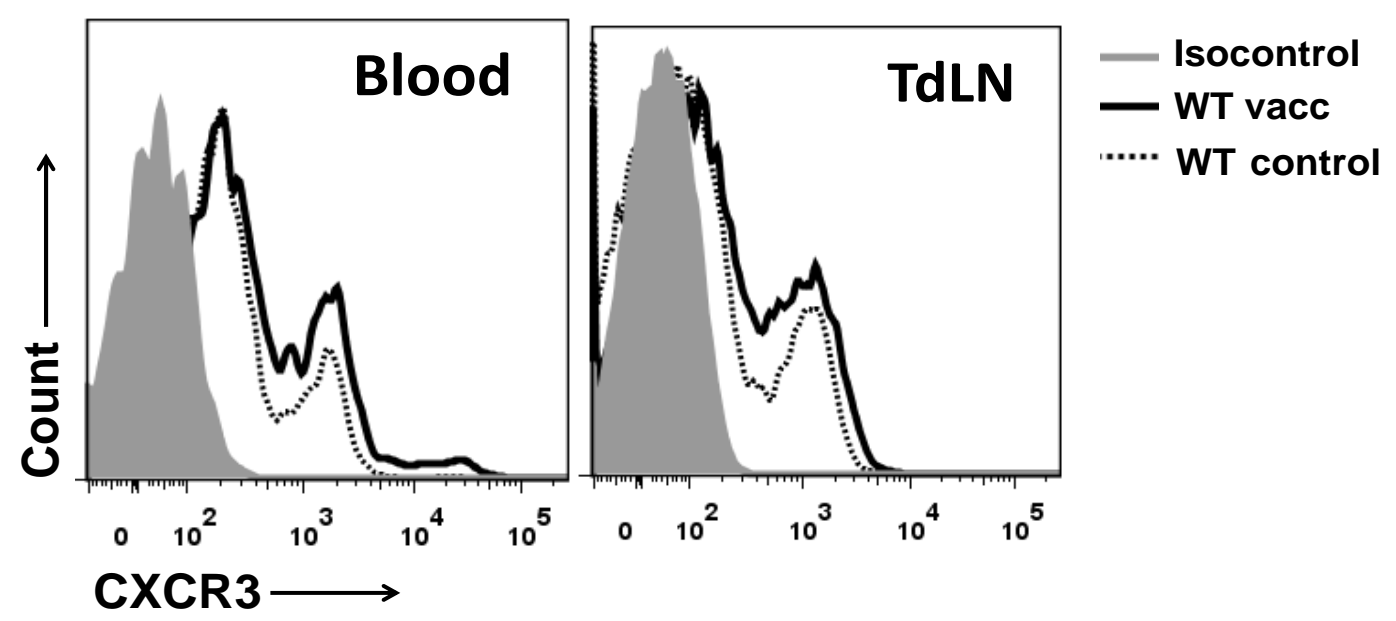

Figure 28: Enhanced expression of BLT1 and CXCR3 on $\mathrm{CD}^{+} \mathrm{T}$ cells upon vaccination. $\mathrm{CD}^{+} \mathrm{T}$ cells from blood and tumor draining lymph nodes of unvaccinated and vaccinated tumor bearing WT mice were analyzed for BLT1 Antibody developed in the lab (unpublished study) and CXCR3 as mentioned in the methods section. $n=5$ in each group. A) Representative histogram plots for BLT1 expression on $\mathrm{CD} 8^{+} \mathrm{T}$ cells (gated on $\mathrm{CD} 3^{+} \mathrm{CD} 8^{+} \mathrm{T}$ cells) from blood and 
TdLN of WT vaccinated and unvaccinated control mice. Dotted line represents WT unvaccinated $\mathrm{CD}^{+} \mathrm{T}$ cells and solid black line represents WT vaccinated $\mathrm{CD}^{+} \mathrm{T}$ cells. B) Representative histogram plots for CXCR3 expression on $\mathrm{CD}^{+}$ T cells (gated on $\mathrm{CD}^{+}{ }^{+} \mathrm{CD} 8^{+} \mathrm{T}$ cells) from blood and TdLN of WT vaccinated and unvaccinated control mice. Dotted line represents WT unvaccinated $C D 8^{+} \mathrm{T}$ cells and solid black line represents WT vaccinated $\mathrm{CD} 8^{+} \mathrm{T}$ cells. 


\section{CHAPTER VII}

\section{DISCUSSION}

Cancer immunotherapies rely on achieving stronger and long lasting effector $\mathrm{CD}^{+} \mathrm{T}$ cell responses in the tumor. A major obstacle for attaining this goal is the inefficient migration of $\mathrm{CD}^{+} \mathrm{T}$ cells into the tumor $[40,75,144]$. The results presented here suggest that both BLT1 and CXCR3 are independently required for $\mathrm{CD}^{+} \mathrm{T}$ cell migration to tumors and sustained anti-tumor immunity. In the absence of either of these receptors, there is a breach in achieving an effective anti-tumor response.

Although BLT1 is expressed on a variety of leukocytes, there is a preferential BLT1 mediated recruitment of certain cells under specific disease condition. For example, Th2 and $\mathrm{CD}^{+} \mathrm{T}$ cells cell infiltration is preferred during airway hyperresponsiveness in asthma, T cells in autoimmune uveitis, macrophages in diet induced obesity and atherosclerosis and neutrophils in silica induced lung cancer promotion; in all of these models genetic deletion of BLT1 was shown to be protective [181, 183, 186, 189-192]. Recently, we demonstrated a crucial antitumor role of $\mathrm{BLT} 1$ in mediating $\mathrm{CD} 8^{+} \mathrm{T}$ cell recruitment to tumors using $\mathrm{TC}-1$ cervical cancer model wherein BLT1 deficient mice showed enhanced tumor growth and reduced survival compared to WT mice [176]. Lack of CTL infiltration to tumors can delay an anti-tumor response in other types of cancers as well. 
Using an autologous melanoma tumor model expressing self-antigens [193], herein we corroborated that BLT1 mediates CTL recruitment to tumors and thus plays an important role in anti-tumor immunity; consistent with the TC-1 cervical cancer model. This suggests that BLT1 mediated regulation of CTL infiltration may be true across a variety of immunogenic solid tumor types. Hence, BLT1 mediated recruitment of various immune cells at specific locations in different tumor models is key to the type of inflammation (pro-tumor or anti-tumor) that accrues. In melanoma, BLT1 mediated $\mathrm{CD}^{+} \mathrm{T}$ cell infiltration to tumors is a crucial mechanism for effective anti-tumor immunity. Consistent with this observation, anti-tumor response in spontaneous $\mathrm{Apc}^{\mathrm{Min} /}{ }^{+}$intestinal model of tumorigenesis also requires the expression of BLT1 (unpublished data).

Previous studies have reported a pro-tumorigenic role for BLT1-LTB 4 pathway, in cancer [187, 194-197]. Our studies highlight the importance of BLT1 on immune cells (CTLs) in achieving an effective anti-tumor response. Moreover, the data presented here suggests that in the absence of BLT1, major CTL chemokine receptors like CXCR3 that have been demonstrated to be indispensable for $\mathrm{T}$ cell trafficking at the tumor vasculature [142], cannot achieve optimum CTL infiltration to tumors.

In a GM-CSF gene transduced leukemia model, Yokota et.al. showed that BLT1 ${ }^{-1-}$ mice showed similar primary anti-tumor response but enhanced recall memory response. The better recall response induced by $\mathrm{GM}-\mathrm{CSF}$ in $\mathrm{BLT} 1^{-1-}$ mice was attributed to enhanced DC maturation and function, reduced MDSC numbers, 
enhanced NK cells in the knockout tumors and an enhanced Th1 and predominantly skewed Th2 response. In contrast to this crucial finding, our results suggest that $\mathrm{BLT1} 1^{-/}$mice have a significant defect in primary anti-tumor response due to defective $\mathrm{CD}^{+} \mathrm{T}$ cell infiltration in the tumors of $\mathrm{BLT}^{-/-}$mice; the other immune cell subsets remaining similar to those in WT mice. We also did not find any difference in the cytotoxic function in $\mathrm{BLT}^{-/}$mice as evident through in vivo-killing assay by immunization using peptide as well as tumor cells (data not shown). The divergence in the results observed can be accounted to the differences in the mouse strains (BALB/c) and induction of GM-CSF in the tumor cells [187]. GM-CSF is long known to induce DC maturation and T helper responses [198]. Also, the BALB/c mice model has been reported to be biased towards Th2 response [199] and Th2 responses are crucial for memory antitumor responses [200]. Although we did not find any difference in the numbers of $\mathrm{CD}^{+} \mathrm{T}$ cells, NK cells or myeloid cells in the tumors of $\mathrm{BLT}^{-/}$mice, the helper contributions of $\mathrm{CD}^{+} \mathrm{T}$ cells and NK cells in $\mathrm{CD}^{+} \mathrm{T}$ cell mediated anti-tumor immunity cannot be ruled out. Further studies are essential to determine the role of $\mathrm{LTB}_{4}-\mathrm{BLT} 1$ pathway in $\mathrm{CD} 4^{+} \mathrm{T}$ cell dependent primary and memory anti-tumor responses.

The role of CXCR3 and the ligands CXCL9 and CXCL10 in anti-tumor immunity is well established $[81,142,143,201]$. In a recent study, Mikuchi et.al elegantly demonstrated that CXCR3 mediated signalling to be a critical and an indispensable checkpoint for tumor antigen specific $\mathrm{CD} 8^{+} \mathrm{T}$ cells to traffic across the tumor vasculature for carrying out effective tumoricidal activity in mice and 
human melanoma [142]. CTL chemokine receptors CCR5 and CCR2 were not essential for CXCR3 mediated CTL extravasation across tumor vessels despite the presence of CCL2 and CCL5 chemokines in the tumor mileu. Using an antigen specific B16Ova - OT-I system and ACT setting, they showed that WT OT-I but not $\mathrm{CXCR}^{-/-}$OT-I CTLs were able to significantly reduce tumors; with $50 \%$ of WT OT-I transferred mice showing complete tumor regression. Consistent with their study, our data from tumor kinetics in WT versus $\mathrm{CXCR}^{-/-}$ mice and adoptive transfer of tumor educated WT or CXCR3 ${ }^{-}$CTLs demonstrated a crucial indispensable role for CXCR3 in mediating CTL recruitment to tumors and endogenous anti-tumor immunity. Moreover, in a therapeutic vaccine model based on PD-1 blockade in WT or $\mathrm{CXCR}^{-/-}$tumor bearing mice suggested an obligate role for CXCR3 in the success of immune checkpoint blockade based therapies. Additionally, we demonstrated an equally indispensable role for BLT1 for endogenous anti-tumor immunity and therapeutic efficacy. Mikucki et. al. demonstrated an essential role for CXCR3 in mediating firm adhesion of tumor $\mathrm{Ag}$ specific $\mathrm{CD}^{+} \mathrm{T}$ cells at the tumor vessels while not affecting the rolling property of the CTLs. However, at what juncture of the multistep trafficking process is $\mathrm{BLT1}$ on $\mathrm{CD} 8^{+} \mathrm{T}$ cells required for homing into tumors, remains to be determined.

With respect to the functionality of the $C D 8^{+} \mathrm{T}$ cells in the tumor microenvironment, CXCR3 expression plays a crucial role in interferon gamma secretion as $\mathrm{CXCR}^{-1-}$ CTLs in the tumors have a defect in interferon gamma production. Various other studies have shown similar defects in IFNy production 
of $\mathrm{CXCR3}^{-/}$T cells $[89,202,203]$. Perturbed amplification loop in IFNY production due to reduced Th1 and NK cells in $\mathrm{CXCR}^{-/-}$or increased suppressive function of $\mathrm{M} 2$ macrophages in $\mathrm{CXCR}^{-/-}$mice may be the reasons for defective IFNy production in $\mathrm{CXCR}^{-/-} \mathrm{CD}^{+} \mathrm{T}$ cells $[92,204]$. Another explanation of perturbed IFNy in $\mathrm{CXCR}^{-/-}$CTLs in tumor microenvironment may be due to significantly reduced $\mathrm{CD} 4^{+} \mathrm{T}$ cells, NK cells and IL-2 (data not shown) in those tumors. CD4 ${ }^{+} \mathrm{T}$ cells support $\mathrm{CD} 8^{+} \mathrm{T}$ cell responses not only by IL-2, production which is required for survival and expansion of effector $\mathrm{T}$ cells but also by licensing of APCs [205]. NK cells are also a crucial part of anti-tumor immunity. NK cells by secreting IL-2, IFNy, CXCL10 aids in the maintenance of T-helper and CTL population [206]. It is also conceivable that due to less IL-2 production in the tumors, the survival and effector functions of the $\mathrm{CXCR}^{-/-} \mathrm{CTLS}$ cells is affected. Importantly, CXCR3 is a major player in the recruitment of T cells and NK cells and their survival and effector functions in the tumors. A recent study demonstrated that adenosine in the tumor milieu suppressed the production of CXCL10 followed by suppression in T cell infiltration; and partial reversion was seen upon adenosine receptor blockade [143]. Another study suggested that adenosine receptor blockade therapy failed to reduce tumor growth in $\mathrm{CXCR3}^{-/-}$mice [207]. Hence, expression of CXCR3 on CTLs in the tumor seems crucial for CTL effector function. Also, it is essential to understand the roles of BLT1 and CXCR3 on $\mathrm{CD} 4^{+} \mathrm{T}$ cells and NK cells as well as their crosstalk with $\mathrm{CD} 8^{+} \mathrm{T}$ cell survival and effector functions. 
In general various leukocyte subtypes express several chemo-attractant receptors in overlapping patterns (eg. BLT1 is expressed on both activated T cells and myeloid cells) and one cell-type express various receptors that can respond to multiple chemo-attractant cues that may be present at the target tissue. Using a simplified 2D agarose based system where neutrophils encountered two different chemoattractant signals in a defined spatial array, neutrophils were shown to chemotax sequentially, first migrating up a primary gradient of IL-8 into a disorienting concentration which later could effectively retain capacity to resume migration to a secondary distant chemoattractant gradient of $\mathrm{LTB}_{4}$, suggesting a potential for step-by-step navigation of immune cells from one chemo-attractant gradient to another in complex chemo-attractant fields [195]. Hence, two attractant pathways specific for the same cell may function together rather than being redundant in order to effectively recruit immune cells. Our studies suggest that BLT1 and CXCR3 seem to play an essential, non-redundant, cell-autonomous role in $\mathrm{CD}^{+} \mathrm{T}$ cell infiltration to tumors and anti-tumor immunity.

We attempted to study the combinatorial regulation of CTL infiltration to tumors by BLT1 and CXCR3 via generation of BLT1/CXCR3 double knockout mice (DKO). The data presented here suggest a lack of synergism in BLT1 and CXCR3 but a probable occurrence of interdependence since there was no further enhancement of tumor growth in the DKO mice compared to either of the single knockout mice. Studies with the model of arthritis revealed that BLT1 mediated infiltration of WT neutrophils in $\mathrm{BLT} 1^{-/}$mice facilitated infiltration of endogenous 
$\mathrm{BLT1}^{-/}$neutrophils to the inflamed joint suggesting that BLT1 expression on neutrophils is essential only for the initial recruitment and other chemokines could then perpetuate the disease progression [180]. However, our co-transfer experiments with WT and individual knockout CTLs revealed that WT CTLs do not facilitate additional $\mathrm{BLT}^{-/}$or $\mathrm{CXCR}^{-/-} \mathrm{CTL}$ infiltration to tumors suggesting that BLT1 and CXCR3 mediated signalling cannot be bypassed by other chemoattractant systems for CTL migration in to tumors.

The magnitude of importance of BLT1 and CXCR3 signalling pathways in vaccine-induced immune response was tested using immune-checkpoint blockade based vaccine. Blockade of Programmed cell Death-1 (PD-1) pathway has been recently FDA approved and is a promising anti-tumor immunotherapy in humans as it releases the brakes on the $T$ cells thereby enhancing their function [208-211]. Anti-PD-1 antibody therapy was shown to enhance T cell infiltration of adoptively transferred $T$ cells [211]. The data presented herein shows that while anti-PD-1 based vaccine enhanced T cell infiltration to tumors thereby reducing tumor growth in WT mice; the vaccine lost its efficacy in the absence of either or both BLT1 and CXCR3 receptors, a phenotype ascribed to the failure of the knockout CTLs to infiltrate the tumors. We hereby show an obligate role for both BLT1 and CXCR3 expression on CTLs in achieving optimum anti-PD-1 based vaccine efficacy.

In lung metastatic melanoma model, melanoma cells were shown to be a source for CXCL9 and CXCL10 production. Among the immune cells, CD4 ${ }^{+} \mathrm{T}$ cells were considered the major producers of CXCL9 as well as IFNy in the metastatic 
nodules in lung [143]. While most myeloid cells can readily make $\mathrm{LTB}_{4}$, the source of this BLT1 ligand in the B16 tumors remain to be determined.

Taken together, these findings suggest potential ways to improve the current ACT therapies, including Chimeric Antigen Receptor (CAR) based therapies by employing BLT1 as well as CXCR3 receptor up-regulation strategies on tumor $\mathrm{Ag}$ specific $\mathrm{CD}^{+} \mathrm{T}$ cells or receptor specific agonists to facilitate increased CTL trafficking into tumors. Since studies show that the cells used for ACT already have considerable CXCR3 expression on them [142], BLT1 can be upregulated on these cells to achieve better infiltration. Alternatively enhancing the CXCR3 chemokine levels as well as $\mathrm{LTB}_{4}$ levels in the tumor may help achieve increased CTL infiltration to tumors. These findings also have an important implication in probing the potential efficacy of PD-1 blockade based treatment in patients bearing CXCR3 receptor polymorphisms (for e.g. CXCR3rs2280964), which results in an altered receptor that fails to be expressed on cell surface [212]. Taken together, our studies suggest that, in melanoma tumor, LTB $_{4}-\mathrm{BLT1}$ pathway, is equally essential as CXCL9/CXCL10 - CXCR3 for CTL migration to tumors and anti-tumor immunity and can be targeted for therapy. 


\section{SUMMARY}

Immunotherapies have revolutionized the field of cancer therapy and have shown considerable efficacy in the clinic. However, the efficacy of the current immunotherapy is restricted to a few patients while many patients remain unresponsive to treatment. A major obstacle recognized only recently in the success of these immunotherapies is defective $\mathrm{CD} 8^{+} \mathrm{T}$ cell recruitment to tumors which significantly impairs the anti-tumor response. Chemokine-chemokine receptor signaling is a crucial T cell homing mechanism. Herein, we investigated the role of leukotriene $\mathrm{B}_{4}$ receptor BLT1 and CXCL9 and CXCL10 receptor CXCR3 in regulating $\mathrm{CD}^{+} \mathrm{T}$ cell migration and anti-tumor immunity using an autologous B16 melanoma model.

The results in this thesis suggest an important role for chemoattractant receptors BLT1 and CXCR3 in anti-tumor immune regulation (Figure 29). Both $\mathrm{BLT}^{-/}$and $\mathrm{CXCR}^{-/-}$mice demonstrated significantly enhanced tumor growth to a similar extent that reduced survival compared to WT mice (Figure 9). Investigating cellular mechanisms to this phenotype revealed that BLT1 and CXCR3 both are essential for $\mathrm{CD}^{+} \mathrm{T}$ cell migration to tumors, myeloid cell infiltration levels remaining similar (Figure 10). Analysis of the effector functions of WT and knockout $\mathrm{CD}^{+} \mathrm{T}$ cells revealed no intrinsic defect in killing ability, IFNy and TNF $\alpha$ secretion in the periphery (Figures 15 and 6). However, compared to WT CTLs from tumors, CXCR3 deficient CTLs had a significant defect in IFNy levels (Figure 16). This suggests that CXCR3 but not BLT1 signaling is essential in the tumor microenvironment for IFNy production. Using adoptive transfer model, the 
data demonstrated that tumor educated WT but not BLT1 and CXCR3 deficient $\mathrm{CD}^{+} \mathrm{T}$ cells reduced tumor growth significantly in Rag2 $2^{--}$mice. In fact the control Rag2 $^{-/-}$mice without any transferred CTLs had similar tumor growth kinetics as mice transferred with either of the knockout CTLs. The tumor growth in mice that received BLT1 and CXCR3 deficient cells correlated with defective tumoral but not TdLN CD8 ${ }^{+} \mathrm{T}$ cell infiltration. This reinforced the importance of both BLT1 and $\mathrm{CXCR} 3$ receptor signaling in $\mathrm{T}$ cell recruitment into tumors (Figure17 and 18).

Analysis of tumor growth in BLT1-CXCR3 double knockout mice suggested similar tumor kinetics between $\mathrm{BLT}^{-/-}, \mathrm{CXCR}^{-/-}$and $\mathrm{BLT}^{-/} \mathrm{CXCR}^{-/}$mice indicating a lack of synergism in regulation of anti-tumor immunity (Figure 21). The possible inter-dependence was determined by co-transfer of WT with either of the knockout $\mathrm{CD}^{+} \mathrm{T}$ cells in tumor bearing Rag2 ${ }^{-/-}$mice. The results obtained suggested that WT CTLs did not facilitate additional $\mathrm{BLT}^{-1^{-}}$, CXCR3 ${ }^{-/-}$or $\mathrm{BLT}^{-1^{-}}$ $\mathrm{CXCR3}^{-/-} \mathrm{CTLs}$ into tumors (Figure 24).

We next sought to investigate the immunotherapy induced responses in $\mathrm{BLT1} 1^{-/}$, $\mathrm{CXCR}^{-/-}$and $\mathrm{BLT}^{-/}{ }^{-\mathrm{CXCR}^{-/-}}$mice. Interestingly, PD-1 blockade based vaccine efficacy was completely abolished in the absence of BLT1 and CXCR3 signaling. TIL analysis of vaccinated and unvaccinated controls suggested that the vaccine enhanced $\mathrm{CD} 8^{+} \mathrm{T}$ cell infiltration into tumors significantly in WT mice but not the knockout mice. The results presented here suggest that BLT1 and CXCR3 mediated anti-tumor immunity cannot be bypassed. This suggests an obligate 
requirement for both BLT1 and CXCR3 in mediating successful checkpoint blockade based vaccine efficacy.

Taken together, the data presented in this thesis suggest an equally crucial role for both BLT1 and CXCR3 receptors for efficient $\mathrm{CD} 8^{+} \mathrm{T}$ cell trafficking to tumors and regulation of endogenous as well as immune checkpoint blockade based vaccine response. The data obtained from tumor growth in double knockout mice and co-transfer experiments indicated that BLT1 and CXCR3 probably are components of a single pathway involved in T cell homing processes. Therefore, ablation of any one of the receptors or both the receptors has similar end result and deficiency in BLT1 is not compensated by CXCR3, making both the genes equally crucial in anti-tumor immunity. 


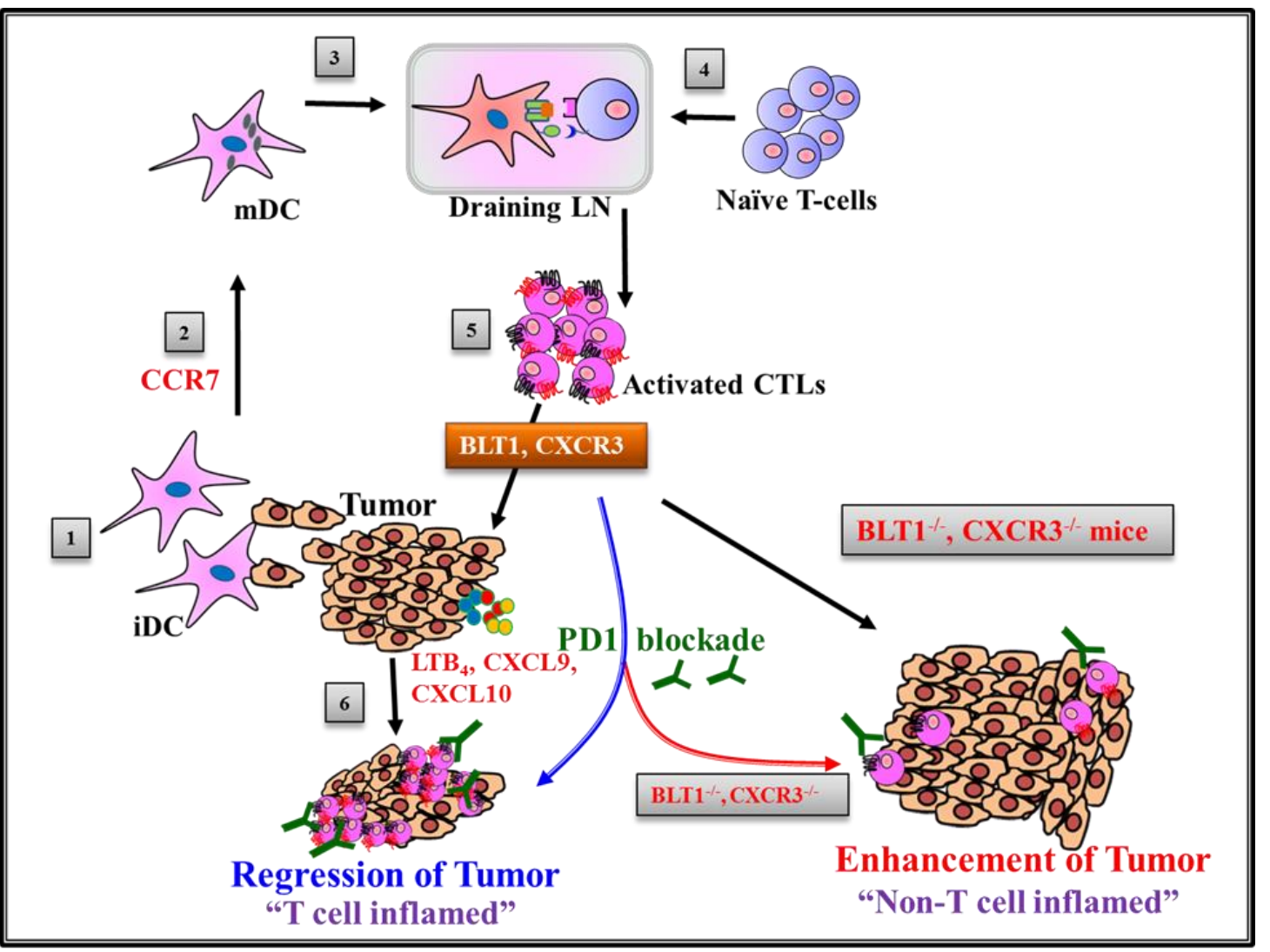

Figure 29: A model for BLT1 and CXCR3 mediated regulation of anti-tumor immunity. DCs phagocytose the tumor cells, undergo maturation and migrate to the tumor draining lymph nodes where they present the tumor antigen to the $T$ cells. The T cells specific for the antigen get activated. Through BLT1 and CXCR3, the activated $\mathrm{CD} 8^{+} \mathrm{T}$ cells then migrate to tumors in response to LTB4 and CXCL9/10 in tumor microenvironment. The present study suggests that BLT1 and CXCR3 are equally important for CTL migration to tumors and antitumor immunity. PD-1 blockade immunotherapy also requires the presence of BLT1 and CXCR3 that gives a "T cell inflamed" phenotype in tumor. In the absence of BLT1 and CXCR3, the PD-1 blockade therapy completely fails and leads to "non- T cell inflamed" phenotype that fails to reduce the tumor size. 


\section{IMPLICATIONS AND FUTURE DIRECTIONS}

The data presented in this thesis suggest an equally crucial role for both BLT1 and $\mathrm{CXCR} 3$ in mediating efficient $\mathrm{CD} 8^{+} \mathrm{T}$ cell migration to melanoma tumors and achieving effective anti-tumor immunity. We demonstrate an important role for $\mathrm{LTB}_{4}-\mathrm{BLT1}$ axis in mediating immune surveillance in both viral Ag derived TC-1 cervical cancer as well as autologous B16 melanoma model, suggesting that BLT1 mediated CTL recruitment to tumors is true probably across various other solid tumors.

Anti-PD-1 antagonistic antibodies, Nivolumab and Pembrolizumab were recently approved by the FDA for treating inoperable end stage melanoma and non-small cell lung carcinoma and have shown promising results in the clinic. Our study has contributed in the understanding of the factors required for the successful efficacy of these therapies. Recent studies have shown that a cohort of patients remain unresponsive to this treatment and that the treatment works best in $\mathrm{T}$ cell inflamed tumor (presence of $T$ cells and $T$ cell chemokines) versus non- $T$ cell inflamed tumor (absence of $\mathrm{T}$ cells and $\mathrm{T}$ cell chemokines). These findings also have an important implication in probing the potential efficacy of PD-1 blockade and possibly even CTLA4 blockade in patients bearing CXCR3 receptor polymorphisms (for e.g. CXCR3rs2280964), which results in an altered receptor that fails to be expressed on cell surface. Also, with respect to BLT1 pathway, it would be crucial to understand if certain patient specific polymorphisms in BLT1 receptor gene or even genes involved in $\mathrm{LTB}_{4}$ production like 5-Lipoxygenase 
and $\mathrm{LTA}_{4}$ hydrolase are associated with unresponsiveness to PD-1 therapies or associated with non-T cell inflamed tumors.

How to redirect the peripheral tumor antigen specific $T$ cells into tumors by targeting chemoattractant-chemoattractant receptor interactions is an important question requiring research in the area. Improving CTL recruitment to tumors by enhancing $\mathrm{LTB}_{4}, \mathrm{CXCL9}$ and CXCL10 ligand levels can potentiate the anti-tumor responses. Alternatively, our study has potential implications in improvement of CAR-based ACT approaches. Antigen specific chimeric antigen receptors with BLT1 or CXCR3 could be designed to improve tumor infiltration of the transferred cells and probably reduce toxicities associated with off-target effects of CAR therapies.

It was recently demonstrated by Mikuchi. et.al using intra-vital microscopy that CXCR3 is crucial for enabling adhesion and extravasation of antigen specific $\mathrm{T}$ cells through the tumor endothelium. At what juncture is BLT1 required in this process could be answered.

Future directions of this project could be aimed at understanding the specific cell types involved in BLT1 and CXCR3 production in tumors. We currently believe that myeloid cells including macrophages and mast cells are the major producers of $\mathrm{LTB}_{4}$ at the sites of inflammation. Investigating the same in the context of tumors would be important.

While it is well demonstrated in various studies that immunotherapy including PD-1 blockade strategies significantly induce CXCR3 ligands CXCL9 and 
CXCL10 in the tumors to aid additional effector cells to migrate in response to the increased chemokine gradient in tumors, it would be interesting to understand whether PD-1 blockade therapy in melanoma patients induces $\mathrm{LTB}_{4}$ production in tumor biopsies. This would add a new mechanism in PD-1 blockade mediated chemoattractant induction in the tumor.

We demonstrated that Anti-PD-1 based vaccine completely lost its efficacy in $\mathrm{BLT}^{-/-}$and $\mathrm{CXCR}^{-/-}$mice. The question still remains whether defective $\mathrm{CTL}$ migration is the only cause for the loss in efficacy of the vaccine in knockout mice. Possible future experiments must be directed to understand whether tumor mutation based neo-antigen-specific T cells in WT versus knockout tumors is different. The hypothesis here is that in the absence of CXCR3 and BLT1 receptors, clonal diversity of neo-antigen specific $\mathrm{T}$ cells would be diminished. TCR-repertoire sequencing in tumor versus periphery of WT as well as BLT1 $1^{-1}$ and $\mathrm{CXCR} 3^{-/-}$mice would be essential in answering this question. 


\section{REFERENCES}

1. Kinzler, K.W. and B. Vogelstein, Lessons from hereditary colorectal cancer. Cell, 1996. 87(2): p. 159-70.

2. Hanahan, D. and R.A. Weinberg, The hallmarks of cancer. Cell, 2000. 100(1): p. 57-70.

3. Smyth, M.J., G.P. Dunn, and R.D. Schreiber, Cancer immunosurveillance and immunoediting: the roles of immunity in suppressing tumor development and shaping tumor immunogenicity. Adv Immunol, 2006. 90: p. 1-50.

4. Parish, C.R., Cancer immunotherapy: the past, the present and the future. Immunol Cell Biol, 2003. 81(2): p. 106-13.

5. Rhodes, J.M. and B.J. Campbell, Inflammation and colorectal cancer: IBD-associated and sporadic cancer compared. Trends Mol Med, 2002. 8(1): p. 10-6.

6. Ernst, P.B. and B.D. Gold, The disease spectrum of Helicobacter pylori: the immunopathogenesis of gastroduodenal ulcer and gastric cancer. Annu Rev Microbiol, 2000. 54: p. 615-40.

7. de Oliveria Andrade, L.J., et al., Association between hepatitis $C$ and hepatocellular carcinoma. J Glob Infect Dis, 2009. 1(1): p. 33-7.

8. Rakoff-Nahoum, S., Why cancer and inflammation? Yale J Biol Med, 2006. 79(3-4): p. 123-30.

9. Mantovani, A., et al., Cancer-related inflammation. Nature, 2008. 454(7203): p. 436-44.

10. Greten, F.R., et al., IKKbeta links inflammation and tumorigenesis in a mouse model of colitis-associated cancer. Cell, 2004. 118(3): p. 285-96.

11. Karin, M., NF-kappaB as a critical link between inflammation and cancer. Cold Spring Harb Perspect Biol, 2009. 1(5): p. a000141.

12. Balkwill, F., Cancer and the chemokine network. Nat Rev Cancer, 2004. 4(7): p. 540-50.

13. Shankaran, V., et al., IFNgamma and lymphocytes prevent primary tumour development and shape tumour immunogenicity. Nature, 2001. 410(6832): p. 1107-11.

14. Kaplan, D.H., et al., Demonstration of an interferon gamma-dependent tumor surveillance system in immunocompetent mice. Proc Natl Acad Sci U S A, 1998. 95(13): p. 7556-61.

15. Dunn, G.P., et al., Cancer immunoediting: from immunosurveillance to tumor escape. Nat Immunol, 2002. 3(11): p. 991-8.

16. Mittal, D., et al., New insights into cancer immunoediting and its three component phases--elimination, equilibrium and escape. Curr Opin Immunol, 2014. 27: p. 16-25.

17. Teng, M.W., et al., Opposing roles for IL-23 and IL-12 in maintaining occult cancer in an equilibrium state. Cancer Res, 2012. 72(16): p. 3987-96.

18. Schreiber, R.D., L.J. Old, and M.J. Smyth, Cancer immunoediting: integrating immunity's roles in cancer suppression and promotion. Science, 2011. 331(6024): p. 1565-70.

19. Kalbasi, A., et al., Radiation and immunotherapy: a synergistic combination. J Clin Invest, 2013. 123(7): p. 2756-63. 
20. Gebremeskel, S. and B. Johnston, Concepts and mechanisms underlying chemotherapy induced immunogenic cell death: Impact on clinical studies and considerations for combined therapies. Oncotarget, 2015.

21. Chow, M.T., A. Moller, and M.J. Smyth, Inflammation and immune surveillance in cancer. Semin Cancer Biol, 2012. 22(1): p. 23-32.

22. Deng, L., et al., STING-Dependent Cytosolic DNA Sensing Promotes Radiation-Induced Type I Interferon-Dependent Antitumor Immunity in Immunogenic Tumors. Immunity, 2014. 41(5): p. 843-52.

23. Kroemer, G., et al., Immunogenic cell death in cancer therapy. Annu Rev Immunol, 2013. 31: p. 51-72.

24. Bracci, L., et al., Immune-based mechanisms of cytotoxic chemotherapy: implications for the design of novel and rationale-based combined treatments against cancer. Cell Death Differ, 2014. 21(1): p. 15-25.

25. Ghiringhelli, F., et al., Activation of the NLRP3 inflammasome in dendritic cells induces IL1 beta-dependent adaptive immunity against tumors. Nat Med, 2009. 15(10): p. 1170-8.

26. Apetoh, L., et al., Toll-like receptor 4-dependent contribution of the immune system to anticancer chemotherapy and radiotherapy. Nat Med, 2007. 13(9): p. 1050-9.

27. Littman, D.R., Releasing the Brakes on Cancer Immunotherapy. Cell, 2015. 162(6): p. 1186-90.

28. Gubin, M.M. and R.D. Schreiber, CANCER. The odds of immunotherapy success. Science, 2015. 350(6257): p. 158-9.

29. Atkins, M.B. and M. Sznol, Cancer Immunotherapy: Past Progress and Future Directions. Semin Oncol, 2015. 42(4): p. 518-22.

30. Grimm, E.A., et al., Lymphokine-activated killer cell phenomenon. Lysis of natural killerresistant fresh solid tumor cells by interleukin 2-activated autologous human peripheral blood lymphocytes. J Exp Med, 1982. 155(6): p. 1823-41.

31. Mazumder, A., et al., Lysis of fresh human solid tumors by autologous lymphocytes activated in vitro with lectins. Cancer Res, 1982. 42(3): p. 913-8.

32. Kirkwood, J.M. and M.S. Ernstoff, Interferons in the treatment of human cancer. J Clin Oncol, 1984. 2(4): p. 336-52.

33. Wood, C., A vaccine for renal cancer. Lancet, 2008. 372(9648): p. 1460-1.

34. Kantoff, P.W., et al., Sipuleucel-T immunotherapy for castration-resistant prostate cancer. N Engl J Med, 2010. 363(5): p. 411-22.

35. Chen, L. and X. Han, Anti-PD-1/PD-L1 therapy of human cancer: past, present, and future. J Clin Invest, 2015. 125(9): p. 3384-91.

36. Atkins, M.B., et al., High-dose recombinant interleukin-2 therapy in patients with metastatic melanoma: long-term survival update. Cancer J Sci Am, 2000. 6 Suppl 1: p. S11-4.

37. Fisher, R.I., S.A. Rosenberg, and G. Fyfe, Long-term survival update for high-dose recombinant interleukin-2 in patients with renal cell carcinoma. Cancer J Sci Am, 2000. 6 Suppl 1: p. S55-7.

38. Kirkwood, J.M., et al., High-and low-dose interferon alfa-2b in high-risk melanoma: first analysis of intergroup trial E1690/S9111/C9190. J Clin Oncol, 2000. 18(12): p. 2444-58.

39. Eggermont, A.M., M. Maio, and C. Robert, Immune checkpoint inhibitors in melanoma provide the cornerstones for curative therapies. Semin Oncol, 2015. 42(3): p. 429-35.

40. Sharma, R.K., et al., Regulation of cytotoxic T-Lymphocyte trafficking to tumors by chemoattractants: implications for immunotherapy. Expert Rev Vaccines, 2015. 14(4): p. 537-49. 
41. Mellman, I., G. Coukos, and G. Dranoff, Cancer immunotherapy comes of age. Nature, 2011. 480(7378): p. 480-9.

42. Capece, D., et al., Targeting costimulatory molecules to improve antitumor immunity. J Biomed Biotechnol, 2012. 2012: p. 926321.

43. Jiang, T. and C. Zhou, The past, present and future of immunotherapy against tumor. Transl Lung Cancer Res, 2015. 4(3): p. 253-64.

44. Feldman, S.A., et al., Adoptive Cell Therapy-Tumor-Infiltrating Lymphocytes, T-Cell Receptors, and Chimeric Antigen Receptors. Semin Oncol, 2015. 42(4): p. 626-39.

45. Callahan, M.K. and J.D. Wolchok, At the bedside: CTLA-4- and PD-1-blocking antibodies in cancer immunotherapy. J Leukoc Biol, 2013. 94(1): p. 41-53.

46. Leach, D.R., M.F. Krummel, and J.P. Allison, Enhancement of antitumor immunity by CTLA-4 blockade. Science, 1996. 271(5256): p. 1734-6.

47. Hirano, F., et al., Blockade of B7-H1 and PD-1 by monoclonal antibodies potentiates cancer therapeutic immunity. Cancer Res, 2005. 65(3): p. 1089-96.

48. Ascierto, M.L., I. Melero, and P.A. Ascierto, Melanoma: From Incurable Beast to a Curable Bet. The Success of Immunotherapy. Front Oncol, 2015. 5: p. 152.

49. Hodi, F.S., et al., Improved survival with ipilimumab in patients with metastatic melanoma. N Engl J Med, 2010. 363(8): p. 711-23.

50. Ishida, Y., et al., Induced expression of PD-1, a novel member of the immunoglobulin gene superfamily, upon programmed cell death. EMBO J, 1992. 11(11): p. 3887-95.

51. Barber, D.L., et al., Restoring function in exhausted CD8 T cells during chronic viral infection. Nature, 2006. 439(7077): p. 682-7.

52. Day, C.L., et al., PD-1 expression on HIV-specific T cells is associated with T-cell exhaustion and disease progression. Nature, 2006. 443(7109): p. 350-4.

53. Ahmadzadeh, M., et al., Tumor antigen-specific CD8 T cells infiltrating the tumor express high levels of PD-1 and are functionally impaired. Blood, 2009. 114(8): p. 1537-44.

54. Curran, M.A., et al., PD-1 and CTLA-4 combination blockade expands infiltrating $T$ cells and reduces regulatory $T$ and myeloid cells within B16 melanoma tumors. Proc Natl Acad Sci U S A, 2010. 107(9): p. 4275-80.

55. Rizvi, N.A., et al., Cancer immunology. Mutational landscape determines sensitivity to PD-1 blockade in non-small cell lung cancer. Science, 2015. 348(6230): p. 124-8.

56. Xiao, Y. and G.J. Freeman, The microsatellite instable subset of colorectal cancer is a particularly good candidate for checkpoint blockade immunotherapy. Cancer Discov, 2015. 5(1): p. 16-8.

57. Smyrk, T.C., et al., Tumor-infiltrating lymphocytes are a marker for microsatellite instability in colorectal carcinoma. Cancer, 2001. 91(12): p. 2417-22.

58. Vilar, E. and S.B. Gruber, Microsatellite instability in colorectal cancer-the stable evidence. Nat Rev Clin Oncol, 2010. 7(3): p. 153-62.

59. Llosa, N.J., et al., The vigorous immune microenvironment of microsatellite instable colon cancer is balanced by multiple counter-inhibitory checkpoints. Cancer Discov, 2015. 5(1): p. 43-51.

60. Gattinoni, L., et al., Acquisition of full effector function in vitro paradoxically impairs the in vivo antitumor efficacy of adoptively transferred CD8+ T cells. J Clin Invest, 2005. 115(6): p. 1616-26.

61. Rosenberg, S.A., et al., Adoptive cell transfer: a clinical path to effective cancer immunotherapy. Nat Rev Cancer, 2008. 8(4): p. 299-308.

62. Restifo, N.P., M.E. Dudley, and S.A. Rosenberg, Adoptive immunotherapy for cancer: harnessing the T cell response. Nat Rev Immunol, 2012. 12(4): p. 269-81. 
63. Rosenberg, S.A., et al., Durable complete responses in heavily pretreated patients with metastatic melanoma using T-cell transfer immunotherapy. Clin Cancer Res, 2011. 17(13): p. 4550-7.

64. Robbins, P.F., et al., Tumor regression in patients with metastatic synovial cell sarcoma and melanoma using genetically engineered lymphocytes reactive with NY-ESO-1. J Clin Oncol, 2011. 29(7): p. 917-24.

65. Walia, V., et al., Delving into somatic variation in sporadic melanoma. Pigment Cell Melanoma Res, 2012. 25(2): p. 155-70.

66. Klebanoff, C.A., L. Gattinoni, and N.P. Restifo, Sorting through subsets: which T-cell populations mediate highly effective adoptive immunotherapy? J Immunother, 2012. 35(9): p. 651-60.

67. Yang, S., et al., Modulating the differentiation status of ex vivo-cultured anti-tumor $T$ cells using cytokine cocktails. Cancer Immunol Immunother, 2013. 62(4): p. 727-36.

68. Tran, E., et al., Cancer immunotherapy based on mutation-specific CD4+ $T$ cells in a patient with epithelial cancer. Science, 2014. 344(6184): p. 641-5.

69. Gubin, M.M., et al., Tumor neoantigens: building a framework for personalized cancer immunotherapy. J Clin Invest, 2015. 125(9): p. 3413-21.

70. Gros, A., et al., $P D-1$ identifies the patient-specific CD8(+) tumor-reactive repertoire infiltrating human tumors. J Clin Invest, 2014. 124(5): p. 2246-59.

71. Barrett, D.M., et al., Chimeric antigen receptor therapy for cancer. Annu Rev Med, 2014. 65: p. 333-47.

72. June, C.H., et al., Engineered T cells for cancer therapy. Cancer Immunol Immunother, 2014. 63(9): p. 969-75.

73. Porter, D.L., et al., Chimeric antigen receptor-modified T cells in chronic lymphoid leukemia. N Engl J Med, 2011. 365(8): p. 725-33.

74. Beatty, G.L. and W.L. Gladney, Immune escape mechanisms as a guide for cancer immunotherapy. Clin Cancer Res, 2015. 21(4): p. 687-92.

75. Melero, I., et al., T-cell and NK-cell infiltration into solid tumors: a key limiting factor for efficacious cancer immunotherapy. Cancer Discov, 2014. 4(5): p. 522-6.

76. Rosenberg, S.A., et al., Tumor progression can occur despite the induction of very high levels of self/tumor antigen-specific CD8+ T cells in patients with melanoma. J Immunol, 2005. 175(9): p. 6169-76.

77. Moon, E.K., et al., Expression of a functional CCR2 receptor enhances tumor localization and tumor eradication by retargeted human $T$ cells expressing a mesothelin-specific chimeric antibody receptor. Clin Cancer Res, 2011. 17(14): p. 4719-30.

78. Foxman, E.F., J.J. Campbell, and E.C. Butcher, Multistep navigation and the combinatorial control of leukocyte chemotaxis. J Cell Biol, 1997. 139(5): p. 1349-60.

79. Fernandez, H.N., et al., Chemotactic response to human C3a and C5a anaphylatoxins. I. Evaluation of $\mathrm{C} 3 \mathrm{a}$ and $\mathrm{C} 5$ a leukotaxis in vitro and under stimulated in vivo conditions. J Immunol, 1978. 120(1): p. 109-15.

80. Zlotnik, A. and O. Yoshie, The chemokine superfamily revisited. Immunity, 2012. 36(5): p. 705-16.

81. Chow, M.T. and A.D. Luster, Chemokines in cancer. Cancer Immunol Res, 2014. 2(12): p. 1125-31.

82. Mantovani, A., et al., Chemokines and chemokine receptors during activation and deactivation of monocytes and dendritic cells and in amplification of Th1 versus Th2 responses. Int J Clin Lab Res, 1998. 28(2): p. 77-82. 
83. Mantovani, A., The chemokine system: redundancy for robust outputs. Immunol Today, 1999. 20(6): p. 254-7.

84. Griffith, J.W., C.L. Sokol, and A.D. Luster, Chemokines and chemokine receptors: positioning cells for host defense and immunity. Annu Rev Immunol, 2014. 32: p. 659702.

85. Lazennec, G. and A. Richmond, Chemokines and chemokine receptors: new insights into cancer-related inflammation. Trends Mol Med, 2010. 16(3): p. 133-44.

86. Payne, A.S. and L.A. Cornelius, The role of chemokines in melanoma tumor growth and metastasis. J Invest Dermatol, 2002. 118(6): p. 915-22.

87. Smith, M.C., et al., CXCR4 regulates growth of both primary and metastatic breast cancer. Cancer Res, 2004. 64(23): p. 8604-12.

88. Luker, K.E. and G.D. Luker, Functions of CXCL12 and CXCR4 in breast cancer. Cancer Lett, 2006. 238(1): p. 30-41.

89. Groom, J.R., et al., CXCR3 chemokine receptor-ligand interactions in the lymph node optimize CD4+ T helper 1 cell differentiation. Immunity, 2012. 37(6): p. 1091-103.

90. Groom, J.R. and A.D. Luster, CXCR3 in T cell function. Exp Cell Res, 2011. 317(5): p. 62031.

91. Vesely, M.D., et al., Natural innate and adaptive immunity to cancer. Annu Rev Immunol, 2011. 29: p. 235-71.

92. Oghumu, S., et al., CXCR3 deficiency enhances tumor progression by promoting macrophage M2 polarization in a murine breast cancer model. Immunology, 2014. 143(1): p. 109-19.

93. Gonzalez-Martin, A., et al., Maximal T cell-mediated antitumor responses rely upon CCR5 expression in both CD4(+) and CD8(+) T cells. Cancer Res, 2011. 71(16): p. 5455-66.

94. Josefowicz, S.Z., L.F. Lu, and A.Y. Rudensky, Regulatory T cells: mechanisms of differentiation and function. Annu Rev Immunol, 2012. 30: p. 531-64.

95. Curiel, T.J., et al., Specific recruitment of regulatory T cells in ovarian carcinoma fosters immune privilege and predicts reduced survival. Nat Med, 2004. 10(9): p. 942-9.

96. Olkhanud, P.B., et al., Breast cancer lung metastasis requires expression of chemokine receptor CCR4 and regulatory T cells. Cancer Res, 2009. 69(14): p. 5996-6004.

97. Schlecker, E., et al., Tumor-infiltrating monocytic myeloid-derived suppressor cells mediate CCR5-dependent recruitment of regulatory T cells favoring tumor growth. J Immunol, 2012. 189(12): p. 5602-11.

98. Facciabene, A., et al., Tumour hypoxia promotes tolerance and angiogenesis via CCL28 and $T$ (reg) cells. Nature, 2011. 475(7355): p. 226-30.

99. Redjimi, N., et al., CXCR3 + T regulatory cells selectively accumulate in human ovarian carcinomas to limit type I immunity. Cancer Res, 2012. 72(17): p. 4351-60.

100. Tager, A.M., et al., Leukotriene B4 receptor BLT1 mediates early effector $T$ cell recruitment. Nat Immunol, 2003. 4(10): p. 982-90.

101. Viola, A. and A.D. Luster, Chemokines and their receptors: drug targets in immunity and inflammation. Annu Rev Pharmacol Toxicol, 2008. 48: p. 171-97.

102. Freeman, C.M., J.L. Curtis, and S.W. Chensue, CC chemokine receptor 5 and CXC chemokine receptor 6 expression by lung CD8+ cells correlates with chronic obstructive pulmonary disease severity. Am J Pathol, 2007. 171(3): p. 767-76.

103. Fyhrquist, N., et al., CD8+ T cell migration to the skin requires $C D 4+$ help in a murine model of contact hypersensitivity. PLoS One, 2012. 7(8): p. e41038. 
104. Perez de Lema, G., et al., Chemokine receptor Ccr2 deficiency reduces renal disease and prolongs survival in MRL/Ipr lupus-prone mice. J Am Soc Nephrol, 2005. 16(12): p. 3592601.

105. Nanki, T., et al., Migration of CX3CR1-positive T cells producing type 1 cytokines and cytotoxic molecules into the synovium of patients with rheumatoid arthritis. Arthritis Rheum, 2002. 46(11): p. 2878-83.

106. Gunther, C., et al., CXCL16 and CXCR6 are upregulated in psoriasis and mediate cutaneous recruitment of human CD8+ T cells. J Invest Dermatol, 2012. 132(3 Pt 1): p. 626-34.

107. Friese, M.A. and L. Fugger, Autoreactive CD8+ T cells in multiple sclerosis: a new target for therapy? Brain, 2005. 128(Pt 8): p. 1747-63.

108. Helbig, K.J., et al., Differential expression of the CXCR3 ligands in chronic hepatitis $C$ virus (HCV) infection and their modulation by HCV in vitro. J Virol, 2009. 83(2): p. 836-46.

109. Nakanishi, Y., et al., CD8(+) T lymphocyte mobilization to virus-infected tissue requires CD4(+) T-cell help. Nature, 2009. 462(7272): p. 510-3.

110. Harris, T.H., et al., Generalized Levy walks and the role of chemokines in migration of effector CD8+ T cells. Nature, 2012. 486(7404): p. 545-8.

111. Abdi, R., T.K. Means, and A.D. Luster, Chemokines in islet allograft rejection. Diabetes Metab Res Rev, 2003. 19(3): p. 186-90.

112. Akashi, S., et al., A novel small-molecule compound targeting CCR5 and CXCR3 prevents acute and chronic allograft rejection. Transplantation, 2005. 80(3): p. 378-84.

113. Sato, T., et al., Role for CXCR6 in recruitment of activated CD8+ lymphocytes to inflamed liver. J Immunol, 2005. 174(1): p. 277-83.

114. Mrass, P., et al., Random migration precedes stable target cell interactions of tumorinfiltrating T cells. J Exp Med, 2006. 203(12): p. 2749-61.

115. Mrass, P., et al., CD44 mediates successful interstitial navigation by killer $T$ cells and enables efficient antitumor immunity. Immunity, 2008. 29(6): p. 971-85.

116. Boissonnas, A., et al., In vivo imaging of cytotoxic $T$ cell infiltration and elimination of a solid tumor. J Exp Med, 2007. 204(2): p. 345-56.

117. Deguine, J., et al., Intravital imaging reveals distinct dynamics for natural killer and CD8(+) T cells during tumor regression. Immunity, 2010. 33(4): p. 632-44.

118. Salmon, H., et al., Matrix architecture defines the preferential localization and migration of T cells into the stroma of human lung tumors. J Clin Invest, 2012. 122(3): p. 899-910.

119. Bindea, G., et al., Spatiotemporal dynamics of intratumoral immune cells reveal the immune landscape in human cancer. Immunity, 2013. 39(4): p. 782-95.

120. Ohta, M., et al., The high expression of Fractalkine results in a better prognosis for colorectal cancer patients. Int J Oncol, 2005. 26(1): p. 41-7.

121. Tang, L., et al., Gene therapy with CX3CL1/Fractalkine induces antitumor immunity to regress effectively mouse hepatocellular carcinoma. Gene Ther, 2007. 14(16): p. 122634.

122. Xin, H., et al., Antitumor immune response by CX3CL1 fractalkine gene transfer depends on both NK and T cells. Eur J Immunol, 2005. 35(5): p. 1371-80.

123. Zeng, Y., et al., Fractalkine (CX3CL1)- and interleukin-2-enriched neuroblastoma microenvironment induces eradication of metastases mediated by $T$ cells and natural killer cells. Cancer Res, 2007. 67(5): p. 2331-8.

124. Guo, J., et al., Fractalkine transgene induces T-cell-dependent antitumor immunity through chemoattraction and activation of dendritic cells. Int J Cancer, 2003. 103(2): p. 212-20. 
125. Matsumura, S., et al., Radiation-induced CXCL16 release by breast cancer cells attracts effector T cells. J Immunol, 2008. 181(5): p. 3099-107.

126. Draghiciu, O., et al., Therapeutic immunization and local low-dose tumor irradiation, a reinforcing combination. Int J Cancer, 2014. 134(4): p. 859-72.

127. Lavergne, E., et al., Intratumoral CC chemokine ligand 5 overexpression delays tumor growth and increases tumor cell infiltration. J Immunol, 2004. 173(6): p. 3755-62.

128. Franciszkiewicz, K., et al., Intratumoral induction of CD103 triggers tumor-specific CTL function and CCR5-dependent T-cell retention. Cancer Res, 2009. 69(15): p. 6249-55.

129. Gough, M., et al., Gene therapy to manipulate effector T cell trafficking to tumors for immunotherapy. J Immunol, 2005. 174(9): p. 5766-73.

130. Moran, C.J., et al., RANTES expression is a predictor of survival in stage I lung adenocarcinoma. Clin Cancer Res, 2002. 8(12): p. 3803-12.

131. Niwa, Y., et al., Correlation of tissue and plasma RANTES levels with disease course in patients with breast or cervical cancer. Clin Cancer Res, 2001. 7(2): p. 285-9.

132. Chang, L.Y., et al., Tumor-derived chemokine CCL5 enhances TGF-beta-mediated killing of CD8(+) T cells in colon cancer by T-regulatory cells. Cancer Res, 2012. 72(5): p. 1092-102.

133. Hancock, W.W., et al., Requirement of the chemokine receptor CXCR3 for acute allograft rejection. J Exp Med, 2000. 192(10): p. 1515-20.

134. Cheong, H.S., et al., CXCR3 polymorphisms associated with risk of asthma. Biochem Biophys Res Commun, 2005. 334(4): p. 1219-25.

135. Im, C.H., et al., CXCR3 polymorphism is associated with male gender and pleuritis in patients with systemic lupus erythematosus. Hum Immunol, 2014. 75(5): p. 466-9.

136. Huang, H., Y. Liu, and J. Xiang, Synergistic effect of adoptive T-cell therapy and intratumoral interferon gamma-inducible protein-10 transgene expression in treatment of established tumors. Cell Immunol, 2002. 217(1-2): p. 12-22.

137. Mullins, I.M., et al., CXC chemokine receptor 3 expression by activated CD $8+T$ cells is associated with survival in melanoma patients with stage III disease. Cancer Res, 2004. 64(21): p. 7697-701.

138. Dengel, L.T., et al., Interferons induce CXCR3-cognate chemokine production by human metastatic melanoma. J Immunother, 2010. 33(9): p. 965-74.

139. Musha, H., et al., Selective infiltration of CCR5(+)CXCR3(+) T lymphocytes in human colorectal carcinoma. Int J Cancer, 2005. 116(6): p. 949-56.

140. Liu, Y., et al., Chemokine receptors support infiltration of lymphocyte subpopulations in human hepatocellular carcinoma. Clin Immunol, 2005. 114(2): p. 174-82.

141. Liu, Y.Q., et al., Desensitization of T lymphocyte function by CXCR3 ligands in human hepatocellular carcinoma. World J Gastroenterol, 2005. 11(2): p. 164-70.

142. Mikucki, M.E., et al., Non-redundant requirement for CXCR3 signalling during tumoricidal T-cell trafficking across tumour vascular checkpoints. Nat Commun, 2015. 6: p. 7458.

143. Clancy-Thompson, E., et al., Melanoma Induces, and Adenosine Suppresses, CXCR3Cognate Chemokine Production and T-cell Infiltration of Lungs Bearing Metastatic-like Disease. Cancer Immunol Res, 2015. 3(8): p. 956-67.

144. Franciszkiewicz, K., et al., Role of chemokines and chemokine receptors in shaping the effector phase of the antitumor immune response. Cancer Res, 2012. 72(24): p. 6325-32.

145. Fisher, D.T., et al., Hurdles to lymphocyte trafficking in the tumor microenvironment: implications for effective immunotherapy. Immunol Invest, 2006. 35(3-4): p. 251-77.

146. Bellone, M. and A. Calcinotto, Ways to enhance lymphocyte trafficking into tumors and fitness of tumor infiltrating lymphocytes. Front Oncol, 2013. 3: p. 231. 
147. Maric, M. and Y. Liu, Strong cytotoxic T lymphocyte responses to a macrophage inflammatory protein 1alpha-expressing tumor: linkage between inflammation and specific immunity. Cancer Res, 1999. 59(21): p. 5549-53.

148. Mule, J.J., et al., RANTES secretion by gene-modified tumor cells results in loss of tumorigenicity in vivo: role of immune cell subpopulations. Hum Gene Ther, 1996. 7(13): p. 1545-53.

149. Fushimi, T., et al., Macrophage inflammatory protein 3alpha transgene attracts dendritic cells to established murine tumors and suppresses tumor growth. J Clin Invest, 2000. 105(10): p. 1383-93.

150. Orinska, Z., et al., TLR3-induced activation of mast cells modulates CD8+ T-cell recruitment. Blood, 2005. 106(3): p. 978-87.

151. Uekusa, Y., et al., A pivotal role for CC chemokine receptor 5 in T-cell migration to tumor sites induced by interleukin 12 treatment in tumor-bearing mice. Cancer Res, 2002. 62(13): p. 3751-8.

152. Golden, E.B., et al., An abscopal response to radiation and ipilimumab in a patient with metastatic non-small cell lung cancer. Cancer Immunol Res, 2013. 1(6): p. 365-72.

153. Lutsiak, M.E., et al., Inhibition of CD4(+)25+ Tregulatory cell function implicated in enhanced immune response by low-dose cyclophosphamide. Blood, 2005. 105(7): p. 2862-8.

154. Suzuki, E., et al., Gemcitabine selectively eliminates splenic Gr-1+/CD11b+ myeloid suppressor cells in tumor-bearing animals and enhances antitumor immune activity. Clin Cancer Res, 2005. 11(18): p. 6713-21.

155. Lim, J.Y., et al., Type I interferons induced by radiation therapy mediate recruitment and effector function of CD8(+) T cells. Cancer Immunol Immunother, 2014. 63(3): p. 259-71.

156. Di Stasi, A., et al., T lymphocytes coexpressing CCR4 and a chimeric antigen receptor targeting CD30 have improved homing and antitumor activity in a Hodgkin tumor model. Blood, 2009. 113(25): p. 6392-402.

157. $\mathrm{Xu}, \mathrm{Y}$., et al., Optogenetic control of chemokine receptor signal and T-cell migration. Proc Natl Acad Sci U S A, 2014. 111(17): p. 6371-6.

158. Koebel, C.M., et al., Adaptive immunity maintains occult cancer in an equilibrium state. Nature, 2007. 450(7171): p. 903-7.

159. Wilcox, R.A., et al., Impaired infiltration of tumor-specific cytolytic $T$ cells in the absence of interferon-gamma despite their normal maturation in lymphoid organs during CD137 monoclonal antibody therapy. Cancer Res, 2002. 62(15): p. 4413-8.

160. Engelhardt, J.J., et al., Marginating dendritic cells of the tumor microenvironment crosspresent tumor antigens and stably engage tumor-specific T cells. Cancer Cell, 2012. 21(3): p. 402-17.

161. Boissonnas, A., et al., CD8+ tumor-infiltrating T cells are trapped in the tumor-dendritic cell network. Neoplasia, 2013. 15(1): p. 85-94.

162. Harlin, H., et al., Chemokine expression in melanoma metastases associated with CD8+ T-cell recruitment. Cancer Res, 2009. 69(7): p. 3077-85.

163. Molon, B., et al., Chemokine nitration prevents intratumoral infiltration of antigenspecific T cells. J Exp Med, 2011. 208(10): p. 1949-62.

164. Pivarcsi, A., et al., Tumor immune escape by the loss of homeostatic chemokine expression. Proc Natl Acad Sci U S A, 2007. 104(48): p. 19055-60.

165. Proost, P., et al., Proteolytic processing of CXCL11 by CD13/aminopeptidase N impairs CXCR3 and CXCR7 binding and signaling and reduces lymphocyte and endothelial cell migration. Blood, 2007. 110(1): p. 37-44. 
166. Motz, G.T. and G. Coukos, The parallel lives of angiogenesis and immunosuppression: cancer and other tales. Nat Rev Immunol, 2011. 11(10): p. 702-11.

167. Zhang, L., et al., Intratumoral T cells, recurrence, and survival in epithelial ovarian cancer. N Engl J Med, 2003. 348(3): p. 203-13.

168. Chung, A.S., J. Lee, and N. Ferrara, Targeting the tumour vasculature: insights from physiological angiogenesis. Nat Rev Cancer, 2010. 10(7): p. 505-14.

169. Back, M., et al., Update on leukotriene, lipoxin and oxoeicosanoid receptors: IUPHAR Review 7. Br J Pharmacol, 2014. 171(15): p. 3551-74.

170. Samuelsson, B., et al., Leukotrienes and lipoxins: structures, biosynthesis, and biological effects. Science, 1987. 237(4819): p. 1171-6.

171. Matsumoto, T., et al., Molecular cloning and amino acid sequence of human 5lipoxygenase. Proc Natl Acad Sci U S A, 1988. 85(1): p. 26-30.

172. Minami, M., et al., Molecular cloning of a cDNA coding for human leukotriene A4 hydrolase. Complete primary structure of an enzyme involved in eicosanoid synthesis. J Biol Chem, 1987. 262(29): p. 13873-6.

173. Sala, A., et al., Release of leukotriene A4 versus leukotriene B4 from human polymorphonuclear leukocytes. J Biol Chem, 1996. 271(30): p. 17944-8.

174. Yokomizo, T., et al., A G-protein-coupled receptor for leukotriene B4 that mediates chemotaxis. Nature, 1997. 387(6633): p. 620-4.

175. Yokomizo, T., et al., A second leukotriene B(4) receptor, BLT2. A new therapeutic target in inflammation and immunological disorders. J Exp Med, 2000. 192(3): p. 421-32.

176. Sharma, R.K., et al., Expression of leukotriene B(4) receptor-1 on CD8(+) T cells is required for their migration into tumors to elicit effective antitumor immunity. J Immunol, 2013. 191(6): p. 3462-70.

177. Jala, V.R. and B. Haribabu, Leukotrienes and atherosclerosis: new roles for old mediators. Trends Immunol, 2004. 25(6): p. 315-22.

178. Tager, A.M. and A.D. Luster, BLT1 and BLT2: the leukotriene B(4) receptors. Prostaglandins Leukot Essent Fatty Acids, 2003. 69(2-3): p. 123-34.

179. Haribabu, B., et al., Targeted disruption of the leukotriene $B(4)$ receptor in mice reveals its role in inflammation and platelet-activating factor-induced anaphylaxis. J Exp Med, 2000. 192(3): p. 433-8.

180. Kim, N.D., et al., A unique requirement for the leukotriene B4 receptor BLT1 for neutrophil recruitment in inflammatory arthritis. J Exp Med, 2006. 203(4): p. 829-35.

181. Li, R.C., et al., Leukotriene B4 receptor-1 mediates intermittent hypoxia-induced atherogenesis. Am J Respir Crit Care Med, 2011. 184(1): p. 124-31.

182. Miyahara, N., et al., Leukotriene B4 receptor 1 expression on dendritic cells is required for the development of Th2 responses and allergen-induced airway hyperresponsiveness. J Immunol, 2008. 181(2): p. 1170-8.

183. Liao, T., et al., Blockade of the interaction of leukotriene $b 4$ with its receptor prevents development of autoimmune uveitis. Invest Ophthalmol Vis Sci, 2006. 47(4): p. 1543-9.

184. Subbarao, K., et al., Role of leukotriene B4 receptors in the development of atherosclerosis: potential mechanisms. Arterioscler Thromb Vasc Biol, 2004. 24(2): p. 369-75.

185. Fan, Z., et al., NK-cell activation by LIGHT triggers tumor-specific CD8+ T-cell immunity to reject established tumors. Blood, 2006. 107(4): p. 1342-51.

186. Satpathy, S.R., et al., Crystalline silica-induced leukotriene B4-dependent inflammation promotes lung tumour growth. Nat Commun, 2015. 6: p. 7064. 
187. Yokota, Y., et al., Absence of LTB4/BLT1 axis facilitates generation of mouse GM-CSFinduced long-lasting antitumor immunologic memory by enhancing innate and adaptive immune systems. Blood, 2012. 120(17): p. 3444-54.

188. Molon, B., et al., T cell costimulation by chemokine receptors. Nat Immunol, 2005. 6(5): p. 465-71.

189. Ohnishi, H., et al., Corticosteroids enhance CD8+ T cell-mediated airway hyperresponsiveness and allergic inflammation by upregulating leukotriene $B 4$ receptor 1. J Allergy Clin Immunol, 2008. 121(4): p. 864-71 e4.

190. Mathis, S.P., et al., Nonredundant roles for leukotriene B4 receptors BLT1 and BLT2 in inflammatory arthritis. J Immunol, 2010. 185(5): p. 3049-56.

191. Spite, M., et al., Deficiency of the leukotriene B4 receptor, BLT-1, protects against systemic insulin resistance in diet-induced obesity. J Immunol, 2011. 187(4): p. 1942-9.

192. Chou, R.C., et al., Lipid-cytokine-chemokine cascade drives neutrophil recruitment in a murine model of inflammatory arthritis. Immunity, 2010. 33(2): p. 266-78.

193. Overwijk, W.W. and N.P. Restifo, B16 as a mouse model for human melanoma. Curr Protoc Immunol, 2001. Chapter 20: p. Unit 201.

194. Bachi, A.L., et al., Leukotriene B4 creates a favorable microenvironment for murine melanoma growth. Mol Cancer Res, 2009. 7(9): p. 1417-24.

195. Wejksza, K., et al., Cancer-produced metabolites of 5-lipoxygenase induce tumor-evoked regulatory $B$ cells via peroxisome proliferator-activated receptor alpha. J Immunol, 2013. 190(6): p. 2575-84.

196. Tong, W.G., et al., Leukotriene B4 receptor antagonist LY293111 inhibits proliferation and induces apoptosis in human pancreatic cancer cells. Clin Cancer Res, 2002. 8(10): p. 3232-42.

197. Ihara, A., et al., Blockade of leukotriene B4 signaling pathway induces apoptosis and suppresses cell proliferation in colon cancer. J Pharmacol Sci, 2007. 103(1): p. 24-32.

198. Shi, Y., et al., Granulocyte-macrophage colony-stimulating factor (GM-CSF) and T-cell responses: what we do and don't know. Cell Res, 2006. 16(2): p. 126-33.

199. Fukushima, A., et al., Genetic background determines susceptibility to experimental immune-mediated blepharoconjunctivitis: comparison of Balb/c and C57BL/6 mice. Exp Eye Res, 2006. 82(2): p. 210-8.

200. Kitajima, M., et al., Memory type 2 helper T cells induce long-lasting antitumor immunity by activating natural killer cells. Cancer Res, 2011. 71(14): p. 4790-8.

201. Hong, M., et al., Chemotherapy induces intratumoral expression of chemokines in cutaneous melanoma, favoring T-cell infiltration and tumor control. Cancer Res, 2011. 71(22): p. 6997-7009.

202. Sung, J.H., et al., Chemokine guidance of central memory T cells is critical for antiviral recall responses in lymph nodes. Cell, 2012. 150(6): p. 1249-63.

203. Liu, L., et al., Severe disease, unaltered leukocyte migration, and reduced IFN-gamma production in CXCR3-/- mice with experimental autoimmune encephalomyelitis. J Immunol, 2006. 176(7): p. 4399-409.

204. Campbell, J.D., et al., Allergic humans are hyporesponsive to a CXCR3 ligand-mediated Th1 immunity-promoting loop. FASEB J, 2004. 18(2): p. 329-31.

205. Ostrand-Rosenberg, S., CD4+ Tlymphocytes: a critical component of antitumor immunity. Cancer Invest, 2005. 23(5): p. 413-9.

206. Waldhauer, I. and A. Steinle, NK cells and cancer immunosurveillance. Oncogene, 2008. 27(45): p. 5932-43. 
207. Cekic, C., et al., Adenosine A2B receptor blockade slows growth of bladder and breast tumors. J Immunol, 2012. 188(1): p. 198-205.

208. Ostrand-Rosenberg, S., L.A. Horn, and S.T. Haile, The programmed death-1 immunesuppressive pathway: barrier to antitumor immunity. J Immunol, 2014. 193(8): p. 383541.

209. Sznol, M. and L. Chen, Antagonist antibodies to PD-1 and B7-H1 (PD-L1) in the treatment of advanced human cancer. Clin Cancer Res, 2013. 19(5): p. 1021-34.

210. Sakuishi, K., et al., Targeting Tim-3 and PD-1 pathways to reverse $T$ cell exhaustion and restore anti-tumor immunity. J Exp Med, 2010. 207(10): p. 2187-94.

211. Peng, W., et al., PD-1 blockade enhances T-cell migration to tumors by elevating IFNgamma inducible chemokines. Cancer Res, 2012. 72(20): p. 5209-18.

212. Bedognetti, D., et al., CXCR3/CCR5 pathways in metastatic melanoma patients treated with adoptive therapy and interleukin-2. Br J Cancer, 2013. 109(9): p. 2412-23. 


\section{APPENDIX}

\section{LIST OF ABBREVIATIONS}

\section{ABBREVIATION}

1. ACT

2. AOM-DSS

3. CAR

4. CTL

5. CTLA4

6. $\mathrm{COX} 2$

7. DC

8. DKO

9. $\mathrm{ECM}$

10. IL

11. IFN
STANDS FOR

Adoptive cell therapy

Azoxymethane - Dextran sulfate sodium

Chimeric Antigen Receptor

Cytotoxic T lymphocyte or $\mathrm{CD}^{+} \mathrm{T}$ cells

Cytotoxic T lymphocyte associate protein 4

Cyclooxygenase 2

Dendritic Cells

Double Knockout mice

Extra cellular matrix

Interleukin

Interferon 
12. IFNY

13. IDO

14. $\mathrm{LTB}_{4}$

15. MDSC

16. $\mathrm{MHC}$

17. MSI

18. NSAIDs

19. NK

20. NF-K $\beta$

21.PD-1

22. PD-L1

23. PD-L2

24. Rag2

25. ROS

26. RNI

27. STAT

28. TAM
Interferon gamma

Indoleamine-2,3-dioxygenase

Leukotriene $B_{4}$

Myeloid Derived Suppressor Cells

Major histocompatibility complex

Microsatellite Instable

Non-steroidal anti-inflammatory drugs

Natural Killer

Nuclear Factor kappa beta

Programmed cell Death - 1

Programmed cell Death Ligand - 1

Programmed cell Death Ligand - 2

Recombination activating gene 2

Reactive oxygen species

Reactive nitrogen intermediates

Signal Transducer and Activator of transcription

Tumor Associated Macrophages 
29. TdLN

30. Trp2

31. TIL

32. TNFa

33. Treg

34. Th

35. WT
Tumor draining Lymph Node

Tyrosinase related protein-2

Tumor infiltrating leukocytes

Tumor Necrosis Factor alpha

Regulatory T cells

T-helper

Wild Type 


\section{CURRICULUM VITAE \\ ZINAL S. CHHEDA}

Current Address: 833, E. Madison St., Apt \#F, Louisville, KY- 40204.

Tel: $502-554-3762$

Email id: zinal.bt@gmail.com

\section{EDUCATION:}

- 2010-2015 - Ph.D. in Microbiology and Immunology - University of Louisville, USA.

- 2010-2012 - MS in Microbiology and Immunology, University of Louisville, USA.

- 2007-2009 - MS in Biotechnology - University of Mumbai, India.

- 2004-2007 - BS in Biotechnology - University of Mumbai, India.

\section{CURRENT RESEARCH:}

Dissertation Research (2010-2015): "Regulation of CD8 ${ }^{+} \mathrm{T}$ cell trafficking to melanoma tumors by chemo-attractant receptors BLT1 and CXCR3". Mentor: Dr. Haribabu Bodduluri at University of Louisville. 


\section{PAST RESEARCH EXPERIENCE:}

Project 1: "Role of IL-12 - IFNy axis in patients with Mendelian Susceptibility to Mycobacterial Diseases (MSMD)"

Mentor: Dr. Manisha Madkaikar

Place: National Institute of Immunohematology (NIIH), ICMR (2009-2010).

Project 2 (as a partial completion of MS Degree): "The role of $14 \mathrm{bp}$ del/ins polymorphism in exon 8 of the HLA-G gene in Recurrent Spontaneous Abortion (RSA) patients"

Mentor: Late Dr. U. Shankarkumar

Place: National Institute of Immunohematology (NIIH), ICMR (2008-2009).

\section{FELLOWSHIPS:}

1. Careers in Immunology Fellowship by American Association of Immunologists 2014-2015.

2. Integrated Program in Biomedical Sciences Fellowship - 2010-2012.

\section{PROFESSIONAL MEMBERSHIPS:}

American Association of Immunologists (AAI) - 2014 and 201

\section{HONORS AND AWARDS:}

1. AAI Trainee Abstract Award - AAI 2015.

2. The Excellence in Research Award - Department of Microbiology and Immunology at the University of Louisville - 2015. 
3. Graduate student research award $-2^{\text {nd }}$ place at James Graham Brown Cancer Centre Retreat, 2013.

\section{PEER REVIEWED PUBLICATIONS:}

1. Sharma R.K., Chheda Z, Jala VR, Haribabu B. Regulation of cytotoxic TLymphocyte trafficking to tumors by chemoattractants: implications for immunotherapy. Expert Rev Vaccines, 2014: p. 1-13. http://www.ncbi.nlm.nih.gov/pubmed/25482400

2. Sharma RK, Chheda Z, Jala VR, Haribabu B. Expression of leukotriene $\mathrm{B}_{4}$ receptor-1 on $\mathrm{CD}^{+} \mathrm{T}$ cells is required for their migration into tumors to elicit effective antitumor immunity. J. Immunol. 2013.191(6): 3462-70.

http://www.ncbi.nlm.nih.gov/pubmed/23960231

3. U. Shankarkumar, A. Shankarkumar, Z. Chedda, K. Ghosh. Role of 14-bp deletion/insertion polymorphism in exon 8 of the HLA-G gene in recurrent spontaneous abortion patients.

J Hum Reprod Sci. 2011, 4: 143 - 6.

http://www.ncbi.nlm.nih.gov/pubmed/?term=Chedda+Z

\section{PUBLICATIONS IN REVIEW:}

1. Chheda Z., Sharma RK, Jala VR, Haribabu B. Regulation of $\mathrm{CD}^{+} \mathrm{T}$ cell trafficking to tumors and anti-tumor immunity by chemo-attractant receptors BLT1 and CXCR3 - Submitted in Journal of Immunology.

2. Sharma RK, Chheda Z., et.al. A Spontaneous Metastasis Model Reveals the 
significance of Claudin-9 Overexpression in Lung Cancer Metastasis Submitted in International Journal of Cancer.

\section{MANUSCRIPTS UNDER PREPARATION:}

1. Jala VR, Chheda Z., et. al. Ablation of CCR2 reduces the spontaneous intestinal tumorigenesis in $\mathrm{Apc}^{\mathrm{Min} /+}$ mouse model.

2. Sharma RK, Chheda Z., et. al. Interactions within the tumor microenvironment imprints metastatic traits in cancer cells.

3. Jala VR, Chheda Z., et. al. Targeted delivery of UroA to inflamed intestines attenuates inflammatory responses in experimental model of colitis.

\section{ORAL PRESENTATIONS IN BLOCK SYMPOSIUMS:}

1. Chheda Z, Sharma RK, Jala VR, Haribabu B. Regulation of CTL trafficking into tumors and anti-tumor immunity by chemo-attractant receptors BLT1 and CXCR3. American Association of Immunologists (AAl) annual meeting, New Orleans, 2015.

\section{PEER REVIEWED ABSTRACT PUBLICATIONS:}

1. Chheda Z, Sharma RK, Jala VR, Haribabu B. Regulation of CTL trafficking into tumors and anti-tumor immunity by chemo-attractant receptors BLT1 and CXCR3 (TUM7P.1015). J Immunol 2015 194:142.4 
2. Sharma RK, Chheda Z, Jala VR, Haribabu B. Interactions within the tumor microenvironment imprint metastatic traits in cancer cells (TUM6P.1001). $J$ Immunol 2015 194:141.25

3. Sharma RK, Chheda Z, Jala VR, Satpathy SM, Haribabu B. Role and association of $\mathrm{CD} 11 \mathrm{~b}$ and $\mathrm{Gr} 1^{+}$cells in promotion of spontaneous lung cancer metastasis (TUM4P.920) J Immunol 2014 192:138.21

4. Sharma RK, Chheda Z, Jala VR, Haribabu B. Expression of Leukotriene B4 receptor 1 on $\mathrm{CD}^{+} \mathrm{T}$ cells is essential for their migration to tumors, immunesurveillance and cancer immunity (P5106) J Immunol 2013 190:58.7

5. Sharma RK, Chheda Z, Jala VR, Haribabu B. Leukotriene B4 receptor BLT1 expression on $\mathrm{CD}^{+} \mathrm{T}$ cells is critical for antitumor immunity $\mathrm{J}$ Immunol 2012 188:161.5

\section{POSTER PRESENTATION:}

1. Chheda Z., Sharma R.K, V.R. Jala, B. Haribabu. Regulation of CTL trafficking into tumors and anti-tumor immunity by chemo-attractant receptors BLT1 and CXCR3. American Association of Immunologists (AAl), New Orleans, May 10th, 2015.

2. Chheda Z, Sharma R.K, V.R. Jala, B. Haribabu. CTL trafficking into tumors and anti-tumor immunity are finely regulated by the chemokine receptors BLT1 and CXCR3. James Graham Brown Cancer Centre Retreat, 2014. 
3. Sharma R.K., V.R. Jala, Chheda Z, B. Haribabu. Interactions within the tumor microenvironment imprint metastatic traits in cancer cells. James Graham Brown Cancer Centre Retreat, 2014.

4. Chheda Z, Sharma R.K, V.R. Jala, B. Haribabu. CTL trafficking into tumors and anti-tumor immunity are finely regulated by the chemokine receptors BLT1 and CXCR3. James Graham Brown Cancer Centre Retreat, 2013. Graduate Student Research Award - $2^{\text {nd }}$ place.

5. Sharma R.K., V.R. Jala, Chheda Z, B. Haribabu. Enhanced Claudin-9 expression is associated with lung cancer metastasis. James Graham Brown Cancer Centre Retreat, 2011.

6. Chheda Z, U. Shankarkumar, A.Pawar, K. Ghosh. The Role of $14 \mathrm{bp}$ del/ins polymorphism in HLA-G gene in Recurrent Spontaneous Abortion (RSA) patients. Australasian and South East Asian Tissue Typing Association, India, 2009.

7. Chheda Z, U. Shankarkumar, A.Pawar, K. Ghosh. The Role of $14 \mathrm{bp}$ del/ins polymorphism in HLA-G gene in Recurrent Spontaneous Abortion (RSA) patients. 32 $2^{\text {nd }}$ Annual Conference of Mumbai Hematology Group, India, 2009.

8. R. Mitra, Chheda Z, A. Pawar, U. Shankarkumar, K. Ghosh. CCR5 Chemokine receptor delta 32 deletion polymorphism in HIV-1 positive patients. International conference on Fundamental and Translational Research on HIV/AIDS: Global Perspectives, 2009. 